\title{
DETERMINANTS OF PSEUDODIFFERENTIAL OPERATORS AND COMPLEX DEFORMATIONS OF PHASE SPACE*
}

\author{
A. MELIN ${ }^{\dagger}$ AND J. SJÖSTRAND $\ddagger$
}

\begin{abstract}
Consider an h-pseudodifferential operator, whose symbol $p$ extends holomorphically to a tubular neighborhood of the real phase space and converges sufficiently fast to 1 , so that the determinant is welldefined. We show that the logarithm of the modulus of this determinant is bounded by $(2 \pi h)^{-n}(I(\Lambda, p)+o(1)), h \rightarrow 0$, where $I(\Lambda, p)$ is the integral of $\log |p|$ over $\Lambda$ and $\Lambda$ belongs to a class of deformations of the real phase space on which the restriction of the symplectic form is real and non-degenerate. We show that $I$ is a Lipschitz function of $\Lambda$ and we study its differential and sometimes its Hessian. Under weak additional assumptions, we show that critical "points" $\Lambda$ of the functional $I$ are infinitesimal minima to infinite order.
\end{abstract}

0. Introduction. In the theory of non-selfadjoint operators, determinants play an important role (see for instance [GoKr], [MaMa]) and recent developments in the theory of resonances have brought new interest in operator determinants (see for instance $[\mathrm{Me}],[\mathrm{Zw} 3],[\mathrm{Vo}]$, for the use of determinants in getting upper bounds on the density of resonances, and [GuZw], [Zw1], [Sj4] for the use in getting traceformulae).

There does not seem to be many works devoted to estimates and asymptotics of determinants by direct microlocal methods, and the present work is an attempt in that direction. An interesting feature is that the determinant of an operator is independent of the choice of norm of the Hilbert space where the operator acts, and sometimes to some extent even of the space itself. In the study of resonances the possibility of changing the ambient Hilbert space has played an important role since the beginning of the method of complex scaling and many variants of that method have been used, including phase space versions (see $[\mathrm{AgCo}],[\mathrm{BaCo}],[\mathrm{HeSj}],[\mathrm{SjZw}]$ ). To get optimal estimates, one often has to choose a Hilbert space norm adapted to the problem (see for instance $[\mathrm{HeSj}],[\mathrm{Sj} 5],[\mathrm{Ze}]$ ) and this choice is often the result of phase space analysis.

The work [Da] gives examples of paradoxes encountered when not looking for the optimal Hilbert space. In that work, Davies considers a non-selfadjoint Schrödinger operator on $\mathbf{R}$ with a complex valued potential and shows that one can obtain open sets of "pseudo spectral" values $z \in \mathbf{C}$ for which the norm of the resolvent (when it exists) is exponentially large, while for this class of operators, the true spectrum is discrete and confined to a smaller $h$-independent set. The explanation of this (see also [Zw2]) is quite obvious to specialists in partial differential equations: If we consider more generally an $h$-pseudodifferential operator $p\left(x, h D_{x}\right)$ on $\mathbf{R}^{n}$ with symbol $p(x, \xi)$ in a suitable class, and if $\rho_{0}=\left(x_{0}, \xi_{0}\right) \in \mathbf{R}^{2 n}$ is a point where the Poisson bracket satisfies $\frac{1}{i}\{p, \bar{p}\}\left(\rho_{0}\right)>0$, then if $z_{0}=p\left(\rho_{0}\right)$, we can construct a WKB-solution $u(x ; h)=a(x ; h) e^{i \phi(x) / h}$, with $\operatorname{Im} \phi(x) \geq 0$ with equality precisely at $x_{0}$ and where $a$ is an amplitude, such that $\|u\|_{L^{2}}=1,\left\|\left(P-z_{0}\right) u\right\|_{L^{2}}=\mathcal{O}\left(h^{\infty}\right)$ (i.e. $\mathcal{O}_{N}\left(h^{N}\right)$ for every $N)$. (See [Hö2] for this result in the high frequency setting. The modifications for the proof in the semi-classical framework are obvious. Also, if $p(x, h D)$ is an analytic $h$ -

*Received January 29, 2001; accepted for publication September 19, 2001.

$\dagger$ Dept. of Mathematics, University of Lund, Box 118, S-22100 Lund, Sweden.

‡Centre de Mathématiques, Ecole Polytechnique, F-91128 Palaiseau cedex, France and URM 7640, CNRS. 
pseudodifferential operator in a suitable class, for instance a differential operator with analytic coefficients, then $\mathcal{O}\left(h^{\infty}\right)$ can be sharpened to $\mathcal{O}\left(e^{-1 /(C h)}\right)$ for some $C>0$. See [Sj1] for the additional information needed about the WKB method with analytic symbols.) While the closure of the set $p\left(\mathbf{R}^{2 n}\right)$ will contain all limit points of the spectrum, when $h \rightarrow 0$, there is no reason to expect equality in general. In the case of an analytic $h$-pseudodifferential operator, say when $p$ extends to a bounded holomorphic function in a tubular neighborhood of $\mathbf{R}^{2 n}$, one is tempted (as in the method of complex scaling and its variants) to look for some other Hilbert space norm for which the pseudo-spectrum becomes smaller. There are ways of doing such modifications of the space that are associated to deformations of the real phase space. (See [Sj1], [HeSj] and the works on complex scaling already cited). Instead of $\mathbf{R}^{2 n}$, we are led to consider some other closed IR-manifold $\Lambda \subset \mathbf{C}^{2 n}$, i.e. a manifold $\Lambda$ of real dimension $2 n$ such that the restriction to $\Lambda$ of the complex symplectic form

$$
\sigma=\sum_{1}^{n} d \xi_{j} \wedge d x_{j}, \quad(x, \xi) \in \mathbf{C}^{2 n}=\mathbf{C}_{x}^{n} \times \mathbf{C}_{\xi}^{n}
$$

is real and non-degenerate (so that $\Lambda$ is I-Lagrangian, i.e. Lagrangian for $\operatorname{Im} \sigma$ and Rsymplectic, i.e. symplectic with respect to the restriction of $\operatorname{Re} \sigma$ ). If $\Lambda$ is close to $\mathbf{R}^{2 n}$ or has some suitable transversality property (as in Theorem 3.6 below), we can find a naturally associated Hilbert space, on which $p\left(x, h D_{x}\right)$ acts a bounded operator and it turns out that the principal symbol is now $p_{\left.\right|_{\Lambda}}$ rather than $p_{\left.\right|_{\mathbf{R}^{2 n}}}$. The idea would then be to try to find $\Lambda$ such that $p(\Lambda)$ is as small as possible. The problem of finding such an optimal $\Lambda$ is probably very difficult and very deep, and it is not excluded that one gets some more complicated set than a smooth manifold. Nevertheless, we think that the problem should be attacked even though a complete success may be remote or even out of reach.

Recent uses of Carleman estimates in semi-classical problems by Lebeau-Robbiano [LeRo] and N. Burq [Bu] are somewhat related to the ideas developed here. Carleman estimates are weighted estimates and when proving such estimates, one effectively replaces $p(x, h D)$ by a conjugation $e^{\phi(x) / h} p(x, h D) e^{-\phi(x) / h}$ with symbol $p_{\phi}(x, \xi)=$ $p\left(x, \xi+i \phi^{\prime}(x)\right)$ for some real-valued function $\phi(x)$. Geometrically, this amounts to replacing the real phase space by the IR-manifold $\Lambda_{\phi}=\left\{\left(x, \xi+i \phi^{\prime}(x)\right) ;(x, \xi) \in\right.$ $\left.\mathbf{R}^{2 n}\right\}$. When proving such estimates one exploits the negativity of the Poisson bracket $\frac{1}{i}\left\{p_{\phi}, \overline{p_{\phi}}\right\}$ on the characteristic set $p_{\phi}(x, \xi)=0$ or equivalently on the set $p^{-1}(0) \cap \Lambda_{\phi}$. (This method is originally due to Hörmander in a different context.) This looks first somewhat contradictary with what we shall do in the present paper, namely look for IR-manifolds on which the Poisson bracket vanishes where $p$ does, but if we consider how the Carleman estimates are used for instance in $[\mathrm{Bu}]$, we see that the sharpest results will be obtained in some kind of a limiting case where the bracket above would be close to 0 .

In this paper we do not try to study the spectral problem, but we apply the same philosophy to determinants of $h$-pseudodifferential operators $p(x, h D)$ for which the symbol tends to 1 sufficiently fast when $(x, \xi) \rightarrow \infty$, so that the operator is of the form $I+q(x, h D)$, with $q(x, h D)$ of trace class (and so that $\operatorname{det} p(x, h D)$ is well-defined $([\mathrm{GoKr}]))$. It should be noticed that in practice the condition that $p \rightarrow 1$ suffiently fast at infinity can be replaced by an ellipticity assumption near infinity. Then one chooses some reference operator $\widetilde{p}(x, h D)$ which is elliptic everywhere (and hence invertible for sufficiently small $h$ ) and with the property that $p(x, \xi) / \widetilde{p}(x, \xi) \rightarrow 1$ sufficiently 
fast when $(x, \xi) \rightarrow \infty$, and the discussion will apply to the relative determinant $\operatorname{det}\left(p(x, h D) \widetilde{p}(x, h D)^{-1}\right)$.

In section 1 and 3 we develop the geometrical and analytical framework for our results. We define a class of IR-manifolds contained in a tubular neighborhood of $\mathbf{R}^{2 n}$ (or of some other fixed linear IR-manifold.) If $\Lambda$ is such a manifold, we define a corresponding $h$-dependent Hilbert space $H(\Lambda)$ where for simplicity we have imposed conditions at infinity that imply that the space coincides with $L^{2}\left(\mathbf{R}^{n}\right)$, while the norm will in general, be equivalent to the standard $L^{2}$ norm only up to exponentially large factors. If $p$ is a bounded holomorphic function in a sufficiently large tube, containing $\mathbf{R}^{2 n}$ and $\Lambda$, we show that $p(x, h D)$ (in Weyl quantization) acts as a uniformly bounded operator in $H(\Lambda)$. If $p(x, \xi)-1=\mathcal{O}\left(\langle(x, \xi)\rangle^{m}\right), m<-2 n$, then $p(x, h D)-1$ is of trace class and $\operatorname{det} p(x, h D)$ is well-defined and independent of whether we consider $p(x, h D)$ as an operator in $L^{2}\left(\mathbf{R}^{n}\right)$ or in $H(\Lambda)$. In sections 2,3 we show that

$$
\log |\operatorname{det} p(x, h D)| \leq(2 \pi h)^{-n}(I(\Lambda, p)+o(1)), h \rightarrow 0,
$$

where

$$
I(\Lambda, p):=\frac{1}{2} \int_{\Lambda} \log (p(\rho) \overline{p(\rho)}) \mu(d \rho) .
$$

Here $\mu(d \rho)=\left|\sigma^{n}\right| /(n !)$ is the symplectic volume element on $\Lambda$. When $p$ is elliptic on $\Lambda$ in the sense that $p(\rho) \neq 0, \rho \in \Lambda$, we have equality in (0.2) and in that case it is easy to see directly that $I(\Lambda, p)$ remains unchanged under small deformations of $\Lambda$ (as long as the global ellipticity is conserved). The major problem is then to choose $\Lambda$ so that $I(\Lambda, p)$ becomes as small as possible and this is the subject of the remainder of the paper. As already pointed out we do not solve this major problem but obtain several results of some independent interest. The discussion also applies to the similar frameworks developed in [HeSj] (see [Ze] for corresponding trace class considerations) and $[\mathrm{Sj} 2]$.

As reviewed in section 1, to smooth deformations $I \ni t \mapsto \Lambda_{t}$ of IR-manifolds (where $I$ is some bounded open interval containing 0 ), we have an associated smooth family of functions $I \ni t \mapsto f_{t} \in C^{\infty}\left(\Lambda_{t} ; \mathbf{R}\right)$ well-defined up to a $t$-dependent constant, such that if we let $f_{t}$ also denote an almost holomorphic extension to a neighborhood of $\Lambda_{t}$ and define a flow $\Phi_{t}$, by $\partial_{t} \Phi_{t}(\rho)=\widehat{i H_{f_{t}}}(\rho)$ with $H_{f_{t}}$ denoting the complex Hamilton field, defined with the help of the complex linear part of the differential of $f_{t}$ and the complex symplectic form, and where $\widehat{i H_{f_{t}}}=i H_{f_{t}}+\overline{i H_{f_{t}}}$ denotes the corresponding real vector field, then $\Lambda_{t}=\Phi_{t}\left(\Lambda_{0}\right)$. The flow $\Phi_{t}$ is not a complex canonical transformation, because of lack of holomorphy, but the restriction $\kappa_{t}: \Lambda_{0} \rightarrow \Lambda_{t}$ becomes a real canonical transformation. In the case of deformations of the class of manifolds used in this paper, the corresponding $f_{t}$ belong to the space $C_{b}^{\infty}\left(\Lambda_{t} ; \mathbf{R}\right)$ of smooth functions on $\Lambda_{t}$ which are bounded at infinity together with all their derivatives.

In section 4 , we show that $I(\Lambda, p)$ is a Lipschitz function with respect to such deformations (where the deformation parameter may also be multi-dimensional, $t \in$ neigh $\left(0, \mathbf{R}^{k}\right)$ (=some neighborhood of 0 in $\left.\mathbf{R}^{k}\right)$ ), and that the corresponding derivative (defined for almost all $t$ ) is given by

$$
" \partial_{t} I\left(\Lambda_{t}, p\right) "=-\int_{\Lambda_{t}}\left\langle[d \arg p]_{\Lambda_{t}}, H_{f_{t}}\right\rangle \mu(d \rho)=\int_{\Lambda_{t}}\left\langle\left[H_{\arg p}\right]_{\Lambda_{t}}, d f_{t}\right\rangle \mu(d \rho) .
$$

Here a step in the proof is to see that the differential form $d \arg p_{\Lambda_{t}}$ and the Hamilton field $H_{\arg p_{\Lambda_{t}}}$, where $p_{\Lambda_{t}}=p_{\left.\right|_{\Lambda_{t}}}$, have $L^{1}$ coefficients (in a suitable sense). By $[d \arg p]_{\Lambda_{t}}$ 
and $\left[H_{\arg p}\right]_{\Lambda_{t}}$ we denote the $L^{1}$ extensions from $\Lambda_{t} \backslash p_{\Lambda_{t}}^{-1}(0)$ to all of $\Lambda_{t}$. The last expression in (0.4) can also be written as

$$
-\int_{\Lambda_{t}} \operatorname{div}\left[H_{\arg p}\right]_{\Lambda_{t}}(\rho) f_{t}(\rho) \mu(d \rho),
$$

where

$$
\operatorname{div}\left[H_{\arg p}\right]_{\Lambda_{t}}
$$

is a distribution of order $\leq 1$ with support in $p_{\Lambda_{t}}^{-1}(0)$ and with integral 0 . (The divergence of a Hamilton field is zero.) The problem of minimizing $I(\Lambda, p)$ can be thought of as a variational problem, and we are then interested in critical points, i.e we would like to find an IR-manifold, such that the distribution (0.6) is zero.

We do not solve the variational problem in this paper, but in section 7 , we show under some additional fairly weak assumptions on $\Lambda=\Lambda_{0}$, that if $I \ni t \mapsto \Lambda_{t}$ is a smooth deformation such that $\Lambda_{0}$ is critical (in the sense that (0.6) vanishes), then for every $N \in \mathbf{N}$, there is a constant $C_{N}$ such that

$$
I\left(\Lambda_{t}, p\right) \geq I\left(\Lambda_{0}, p\right)-C_{N}|t|^{N} .
$$

So, the critical points (with some weak additional assumptions) are infinitesimal minima. This result depends on two observations. The first one is that if $f$ is independent of $t$ and extends to a bounded holomorphic function in a tube, then we can define the IR-manifolds $\Lambda_{t}=\exp \left(\widehat{t i H}_{f}\right)(\Lambda)$ for $t \in$ neigh $(0, \mathbf{C})$, and it is quite easy to see that $I\left(\Lambda_{t}, p\right)$ becomes a subharmonic function of $t$. But if $f_{\Lambda}$ is real, then we see that $\Lambda_{t}$ only depends on Ret, so $I\left(\Lambda_{t}, p\right)$ becomes a convex function of $t$. Hence, if $\Lambda_{0}$ is critical, $I\left(\Lambda_{0}, p\right)$ has to be a minimum for the particular family $I\left(\Lambda_{t}, p\right)$. The above observation can be partially extended to the case when $f$ is only $C^{\infty}$ on $\Lambda_{0}$, and we use it in section 5 to compute second derivatives of $I(\Lambda, p)$ with respect to the deformation parameter, for more general deformations. The second observation is that for a general deformation $I \ni t \mapsto \Lambda_{t}$ of IR-manifolds, we can approximate $\Lambda_{t}$ to infinite order when $t \rightarrow 0$ by the result of an autonomous deformation acting on $\Lambda_{0}$. (The deformation will depend on $t$ but will be approximately autonomous.) Another more technical ingredient in the proof is the approximation of $I(\Lambda, p)$ by

$$
I_{\epsilon}(\Lambda, p)=\frac{1}{2} \int \log \frac{p(\rho) \overline{p(\rho)}+\epsilon^{2}}{1+\epsilon^{2}} \mu(d \rho),
$$

when $\epsilon \searrow 0$. This approximation is used at many places, and some more refined considerations are developed in section 6 .

In section 8 , we consider the case when the differentials of the real and imaginary parts of $p_{\Lambda}$ are linearly independent at every point of $p_{\Lambda}^{-1}(0)$. In this case we see that $I(\widetilde{\Lambda}, p)$ becomes a smooth function of $\widetilde{\Lambda}$ in a neighborhood of $\Lambda$ and the differential is now a Radon measure acting on $f_{t}$ :

$$
\partial_{t} I\left(\Lambda_{t}, p\right)=2 \pi \int_{\Lambda_{t} \cap p^{-1}(0)} f_{t} \frac{i}{2}\left\{p_{\Lambda_{t}}, \overline{p_{\Lambda_{t}}}\right\} \lambda_{t}(d \rho)=2 \pi \int_{\Lambda_{t} \cap p^{-1}(0)} f_{t} \frac{1}{(n-1) !} \sigma^{n-1} .
$$

Here $\lambda_{t}(d \rho)$ denotes the Liouville measure on $p_{\Lambda_{t}}^{-1}(0)$. From this result we see that $\Lambda$ is critical iff $\frac{i}{2}\left\{p_{\Lambda}, \overline{p_{\Lambda}}\right\} \equiv 0$ on $p_{\Lambda}^{-1}(0)$. Notice that this property implies that if 
we let $p(x, h D)^{*}$ denote the adjoint with respect to the scalar product of $H(\Lambda)$, then there exist $h$-pseudodifferential operators $A, B, C$ of order 0 in $h$, such that for the commutator of $p$ and $p^{*}$ :

$$
\frac{1}{h}\left[p(x, h D), p(x, h D)^{*}\right]=A p(x, h D)+B p(x, h D)^{*}+h C .
$$

In other words, in order to minimize $I(\Lambda, p)$ (and the corresponding determinant in view of $(0.2)$ ), we should choose $\Lambda$ so that $p(x, h D): H(\Lambda) \rightarrow H(\Lambda)$ tends to be a normal operator.

In section 8 , we also consider the case when $p$ depends holomorphically on a spectral parameter, and investigate the minimization problem on an infinitesimal level. (In a future paper we return to this situation in the case of dimension 2, and get much more complete results, including the determination of all eigenvalues of the operator in some fixed $h$-independent domain in the complex plane.)

In section 9 , we make some further considerations in the case when $p_{\Lambda}$ is of principal type $\left(d p_{\Lambda} \neq 0\right.$ on $\left.p_{\Lambda}^{-1}(0)\right)$. On one hand we see that there are situations when $\partial_{t} I\left(\Lambda_{t}, p\right)$ is not a Radon measure in $f_{t}$, and in the case when $p_{\Lambda_{0}}$ is real, we see that $I\left(\Lambda_{t}, p\right)$ is in general not differentiable at $t=0$, because of a jump in the derivative at that point. We compute the amount of the jump.

In section 10, we consider two examples of bounds for relative determinants with a spectral parameter

$$
\operatorname{det}\left((p(x, h D)-z)(\widetilde{p}(x, h D)-z)^{-1}\right),
$$

i.e. we study $I\left(\Lambda,(p-z)(\widetilde{p}-z)^{-1}\right)$. If $z$ varies in a region where $\widetilde{p}(x, h D)$ has no spectrum then $\partial_{z} \partial_{\bar{z}}$ applied to the determinant (0.11) is a constant times the positive measure obtained by putting a Dirac measure at each eigenvalue of $p(x, h D)$. It is therefore of interest to consider $\partial_{z} \partial_{\bar{z}} I\left(\Lambda,(p-z)(\widetilde{p}-z)^{-1}\right)$ which is easily seen to be $\geq 0$. (In this section we let $\Lambda$ be constant, while at the end of section 7 , we give an argument that indicates that we still have positivity if we let $\Lambda=\Lambda_{z}$ depend on $z$ in such way that $\Lambda_{z}$ becomes a minimizer.) The first example is when $p_{\Lambda}$ is real, we see here that $\partial_{z} \partial_{\bar{z}} I$ behaves in good agreement with what can be obtained for $(0.11)$ from the Weyl asymptotics of the real eigenvalues. The second example concerns the case when $p_{\Lambda}$ vanishes to precisely the second order at a point. This is related to resonances for a semi-classical Schrödinger operator, generated by a non-degenerate critical point of the potential. Here we also get good agreement with the known exactly computable case of harmonic oscillators.

Many estimates in the case when $\Lambda$ is of limited regularity, did not get room in the present paper and we plan to collect these results in a separate work (different from the one mentioned above).

The contents of the paper is:

1. IR manifolds and their deformations.

2. Determinants of $h$-pseudodifferential operators on $\mathbf{R}^{n}$.

3. $h$-pseudodifferential operators and IR deformations of $\mathbf{R}^{2 n}$.

4. Hölder properties of $I(\Lambda, p), I_{\epsilon}(\Lambda, p)$, the differential w.r.t. $\Lambda$.

5 . Second derivative under non-autonomous flows.

6. Continuity and convergence for the differential of $\Lambda \mapsto I(\Lambda, p)$.

7. Minimality to infinite order of critical points.

8. The codimension 2 case. 
9. The case when $p_{\Lambda_{0}}$ is of principal type.

10. Examples.

Acknowledgement: The authors are greatly indepted to N. Lerner for interesting comments.

1. IR manifolds and their deformations. In this section we recall some more or less well-known facts. Consider first locally in $\mathbf{R}^{2 n}$ a smooth family $\Lambda_{t}, t \in I \subset \mathbf{R}$ of smooth Lagrangian manifolds, so that $\Lambda_{t}$ is Lagrangian for every $t$ in the interval $I$ and of the form $\kappa_{t}\left(\mathbf{R}^{n}\right)$, where $\kappa_{t}(y)$ is smooth in $(t, y)$ and has injective $y$-differential. Let $\nu_{t}\left(\kappa_{t}(y)\right)=\partial_{t}\left(\kappa_{t}\right)(y)$ be the corresponding deformation field, first defined as a section of $T\left(\mathbf{R}^{2 n}\right)_{\Lambda_{t}}$. If $\widetilde{\kappa}_{t}$ is a second family with the same properties relative to the family $\Lambda_{t}$ and $\widetilde{\nu}_{t}$ the corresponding vectorfield, then $\nu_{t}-\widetilde{\nu}_{t} \in T\left(\Lambda_{t}\right)$, so we can define invariantly a deformation field $\nu_{t}$ as a section of the normal bundle $N\left(\Lambda_{t}\right)=\left(T\left(\mathbf{R}^{2 n}\right)_{\left.\right|_{\Lambda_{t}}}\right) / T\left(\Lambda_{t}\right)$.

Let $\sigma=\sum d \xi_{j} \wedge d x_{j}$ be the canonical 2 form on $\mathbf{R}^{2 n}$. We add $t$ as a variable and consider

$$
L:=\left\{(t, x, \xi) ;(x, \xi) \in \Lambda_{t}\right\} \subset \mathbf{R} \times \mathbf{R}^{2 n} .
$$

Differential forms on $\mathbf{R}^{2 n}$ will be considered also as differential forms on $\mathbf{R} \times \mathbf{R}^{2 n}$ in the natural way. Let $\omega$ be a smooth one form on $\mathbf{R}^{2 n}$ with $d \omega=\sigma$. (We can for instance take $\omega=\sum \xi_{j} d x_{j}$.) Since $d \omega_{\left.\right|_{\Lambda_{t}}}=0$, we have $\omega=d f_{t}$ locally on $\Lambda_{t}$, where $f_{t}$ is smooth and well defined up to a $t$ dependent constant. Adjusting the constants we may assume that $f(t, x, \xi)=f_{t}(x, \xi)$ is smooth on $L$. On $L$ we see that the restriction of $\omega$ to each submanifold $t=$ const. is equal to the corresponding restriction of $d f$ and hence

$$
\omega_{\left.\right|_{L}}=d f+q_{t}(x, \xi) d t
$$

for some function $q=q_{t}(x, \xi)$ which is smooth on $L$. If we replace $\omega$ by another primitive $\widetilde{\omega}$ of $\sigma$, then $\widetilde{\omega}=\omega+d w$ for some smooth function $w$, and $f$ above is replaced by $\widetilde{f}$ with $\widetilde{f}_{t}=f_{t}+\left(w_{\left.\right|_{\Lambda_{t}}}\right)$. This does not change $q_{t}$, so we get a function $q_{t}(x, \xi)$ on $L$ which is well defined up to some arbitrary smooth function $C(t)$. We fix such a choice of $q_{t}$ and define

$$
\Lambda=\left\{(t, \tau, x, \xi) ;(x, \xi) \in \Lambda_{t}, \tau=-q_{t}(x, \xi)\right\}
$$

Then

$$
(\tau d t+\omega)_{\left.\right|_{\Lambda}} \simeq\left(-q_{t} d t+\omega\right)_{\left.\right|_{L}}=d f
$$

so

$$
(d \tau \wedge d t+\sigma)_{\left.\right|_{\Lambda}}=d(\tau d t+\omega)_{\left.\right|_{\Lambda}}=0 .
$$

Hence $\Lambda$ is Lagrangian. Let $\widetilde{q}(t, x, \xi)$ be an extension of $q_{t}(x, \xi)$ to $\mathbf{R} \times \mathbf{R}^{2 n}$. Then $\tau+\widetilde{q}$ vanishes on $\Lambda$ so its Hamilton field $\frac{\partial}{\partial t}+H_{\widetilde{q}_{t}}$ is tangent to $\Lambda$. This means that $H_{\widetilde{q}_{t}}=\nu_{t}$ is the deformation field defined earlier. Notice that the choice of extension $\widetilde{q}_{t}$ of $q_{t}$ affects the Hamilton field only by a component which is tangent to $\Lambda_{t}$, so with some slight abuse of notation, we can say that

$$
\nu_{t}=H_{q_{t}}
$$

Here it is understood that the RHS is defined only as a section of the normal bundle of $\Lambda_{t}$. We formulate the main result so far as a lemma: 
LEMMA 1.1. Let $\Lambda_{t} \subset \mathbf{R}^{n} \times \mathbf{R}^{n}, t \in$ neigh $(0, \mathbf{R})$, be a smooth family of Lagrangian manifolds, in the sense that $L$ above is a smooth submanifold. Then locally we can find a smooth real-valued function $q_{t}(x, \xi),(x, \xi) \in \Lambda_{t}$ (on $L$ ) such that if $\widetilde{q}_{t}(x, \xi)$ is any smooth real extension of $q_{t}$ to $\mathbf{R} \times \mathbf{R}^{n} \times \mathbf{R}^{n}$, then $\frac{\partial}{\partial t}+H_{\widetilde{q}_{t}}$ is tangent to $\Lambda$ defined earlier. In other words, $H_{\widetilde{q}_{t}}$ is a local representative for the deformation field of the family $\left\{\Lambda_{t}\right\}$. Moreover, $q_{t}$ is uniquely determined on each $\Lambda_{t}$ up to a t-dependent constant.

If $\Lambda_{t}$ are defined globally as closed manifolds of $\mathbf{R}^{n} \times \mathbf{R}^{n}$ and simply connected, then the $q_{t}$ can be defined globally on $\Lambda_{t}$ and are unique up to a $t$-dependent constant.

We shall apply this discussion to $I$-Lagrangian submanifolds of $\mathbf{C}^{2 n}=\mathbf{C}_{x}^{n} \times \mathbf{C}_{\xi}^{n}$ and we first review some differential-geometric and symplectic notions. On this space we have the complex symplectic 2 -form:

$$
\sigma=\sum d \xi_{j} \wedge d x_{j}
$$

which is a non-degenerate closed (2,0)-form. The corresponding real 2 -forms $\operatorname{Re} \sigma=$ $\frac{1}{2}(\sigma+\bar{\sigma}), \operatorname{Im} \sigma=\frac{1}{2 i}(\sigma-\bar{\sigma})$ are closed and non-degenerate and hence give rise to real symplectic structures on $\mathbf{C}^{2 n}$. A general vectorfield of type $(1,0)$ is a vector field of the type

$$
\nu=\sum_{1}^{n}\left(a_{j}(x, \xi) \frac{\partial}{\partial x_{j}}+b_{j}(x, \xi) \frac{\partial}{\partial \xi_{j}}\right),
$$

where $a_{j}$ and $b_{j}$ are complex functions defined on some open subset of $\mathbf{C}^{2 n}$. To $\nu$ we associate the real vectorfield

$$
\widehat{\nu}:=2 \operatorname{Re} \nu=\nu+\bar{\nu}
$$

which is simply the vector $\left(a_{1}, \ldots, a_{n}, b_{1}, \ldots, b_{n}\right)$ when identifying $\mathbf{C}^{n} \times \mathbf{C}^{n}$ with the underlying real manifold $\mathbf{R}^{2 n} \times \mathbf{R}^{2 n}$. Notice that $\widehat{\nu}$ is the unique real vectorfield with the property that $\nu(f)=\widehat{\nu}(f)$ at every point where the function $f$ is differentiable and satisfies $\bar{\partial} f=0$. (Here we use the standard notation: $d f=\partial f+\bar{\partial} f$, where $\partial f$ and $\bar{\partial} f$ denote the complex linear and antilinear parts of the differential.) If $a_{j}, b_{j}$ are sufficiently smooth, then locally and for $t$ small enough, we can define $\Phi_{t}(\rho)=\exp (t \widehat{\nu})(\rho)$ and we notice that the components $\left(\Phi_{t}(\rho)\right)_{x_{j}},\left(\Phi_{t}(\rho)\right)_{\xi_{j}}$ solve the system of ODEs

$$
\frac{d}{d t}\left(\Phi_{t}(\rho)\right)_{x_{j}}=a_{j}\left(\Phi_{t}(\rho)\right), \frac{d}{d t}\left(\Phi_{t}(\rho)\right)_{\xi_{j}}=b_{j}\left(\Phi_{t}(\rho)\right), \Phi_{0}(\rho)=\rho
$$

Let $f$ be a complex-valued $C^{1}$-function on some open subset of $\mathbf{C}^{2 n}$. We define the complex Hamilton field of $f$, to be the unique complex vectorfield of type $(1,0)$ which satisfies the pointwise relation

$$
\left\langle\sigma, H_{f} \wedge t\right\rangle=-\langle\partial f, t\rangle, t \in T\left(\mathbf{C}^{2 n}\right) \otimes \mathbf{C} .
$$

We have the usual formula:

$$
H_{f}=\sum_{1}^{n}\left(\frac{\partial f}{\partial \xi_{j}} \frac{\partial}{\partial x_{j}}-\frac{\partial f}{\partial x_{j}} \frac{\partial}{\partial \xi_{j}}\right) .
$$


If $g$ is a real-valued $C^{1}$ function on an open subset of $\mathbf{C}^{2 n}$, we define the real Hamilton fields $H_{g}^{\alpha}$, for $\alpha=\operatorname{Re} \sigma, \operatorname{Im} \sigma$, by the usual relation:

$$
\left\langle\alpha, H_{g}^{\alpha} \wedge t\right\rangle=-\langle d g, t\rangle, t \in T\left(\mathbf{C}^{2 n}\right) .
$$

There are some useful relations between the three types of Hamilton fields, when we have some additional information about $f$. The most important case is the one when $d f$ is complex linear at some given point $\rho_{0}$ :

$$
\bar{\partial} f\left(\rho_{0}\right)=0
$$

and in the following calculations, we restrict the attention to that point. Since $\sigma$ is of type $(2,0)$ and $\overline{H_{f}}$ is of type $(0,1)$, we have

$$
\left\langle\sigma, \overline{H_{f}} \wedge t\right\rangle=0, t \in T_{\rho_{0}}\left(\mathbf{C}^{2 n}\right) \otimes \mathbf{C}
$$

From (1.7), (1.10), we get

$$
\left\langle\sigma, H_{f} \wedge t\right\rangle=-\langle d f, t\rangle, t \in T_{\rho_{0}}\left(\mathbf{C}^{2 n}\right) \otimes \mathbf{C} .
$$

Restrict the relations to real tangent vectors $\left(t \in T_{\rho_{0}}\left(\mathbf{C}^{2 n}\right)\right)$ and take sums and differences:

$$
\begin{aligned}
\left\langle\sigma, 2 \operatorname{Re} H_{f} \wedge t\right\rangle & =-\langle d f, t\rangle, \\
\left\langle\sigma, 2 \operatorname{Im} H_{f} \wedge t\right\rangle & =-\frac{1}{i}\langle d f, t\rangle .
\end{aligned}
$$

Taking the real and imaginary parts of these relations, we get

$$
\begin{gathered}
\left\langle\operatorname{Re} \sigma, 2 \operatorname{Re}\left(H_{f}\right) \wedge t\right\rangle=-\langle d \operatorname{Re} f, t\rangle, \\
\left\langle\operatorname{Im} \sigma, 2 \operatorname{Re}\left(H_{f}\right) \wedge t\right\rangle=-\langle d \operatorname{Im} f, t\rangle, \\
\left\langle\operatorname{Re} \sigma, 2 \operatorname{Im}\left(H_{f}\right) \wedge t\right\rangle=-\langle d \operatorname{Im} f, t\rangle, \\
\left\langle\operatorname{Im} \sigma, 2 \operatorname{Im}\left(H_{f}\right) \wedge t\right\rangle=\langle d \operatorname{Re} f, t\rangle,
\end{gathered}
$$

so at points where $\bar{\partial} f=0$ :

$$
\begin{aligned}
& \widehat{H_{f}}=2 \operatorname{Re} H_{f}=H_{\operatorname{Re} f}^{\operatorname{Re} \sigma}=H_{\operatorname{Im} f}^{\operatorname{Im} \sigma} \\
& \widehat{H_{i f}}=-2 \operatorname{Im} H_{f}=H_{\operatorname{Re} f}^{\operatorname{Im} \sigma}=-H_{\operatorname{Im} f}^{\operatorname{Re} \sigma} .
\end{aligned}
$$

Later, it will be useful to have a relation also in the case when $d f\left(\rho_{0}\right)$ is real. From (1.7) we get for $t \in T_{\rho_{0}}\left(\mathbf{C}^{2 n}\right)$ :

$$
\begin{gathered}
\left\langle\sigma, i H_{f} \wedge t\right\rangle=-\left\langle i \partial_{f}, t\right\rangle, \\
\left\langle\sigma, \overline{i H_{f}} \wedge t\right\rangle=0, \\
2\left\langle\sigma, \operatorname{Re}\left(i H_{f}\right) \wedge t\right\rangle=-\langle i \partial f, t\rangle, \\
2\left\langle\bar{\sigma}, \operatorname{Re}\left(i H_{f}\right) \wedge t\right\rangle=\langle i \bar{\partial} f, t\rangle, \\
\left\langle\operatorname{Im} \sigma, 4 \operatorname{Re}\left(i H_{f}\right) \wedge t\right\rangle=-\langle d f, t\rangle,
\end{gathered}
$$


SO

$$
H_{f}^{\operatorname{Im} \sigma}=4 \operatorname{Re}\left(i H_{f}\right)=2 \widehat{i H_{f}}
$$

at every point where $d f$ is real.

A smooth submanifold $\Lambda \subset \mathbf{C}^{2 n}$ is called I-Lagrangian if it is Lagrangian (and hence of real dimension $2 n$ ) with respect to $\operatorname{Im} \sigma$. It is called R-symplectic if it is a symplectic manifold with respect to the restriction $\operatorname{Re} \sigma_{\left.\right|_{\Lambda}}$, i.e. if this 2 -form is non-degenerate. We say that $\Lambda$ is an IR-manifold if $\Lambda$ is both I-Lagrangian and Rsymplectic. It is easy to see that an IR-manifold is totally real of maximal dimension (m.t.r from now on, see [HöWe]) and for such a manifold $\Lambda$ we know that every $f \in C^{\infty}(\Lambda)$ has a smooth extension $\widetilde{f}$ to some neighborhood of $\Lambda$ such that $\bar{\partial} \widetilde{f}$ vanishes to infinite order on $\Lambda$. Moreover this extension is unique modulo functions which vanish to infinite order on $\Lambda$. We say that $\widetilde{f}$ is almost holomorphic (a.h.) at $\Lambda$. We recall that the notion of almost holomorphic extensions was introduced by $L$. Hörmander [Hö1].

Let $\Lambda_{t}$ be a smooth family of IR-manifolds. Let $q_{t}$ be the corresponding smooth family as in Lemma 1.1, now with $\operatorname{Im} \sigma$ as the real symplectic form. Let $f_{t}=\widetilde{q}_{t}+i r_{t}$ be an a.h. extension of $q_{t}$, so that $\widetilde{q}_{t}$ is a smooth real extension of $q_{t}$. Then (1.19) applies and we get at the points of $\Lambda_{t}$ a corresponding deformation field,

$$
\nu_{t}=\widehat{i H_{f_{t}}}=H_{\widetilde{q}_{t}}^{\operatorname{Im} \sigma}=-H_{r_{t}}^{\operatorname{Re} \sigma}
$$

Since the differential of $f_{t}$ is uniquely determined by that of $q_{t}$ at every point of $\Lambda_{t}$, we see that (1.21) gives a unique section in $\left.T\left(\mathbf{C}^{2 n}\right)\right|_{\Lambda_{t}}$ and not just a normal vector. Integrating $\nu_{t}$, we get local diffeomorphisms $\kappa_{t, s}: \Lambda_{s} \rightarrow \Lambda_{t}$ and from the last expression in (1.21), it follows that $\kappa_{t, s}^{*}\left(\operatorname{Re} \sigma_{\left.\right|_{\Lambda_{t}}}\right)=\operatorname{Re} \sigma_{\left.\right|_{\Lambda}}$, so $\kappa_{t, s}$ are real canonical transformations, i.e. canonical transformations between the real symplectic manifolds $\Lambda_{s}$ and $\Lambda_{t}$.

Finally we notice that $\nu_{t}$ in (1.21) is the realization of the deformation field as a section in $J T\left(\Lambda_{t}\right)$, where $J$ denotes the mapping of $T \mathbf{C}^{n}$ into itself, induced by multiplication by the imaginary unit $i$. Indeed, $\widehat{i \nu}=J \nu$ for every $(1,0)$-type vectorfield $\nu$ and since $f_{t}$ is real on $\Lambda_{t}$, we see that $\widehat{H_{f_{t}}}$ is the vector field tangent to $\Lambda_{t}$, given by

$$
\widehat{H_{f_{t}}}=H_{f_{t_{\mid} \Lambda_{t}}}^{\sigma_{\Lambda_{t}}}
$$

\section{Determinants of pseudodifferential operators on $\mathbf{R}^{n}$.}

If $m \in C^{\infty}\left(\mathbf{R}^{2 n} ;\right] 0, \infty[)$, we denote by $S(m)$ the space of all $a \in C^{\infty}\left(\mathbf{R}^{2 n}\right)$ such that

$$
\partial_{x}^{\alpha} \partial_{\xi}^{\beta} a(x, \xi)=\mathcal{O}_{\alpha, \beta}(m(x, \xi)),
$$

uniformly on $\mathbf{R}^{2 n}$ for all multiindices $\alpha$ and $\beta$. We will always assume that $m$ is an order function in the sense that $m(x, \xi) \leq C_{0}\langle(x, \xi)-(y, \eta)\rangle^{N_{0}} m(y, \eta)$ for some fixed positive constants $C_{0}, N_{0}$, where we use the notation $\langle(x, \xi)\rangle=\left(1+|(x, \xi)|^{2}\right)^{1 / 2}$. For a symbol $a=a(x, \xi ; h)$ depending on $\left.h \in] 0, h_{0}\right], h_{0}>0$, we say that $a \in S(m)$, if (2.1) holds uniformly w.r.t. $h$. Most of the time such symbols will be of the type $a=a_{0}(x, \xi)+h r(x, \xi ; h)$ with $a_{0}, r \in S(m)$. We then call $a_{0}$ the principal part or the principal symbol of $a$. 
If $a \in S(m)$, we denote by Op (a) the corresponding $h$-Weyl quantization:

$$
\operatorname{Op}(a) u(x)=\frac{1}{(2 \pi h)^{n}} \iint e^{\frac{i}{h}(x-y) \cdot \theta} a\left(\frac{x+y}{2}, \theta ; h\right) u(y) d y d \theta,
$$

and recall (see for instance $[\mathrm{DiSj}]$ ) that $\mathrm{Op}(a)$ is continuous $\mathcal{S}\left(\mathbf{R}^{n}\right) \rightarrow \mathcal{S}\left(\mathbf{R}^{n}\right)$, $\mathcal{S}^{\prime}\left(\mathbf{R}^{n}\right) \rightarrow \mathrm{S}^{\prime}\left(\mathbf{R}^{n}\right)$. If $m=1$, then $\mathrm{Op}(a)$ is $\mathcal{O}(1): L^{2} \rightarrow L^{2}$. If $m$ is integrable, then $\mathrm{Op}(a)$ is of trace class and the corresponding trace class norm satisfies $\|\mathrm{Op}(a)\|_{\operatorname{tr}}=\mathcal{O}(1) h^{-n}$. The trace is given by

$$
\operatorname{tr} \mathrm{Op}(a)=\frac{1}{(2 \pi h)^{n}} \iint a(x, \theta ; h) d x d \theta .
$$

Let $m \in S(m)$ be integrable and let $p \in S(1)$ be of the form

$$
p=1+a, a \in S(m),
$$

with principal symbol $p_{0}=1+a_{0}$, where $a_{0}$ is the principal symbol of $a$. Then $\operatorname{det}(\mathrm{Op}(p))$ is well defined, and if $I \ni t \mapsto a^{t} \in S(m)$ is a mapping of class $C^{1}$ defined on some interval $I$, and we put $p^{t}=1+a^{t}$, then we have

$$
\frac{\partial_{t} \operatorname{det} \mathrm{Op}\left(p^{t}\right)}{\operatorname{det} \mathrm{Op}\left(p^{t}\right)}=\operatorname{tr}\left(\mathrm{Op}\left(p^{t}\right)^{-1} \mathrm{Op}\left(\partial_{t} p^{t}\right)\right)
$$

whenever $\mathrm{Op}\left(p^{t}\right)$ has an $L^{2}$ bounded inverse $\left(\mathrm{Op}\left(p^{t}\right)\right)^{-1}$. See also the remark at the end of this section for some related observations. If we assume that $p$ in (2.4) is elliptic in the sense that $p_{0}(x, \xi) \neq 0$ on $\mathbf{R}^{2 n}$, then we can find a map as above with $I=[0,1]$, such that $p^{t}$ is elliptic for all $t$ and with $p^{0}=1, p^{1}=p$. In fact, we only have to notice that $\log p_{0}$ is globally well defined in $S(m)$, and put $p^{t}=p_{0}^{t}+t\left(p-p_{0}\right), p_{0}^{t}=e^{t \log p_{0}}$. For $h>0$ small enough, we then know that Op $\left(p^{t}\right)$ is invertible with inverse $\mathrm{Op}\left(q^{t}\right)$, where $q^{t}=1+b^{t}, b^{t} \in S(m), q_{0}^{t}=1 / p_{0}^{t}$. Combining this with (2.5), (2.3) and standard $h$-pseudodifferential calculus, we get

$$
\begin{aligned}
\frac{\partial_{t} \operatorname{det} \mathrm{Op}\left(p^{t}\right)}{\operatorname{det} \mathrm{Op}\left(p^{t}\right)} & =\frac{1}{(2 \pi h)^{n}}\left(\iint \frac{\partial_{t} p_{0}^{t}}{p_{0}^{t}} d x d \xi+\mathcal{O}(h)\right) \\
& =\frac{1}{(2 \pi h)^{n}}\left(\iint \partial_{t} \log \left(p_{0}^{t}\right) d x d \xi+\mathcal{O}(h)\right)
\end{aligned}
$$

Integrating, we get

$$
\log \operatorname{det} \mathrm{Op}(p)=\frac{1}{(2 \pi h)^{n}}\left(\iint \log \left(p_{0}\right) d x d \xi+\mathcal{O}(h)\right) .
$$

In this identity, we use the natural branch of the logarithm obtained by continuous deformation with $\log (1)=0$. In the RHS, we have a natural deformation from $(x, \xi)=$ $\infty$, while a priori, we need to use the specific deformation $\mathrm{Op}\left(p^{t}\right)$ in order to define the LHS. It is clear however that these two branches of $\log \left(p_{0}\right)$ coincide.

We now drop the ellipticity assumption, and derive an upper bound on

$$
\log |\operatorname{det} O p(p)|=\frac{1}{2} \log \operatorname{det}\left(\mathrm{Op}(p)^{*} \mathrm{Op}(p)\right),
$$


where $\operatorname{Op}(p)^{*}$ denotes the adjoint operator. If $\epsilon>0$, the preceding discussion applies with $\mathrm{Op}(p)$ replaced by $\mathrm{Op}(p) * \mathrm{Op}(p)+\epsilon^{2} \mathrm{Op}(q)$, if $q \in S(m)$, Op $(q) \geq 0, q_{0}>0$ near $p_{0}^{-1}(0)$. We get for $h>0$ small enough:

$$
\begin{aligned}
\log |\operatorname{det} \mathrm{Op}(p)| \leq & \frac{1}{2} \log \operatorname{det}\left(\mathrm{Op}(p) * \mathrm{Op}(p)+\epsilon^{2} \mathrm{Op}(q)\right) \\
& =\frac{1}{(2 \pi h)^{n}}\left(\iint \frac{1}{2} \log \left(\left|p_{0}\right|^{2}+\epsilon^{2} q_{0}\right) d x d \xi+\mathcal{O}_{\epsilon}(h)\right)
\end{aligned}
$$

For later convenience, we observe that if $q_{0}=1$ near $p_{0}^{-1}(0)$ or if $\log \left|p_{0}\right|$ is integrable, then

$$
\iint \log \left(\left|p_{0}\right|^{2}+\epsilon^{2} q_{0}\right) d x d \xi-\iint \log \left(\frac{\left|p_{0}\right|^{2}+\epsilon^{2}}{1+\epsilon^{2}}\right) d x d \xi \rightarrow 0, \epsilon \rightarrow 0 .
$$

Notice that

$$
\lim _{\epsilon \rightarrow 0} \iint \log \left(\left|p_{0}\right|^{2}+\epsilon^{2} q_{0}\right) d x d \xi=\iint \log \left|p_{0}(x, \xi)\right|^{2} d x d \xi \in[-\infty,+\infty[.
$$

From (2.8), (2.10), we get

$$
\log |\operatorname{det} \operatorname{Op}(p)| \leq \frac{1}{(2 \pi h)^{n}}\left(\iint \log \left|p_{0}(x, \xi)\right| d x d \xi+o(1)\right), h \rightarrow 0,
$$

provided that the integral to the right is $\neq-\infty$. (It is easy to see that so is the case when $p_{0}$ never vanishes to infinite order at any point. See Lemma 4.2 below.) Note that in the elliptic case, we get by taking real parts in (2.7):

$$
\log |\operatorname{det} \mathrm{Op}(p)|=\frac{1}{(2 \pi h)^{n}}\left(\iint \log \left|p_{0}\right| d x d \xi+\mathcal{O}(h)\right) .
$$

Remark. Let $\mathcal{T}_{1}$ denote the space of trace class operators on $\mathcal{H}=L^{2}\left(\mathbf{R}^{n}\right)$. The mapping

$$
A \mapsto \psi(A)=(\operatorname{det}(I+A))(I+A)^{-1}
$$

which is defined near $0 \in \mathcal{T}_{1}$ extends to an analytic mapping

$$
\psi: \mathcal{T}_{1} \rightarrow \mathcal{L}(\mathcal{H})
$$

where the space to the right is the space of continuous linear operators on $\mathcal{H}$. (The analyticity of $\psi$ at $A_{0} \in \mathcal{T}_{1}$ follows easily from the corresponding property in the finite-dimensional situation if one approximates $A_{0}$ in $\mathcal{T}_{1}$ by a finite rank element.) It is natural to consider $\mathcal{L}(\mathcal{H})$ as the dual of $\mathcal{T}_{1}$ obtained via the pairing $\mathcal{T}_{1} \times \mathcal{L}(\mathcal{H}) \ni$ $(A, B) \mapsto \operatorname{tr}(A B)$. Since $\psi(A)(I+A)=(I+A) \psi(A)=\operatorname{det}(I+A) \cdot I$ it follows in particular that $\operatorname{det}(I+A)$ is analytic in $\mathcal{T}_{1}$. Its differential at $A_{0}$ is an element in $\mathcal{L}(\mathcal{H})$, and we claim that it is equal to $\psi\left(A_{0}\right)$ (cf. $(2.5)$ ). For reasons of analyticity in $A_{0}$ it suffices to verify this when $I+A_{0}$ is invertible. Replacing $A$ by $\left(I+A_{0}\right) A$ in our considerations and using the multiplicative property of the determinant we see that our assertion follows from the fact that $\operatorname{tr}(A)$ is the linear part in the Taylor expansion of $\operatorname{det}(I+A)$ at the origin. 
If $\mathcal{A}$ denotes the Banach space of all functions $a(x, \xi)$ in $\mathbf{R}^{2 n}$ such that

$$
\sum_{|\alpha|+|\beta| \leq N} \iint\left|\partial_{x}^{\alpha} \partial_{\xi}^{\beta} a(x, \xi)\right| d x d \xi<\infty,
$$

where $N$ is a sufficiently large positive integer, then the Weyl quantization $a \mapsto \mathrm{Op}(a)$ is a continuous linear mapping from $\mathcal{A}$ to $\mathcal{T}_{1}$. It follows that $\log |\operatorname{det}(\mathrm{Op}(p))|$, where $p=1+a$, is a plurisubharmonic function in $\mathcal{A}$. The inequality $(2.11)$ provides us with a bound from above for that function in terms of $\iint \log |p(x, \xi)| d x d \xi$, which is also a plurisubharmonic function of $a$.

3. $h$-pseudodifferential operators and IR deformations of $\mathbf{R}^{2 n}$. Let $\Phi_{0}(x)$ be a real quadratic form on $\mathbf{C}^{n}$ which is strictly plurisubharmonic (st.pl.s.h.). Let

$$
H_{\Phi_{0}}=\operatorname{Hol}\left(\mathbf{C}^{n}\right) \cap L^{2}\left(\mathbf{C}^{n} ; e^{-2 \Phi_{0} / h} L(d x)\right),
$$

where $L(d x)$ denotes the Lebesgue measure on $\mathbf{C}^{n} \simeq \mathbf{R}^{2 n}$ and $\operatorname{Hol}\left(\mathbf{C}^{n}\right)$ is the space of all holomorphic functions on $\mathbf{C}^{n}$. We recall (see for instance the beginning of [Sj2]) that there exists a unitary operator (a generalized Bargman transform) $W: L^{2}\left(\mathbf{R}^{n}\right) \rightarrow$ $H_{\Phi_{0}}$, given by

$$
W u(x)=C h^{-3 n / 4} \int e^{\frac{i}{h} \phi_{0}(x, y)} u(y) d y
$$

where $\phi_{0}$ is quadratic and holomorphic on $\mathbf{C}_{x}^{n} \times \mathbf{C}_{y}^{n}$ with

$$
\operatorname{det} \partial_{x} \partial_{y} \phi_{0}(x, y) \neq 0, \operatorname{Im} \partial_{y}^{2} \phi_{0}>0
$$

and such that the complex canonical tranformation

$$
\kappa_{W}:\left(y,-\partial_{y} \phi_{0}(x, y)\right) \mapsto\left(x, \partial_{x} \phi_{0}(x, y)\right)
$$

maps $\mathbf{R}^{2 n}$ onto

$$
\Lambda_{\Phi_{0}}:=\left\{\left(x, \frac{2}{i} \frac{\partial \Phi_{0}(x)}{\partial x}\right) ; x \in \mathbf{C}^{n}\right\} .
$$

If we define $\mathbf{R}^{2 n} \ni(y(x), \eta(x))=\kappa_{W}^{-1}\left(x, \frac{2}{i} \partial_{x} \Phi_{0}(x)\right)$, then it follows that

$$
\begin{gathered}
-\left(\partial_{y} \phi_{0}\right)(x, y(x))=\eta(x),\left(\partial_{x} \phi_{0}\right)(x, y(x))=\frac{2}{i} \partial_{x} \Phi_{0}(x), \\
-\operatorname{Im} \phi_{0}(x, y(x))=\Phi_{0}(x), \Phi_{0}(x)+\operatorname{Im} \phi_{0}(x, y) \sim+|y-y(x)|^{2} .
\end{gathered}
$$

We also recall that if $a \in C_{b}^{\infty}\left(\Lambda_{\Phi_{0}}\right)$ (meaning that $a \in C^{\infty}\left(\Lambda_{\Phi_{0}}\right)$ is bounded together with all its derivatives to all orders), then

$$
\mathrm{Op}(a) u(x)=\frac{1}{(2 \pi h)^{n}} \iint e^{\frac{i}{h}(x-y) \cdot \theta} a\left(\frac{x+y}{2}, \theta\right) u(y) d y d \theta
$$

can be defined as a bounded operator $H_{\Phi_{0}}\left(\mathbf{C}^{n}\right) \rightarrow H_{\Phi_{0}}\left(\mathbf{C}^{n}\right)$, by choosing the only possible integration contour: $\theta=\frac{2}{i} \partial_{x} \Phi_{0}\left(\frac{x+y}{2}\right)$. More generally, if $m \in \mathbf{R}$ and $a \in S\left(\langle(x, \xi)\rangle^{m}\right)$ in the sense that $\langle(x, \xi)\rangle^{-m} \nabla^{k} a$ is bounded for every $k \in \mathbf{N}$, then we can still define Op $(a)$ by the oscillatory integral (3.6) to be a bounded operator 
$H_{\Phi_{0}}\left(\langle x\rangle^{k}\right) \rightarrow H_{\Phi_{0}}\left(\langle x\rangle^{k-m}\right)$ where $H_{\Phi_{0}}\left(\langle x\rangle^{k}\right)=\operatorname{Hol}\left(\mathbf{C}^{n}\right) \cap L^{2}\left(\mathbf{C}^{n} ;\langle x\rangle^{2 k} e^{-2 \Phi_{0} / h} L(d x)\right)$ is equipped with the natural norm. (We have bounds on the operator norms which are uniform in $h$. See [Sj2].) The standard metaplectic invariance for the Weyl quantization holds in this setting and we get

$$
W^{-1} \mathrm{Op}(a) W=\mathrm{Op}(b), b=a \circ \kappa_{W} .
$$

We also recall that the orthogonal projection $\Pi_{0}: L_{\Phi_{0}}^{2} \rightarrow H_{\Phi_{0}}$ is given by

$$
\Pi_{0} u(x)=\frac{C}{h^{n}} \int e^{\frac{2}{h} \Psi_{0}(x, y)} u(y) e^{-\frac{2}{h} \Phi_{0}(y)} L(d y),
$$

where $\Psi_{0}(x, y)$ is the unique quadratic form on $\mathbf{C}_{x}^{n} \times \mathbf{C}_{y}^{n}$ which is holomorphic in $x$ and anti-holomorphic in $y$ (from now on hol-a-hol) and satisfies

$$
\Psi_{0}(x, x)=\Phi_{0}(x) .
$$

(We recall from $[\mathrm{Sj} 1,2]$ that this is obtained by writing the identity operator as an $h$-pseudodifferential operator

$$
u \mapsto \frac{\widetilde{C}}{h^{n}} \iint e^{\frac{2}{h}\left(\Psi_{0}(x, \bar{\theta})-\Psi_{0}(y, \bar{\theta})\right)} u(y) d y d \theta,
$$

and choosing the integration contour $\theta=\bar{y}$.)

Let $\widetilde{W} \subset \subset \subset \subset \subset \mathbf{C}^{n}$ be convex open neighborhoods of 0 . Let $\Phi \in C^{\infty}\left(\mathbf{C}^{n} ; \mathbf{R}\right)$ with $\nabla \Phi(x)-\nabla \Phi_{0}(x) \in C_{b}^{\infty}\left(\mathbf{C}^{n}\right):=S\left(\mathbf{C}^{n}, 1\right)$, so that $\nabla \Phi-\nabla \Phi_{0}$ and its derivatives are all bounded. Define

$$
\Lambda_{\Phi}=\left\{\left(x, \frac{2}{i} \frac{\partial \Phi}{\partial x}(x)\right) ; x \in \mathbf{C}^{n}\right\}
$$

and assume that

$$
\frac{2}{i} \frac{\partial \Phi}{\partial x}(x)-\frac{2}{i} \frac{\partial \Phi_{0}}{\partial x}(x) \in \widetilde{W}, x \in \mathbf{C}^{n},
$$

so that $\Lambda_{\Phi} \subset \Lambda_{\Phi_{0}}+\{0\} \times \widetilde{W}$.

Let $p(x, \xi)$ be holomorphic in $\Lambda_{\Phi_{0}}+\{0\} \times W$ with $p(x, \xi)=\mathcal{O}\left(\langle(x, \xi)\rangle^{m}\right)$ for some $m \in \mathbf{R}$. We define $\mathrm{Op}(p)$ as in (3.6) and get a uniformly bounded operator

$$
\mathrm{Op}(p): H_{\Phi_{0}}\left(\langle x\rangle^{k}\right) \rightarrow H_{\Phi_{0}}\left(\langle x\rangle^{k-m}\right),
$$

where we recall that

$$
H_{\Phi_{0}}\left(\langle x\rangle^{k}\right):=\operatorname{Hol}\left(\mathbf{C}^{n}\right) \cap L^{2}\left(\mathbf{C}^{n} ;\langle x\rangle^{2 k} e^{-2 \Phi_{0}(x) / h} L(d x)\right) .
$$

This only requires that $p_{\Lambda_{\Phi_{0}}}:=p_{\left.\right|_{\Lambda_{\Phi_{0}}}} \in S\left(\Lambda_{\Phi_{0}},\left\langle(x, \xi\rangle^{m}\right)\right.$. We now exploit the holomorphy assumtion and make contour deformations. Let

$$
\theta_{\Phi}(x, y)=\int_{0}^{1} \frac{2}{i} \frac{\partial \Phi}{\partial x}((1-t) x+t y) d t
$$


and notice that

$$
\theta_{\Phi_{0}}(x, y)=\frac{2}{i} \frac{\partial \Phi_{0}}{\partial x}\left(\frac{x+y}{2}\right)
$$

so that

$$
\theta_{\Phi}(x, y)-\frac{2}{i} \frac{\partial \Phi_{0}}{\partial x}\left(\frac{x+y}{2}\right) \in \widetilde{W}, x, y \in \mathbf{C}^{n} .
$$

As in $[\mathrm{Sj} 1,2]$, we can replace the contour $\theta=\theta_{\Phi_{0}}(x, y)$ in $(3.6)$ (with $a=p$ ) by

$$
\theta=\theta_{\Phi}(x, y)+\frac{i}{C} \frac{\overline{x-y}}{\langle x-y\rangle},
$$

where $C>0$ is large enough. It follows that

$$
\mathrm{Op}(p)=\mathcal{O}(1): H_{\Phi}\left(\langle x\rangle^{k}\right) \rightarrow H_{\Phi}\left(\langle x\rangle^{k-m}\right),
$$

uniformly in $h$, where $H_{\Phi}\left(\langle x\rangle^{k}\right)$ is defined as in (3.12).

Now add the assumption that

$$
\partial_{\bar{x}} \partial_{x} \Phi \geq \text { Const. }>0
$$

so that $\Lambda_{\Phi}$ is an IR manifold. We shall describe Op $(p)$ as a kind of Toeplitz operator in the spirit of $[\mathrm{BoSj}]$ and start by studying the asymptotics of the orthogonal projection

$$
\Pi_{\Phi}: L^{2}\left(e^{-2 \Phi / h}\right) \rightarrow H_{\Phi}(1) .
$$

Let $\psi(x, y) \in C^{\infty}\left(\mathbf{C}_{x}^{n} \times \mathbf{C}_{y}^{n}\right)$ be almost holomorphic in $x$ and almost anti-holomorphic in $y$ at the diagonal $\operatorname{diag}\left(\mathbf{C}^{n} \times \mathbf{C}^{n}\right)$, such that

$$
\begin{gathered}
\psi(x, x)=\Phi(x), \partial_{\bar{x}} \psi, \partial_{y} \psi=\mathcal{O}_{N}\left(|x-y|^{N}\right), \forall N, \\
\nabla^{2} \psi \in C_{b}^{\infty}, \partial_{\bar{x}} \psi, \partial_{y} \psi \in C_{b}^{\infty} .
\end{gathered}
$$

For the last part of (3.19), we write $\Phi=\Phi_{0}+f$, where $\nabla f \in C_{b}^{\infty}$ and take $\psi=$ $\Psi_{0}+\tilde{f}$, where $\tilde{f}$ is an almost holomorphic-anti-holomorphic extension of $f$ (viewed as a function on $\left.\operatorname{diag}\left(\mathbf{C}^{n} \times \mathbf{C}^{n}\right)\right)$ with $\partial_{\bar{x}} \widetilde{f}, \partial_{y} \tilde{f} \in C_{b}^{\infty}$. It is wellknown and easy to check that

$$
\Phi(x)+\Phi(y)-2 \operatorname{Re} \psi(x, y) \sim|x-y|^{2},
$$

uniformly for $|x-y| \leq 1 / C$, if $C>0$ is large enough.

Put

$$
Z_{j}=h \partial_{\bar{x}_{j}}+\partial_{\bar{x}_{j}} \Phi, Z_{j}^{*}=-h \partial_{x_{j}}+\partial_{x_{j}} \Phi,{ }^{t} Z_{j}=-h \partial_{\bar{x}_{j}}+\partial_{\bar{x}_{j}} \Phi, t Z_{j}^{*}=h \partial_{x_{j}}+\partial_{x_{j}} \Phi .
$$

If $F(x, y)=2 \psi(x, y)-\Phi(x)-\Phi(y)$, we get

$$
\begin{gathered}
Z_{j}\left(x, h D_{x}\right) \circ e^{F(x, y) / h}=e^{F(x, y) / h} \circ\left(h \partial_{\bar{x}_{j}}+2 \partial_{\bar{x}_{j}} \psi(x, y)\right), \\
Z_{j}^{*}\left(x, h D_{x}\right) \circ e^{F / h}=e^{F / h} \circ\left(-h \partial_{x_{j}}+2 \partial_{x_{j}}(\Phi(x)-\psi)\right), \\
{ }^{t} Z_{j}\left(y, h D_{y}\right) \circ e^{F / h}=e^{F / h} \circ\left(-h \partial_{\bar{y}_{j}}+2 \partial_{\bar{y}_{j}}(\Phi(y)-\psi)\right), \\
{ }^{t} Z_{j}^{*}\left(y, h D_{y}\right) \circ e^{F / h}=e^{F / h} \circ\left(h \partial_{y_{j}}+2 \partial_{y_{j}} \psi\right) .
\end{gathered}
$$


We will use these relations only in a region $|x-y| \leq 1 / C$, where we notice that the 0 th order coefficients of the operators in the right most factors, $\left(2 \partial_{\bar{x}_{j}} \psi, 2 \partial_{x_{j}}(\Phi(x)-\psi)\right.$ etc) all belong to $C_{b}^{\infty}$.

Definition. Let $m(x)$ be an order function on $\mathbf{C}^{n}$ (as after (2.1)). An integral operator

$$
K u(x)=\int k(x, y ; h) u(y) d y
$$

will be called $m$-negligible if $k$ is negligible in the sense that

$$
\left|Z_{x}^{\alpha}\left(Z_{x}^{*}\right)^{\beta}\left({ }^{t} Z_{y}\right)^{\gamma}\left({ }^{t} Z_{y}^{*}\right)^{\delta} k(x, y ; h)\right| \leq C_{N, \alpha, \beta, \gamma, \delta} h^{N}\langle x-y\rangle^{-N} m(x), \quad(x, y) \in \mathbf{C}^{2 n}
$$

for all $N \in \mathbf{N}$ and all multiindices $\alpha, \beta, \gamma, \delta \in \mathbf{N}^{n}$. Here we use the subscripts $x, y$ to indicate in which variables the operators $Z, Z^{*}$ etc should act.

Exemple. Let $a(x, y)$ be smooth with support in a sufficiently small neighborhood of $\operatorname{diag}\left(\mathbf{C}^{2 n}\right)$ of the form $|x-y| \leq 1 / \mathcal{O}(1)$ and satisfy $\nabla_{x}^{\alpha} \nabla_{y}^{\beta} a=\mathcal{O}_{N}(1) m(x)\left(|x-y|^{N}+\right.$ $h^{N}$ ) for all $N$ and all multiindices $\alpha, \beta$, where $m$ is an order function. Then

$$
k(x, y ; h)=e^{\frac{1}{h}(2 \psi(x, y)-\Phi(x)-\Phi(y))} a(x, y)
$$

is $m$-negligible. In fact, the preceding computations show that

$$
Z_{x}^{\alpha}\left(Z_{x}^{*}\right)^{\beta}\left({ }^{t} Z_{y}\right)^{\gamma}\left({ }^{t} Z_{y}^{*}\right)^{\delta}(k(x, y ; h))=e^{\frac{1}{h}(2 \psi(x, y)-\Phi(x)-\Phi(y))} a_{\alpha, \beta, \gamma, \delta},
$$

where $a_{\alpha, \beta, \gamma, \delta}$ satisfies the same estimates as $a$ above. It then suffices to use that

$$
\left|e^{\frac{1}{h}(2 \psi(x, y)-\Phi(x)-\Phi(y))}\right| \leq e^{-\frac{1}{C h}|x-y|^{2}} .
$$

Lemma 3.1. Let $K$ be an integral operator as in (3.21). Then $K$ is m-negligible iff

$$
m^{-1} \operatorname{ad}_{x}^{\epsilon} Z^{\alpha}\left(Z^{*}\right)^{\beta} K Z^{\gamma}\left(Z^{*}\right)^{\delta}=\mathcal{O}\left(h^{N}\right): L^{2} \rightarrow L^{2},
$$

for all $\alpha, \beta, \gamma, \delta, \epsilon \in \mathbf{N}^{n}, N \in \mathbf{N}$.

Proof. The kernel of the operator in (3.23) is

$$
m(x)^{-1}(x-y)^{\epsilon} Z_{x}^{\alpha}\left(Z_{x}^{*}\right)^{\beta}\left({ }^{t} Z_{y}\right)^{\gamma}\left({ }^{t} Z_{y}^{*}\right)^{\delta}(k(x, y ; h)),
$$

and if $K$ is $m$-negligible, this kernel is $\mathcal{O}\left(h^{N}\langle x-y\rangle^{-N}\right)$ for all $N \geq 0$, so (3.23) follows.

For the opposite implication, let $x_{0} \in \mathbf{C}^{n}$ and put $\xi_{0}=2 i \partial_{x} \Phi\left(x_{0}\right)$. Then with $f_{j}=\partial_{\bar{x}_{j}} \Phi(x)-\partial_{\bar{x}_{j}} \Phi\left(x_{0}\right)$, we get

$$
\begin{gathered}
Z_{j}=e^{-i \operatorname{Re}\left(\xi_{0} \cdot(\cdot)\right) / h} \circ\left(h \partial_{\bar{x}_{j}}+f_{j}(x)\right) \circ e^{i \operatorname{Re}\left(\xi_{0} \cdot(\cdot)\right) / h}, \\
Z_{j}^{*}=e^{-i \operatorname{Re}\left(\xi_{0} \cdot(\cdot)\right) / h} \circ\left(-h \partial_{x_{j}}+\overline{f_{j}(x)}\right) \circ e^{i \operatorname{Re}\left(\xi_{0} \cdot(\cdot)\right) / h} .
\end{gathered}
$$

Notice that $f_{j}$ and all its derivatives with respect to $x$ are uniformly bounded with respect to $x_{0}$, in a domain $\left|x-x_{0}\right| \leq \mathcal{O}(1)$.

Similarly, put $\eta_{0}=-2 i \partial_{y} \Phi\left(y_{0}\right)$. Then we get

$$
{ }^{t} Z_{j}=e^{-i \operatorname{Re}\left(\eta_{0} \cdot(\cdot)\right) / h} \circ\left(-h \partial_{\bar{y}_{j}}+f_{j}(y)\right) \circ e^{i \operatorname{Re}\left(\eta_{0} \cdot(\cdot)\right) / h},
$$




$$
{ }^{t} Z_{j}^{*}=e^{-i \operatorname{Re}\left(\eta_{0} \cdot(\cdot)\right) / h} \circ\left(h \partial_{y_{j}}+\overline{f_{j}}(y)\right) \circ e^{i \operatorname{Re}\left(\eta_{0} \cdot(\cdot)\right) / h},
$$

Now assume (3.23). Then for $\left(x_{0}, y_{0}\right) \in \mathbf{C}^{n} \times \mathbf{C}^{n},\left|x-x_{0}\right|,\left|y-y_{0}\right| \leq 1$, we reduce $Z, Z^{*}, .$. as above and conclude by letting first $|\alpha|+|\beta|+|\gamma|+|\delta| \leq 4 n+1$, that $m(x)^{-1} e^{i \operatorname{Re}\left(\xi_{0} \cdot x\right) / h} k(x, y ; h) e^{i \operatorname{Re}\left(\eta_{0} \cdot y\right) / h}=\mathcal{O}\left(h^{N}\right)$, i.e. $m(x)^{-1} k(x, y ; h)=\mathcal{O}\left(h^{N}\right)$. In fact, we multiply $k(x, y ; h)$ by $\chi\left(x-x_{0}\right) e^{i \operatorname{Re}\left(\xi_{0} \cdot x\right) / h} \chi\left(y-y_{0}\right) e^{i \operatorname{Re}\left(\eta_{0} \cdot y\right) / h}$, where $\chi \in C_{0}^{\infty}$. Since the $f_{j}$ are uniformly bounded, the corresponding operator will then satisfy (3.23) with the $Z_{j}$ replaced by their leading parts. Repeating this argument with $K$ replaced by $\operatorname{ad}_{x}^{\epsilon} Z^{\alpha}\left(Z^{*}\right)^{\beta} K Z^{\gamma}\left(Z^{*}\right)^{\delta}$, we see that

$$
\frac{1}{m(x)}(x-y)^{\epsilon} Z_{x}^{\alpha}\left(Z_{x}^{*}\right)^{\beta}\left({ }^{t} Z_{y}\right)^{\gamma}\left({ }^{t} Z_{y}^{*}\right)^{\delta} k(x, y)=\mathcal{O}\left(h^{N}\right) .
$$

(We first get this for $\left|x-x_{0}\right|,\left|y-y_{0}\right| \leq 1$, but uniformly with respect to $x_{0}, y_{0}$, which are arbitrary.) (3.22) follows. [

As a second example of negligible operators, we look at the off-diagonal contribution to $\mathrm{Op}(p)$ above, with the contour (3.14). Along this contour we have

$$
d \theta d y= \pm \operatorname{det}\left(\frac{\partial\left(\theta_{\Phi}(x, y)+\frac{1}{C} \frac{\overline{x-y}}{\langle x-y\rangle}\right)}{\partial \bar{y}}\right) d \bar{y} d y=J(x, y) L(d y) .
$$

It is clear that $J \in C_{b}^{\infty}$. The realization of $e^{-\Phi / h} \mathrm{Op}(p) e^{\Phi / h}$ becomes

$$
e^{-\Phi / h} \mathrm{Op}(p) e^{\Phi / h} u(x)=\frac{1}{(2 \pi h)^{n}} \int e^{\frac{1}{h}\left(F(x, y)-\frac{1}{C} \frac{|x-y|^{2}}{\langle x-y\rangle}\right.} q(x, y ; h) u(y) L(d y),
$$

where

$$
\begin{gathered}
F(x, y)=-\Phi(x)+\Phi(y)+2 \int_{0}^{1} \frac{\partial \Phi}{\partial x}((1-t) x+t y) d t \cdot(x-y) \\
q(x, y ; h)=J(x, y) p\left(\frac{x+y}{2}, \theta_{\Phi}(x, y)+\frac{i}{C} \frac{\overline{x-y}}{\langle x-y\rangle}\right)
\end{gathered}
$$

We notice that

$$
\nabla_{x}^{\alpha} \nabla_{y}^{\beta} q(x, y ; h)=\mathcal{O}_{\alpha, \beta}\left(\left\langle\frac{x+y}{2}\right\rangle^{m}\right) .
$$

$F$ is purely imaginary, and if we let $F_{0}$ denote the corresponding function, defined with $\Phi_{0}$ instead of $\Phi$, then $F_{0}$ is quadratic and

$$
\nabla^{k}\left(F-F_{0}\right)=\mathcal{O}(\langle x-y\rangle)
$$

for every $k$. (Recall that $\nabla\left(\Phi-\Phi_{0}\right) \in C_{b}^{\infty}$.) It follows that

$$
\nabla^{k}((\nabla F)(x, y)-(\nabla F)(x, x)), \nabla^{k}(\nabla F(x, y)-\nabla F(y, y))=\mathcal{O}(\langle x-y\rangle) .
$$

We compute for $x=y$ :

$$
\partial_{x} F=\partial_{x} \Phi, \partial_{\bar{x}} F=-\partial_{\bar{x}} \Phi, \partial_{y} F=-\partial_{y} \Phi, \partial_{\bar{y}} F=\partial_{\bar{y}} \Phi,
$$

so if $G(x, y)=F(x, y)-\frac{1}{C} \frac{|x-y|^{2}}{\langle x-y\rangle}$ is the exponent in (3.26), we get

$$
\begin{aligned}
& \partial_{x} G(x, y)=\partial_{x} \Phi(x)+r_{1}(x, y), \partial_{\bar{x}} G(x, y)=-\partial_{\bar{x}} \Phi(x)+r_{2}(x, y), \\
& \partial_{y} G(x, y)=-\partial_{y} \Phi(y)+r_{3}(x, y), \partial_{\bar{y}} G(x, y)=\partial_{\bar{y}} \Phi(y)+r_{4}(x, y),
\end{aligned}
$$


with

$$
\nabla^{k} r_{j}(x, y)=\mathcal{O}(\langle x-y\rangle)
$$

Consequently

$$
\begin{aligned}
& Z_{j, x}\left(e^{G(x, y) / h} q\right)=e^{G(x, y) / h}\left(h \partial_{\bar{x}_{j}}+r_{2, j}(x, y)\right) q, \\
& Z_{j, x}^{*}\left(e^{G(x, y) / h} q\right)=e^{G(x, y) / h}\left(-h \partial_{x_{j}}-r_{1, j}(x, y)\right) q, \\
& { }^{t} Z_{j, y}\left(e^{G(x, y) / h} q\right)=e^{G(x, y) / h}\left(-h \partial_{\bar{y}_{j}}-r_{4, j}(x, y)\right) q, \\
& { }^{t} Z_{j, y}^{*}\left(e^{G(x, y) / h} q\right)=e^{G(x, y) / h}\left(h \partial_{y_{j}}+r_{3, j}(x, y)\right) q .
\end{aligned}
$$

Now (3.29), (3.34) give

$$
Z_{x}^{\alpha}\left(Z_{x}^{*}\right)^{\beta}\left({ }^{t} Z_{y}\right)^{\gamma}\left({ }^{t} Z_{y}^{*}\right)^{\delta}\left(e^{\frac{1}{h} G(x, y)} q\right)=e^{\frac{1}{h} G} \mathcal{O}(1)\langle x-y\rangle^{|\alpha|+|\beta|+|\gamma|+|\delta|}\left\langle\frac{x+y}{2}\right\rangle^{m} .
$$

Using finally that $\left|e^{\frac{1}{h} G(x, y)}\right|=e^{-\frac{1}{C h} \frac{|x-y|^{2}}{\langle x-y\rangle}}$, we conclude that

$$
u \mapsto \frac{1}{(2 \pi h)^{n}} \int e^{\frac{1}{h}\left(F(x, y)-\frac{|x-y|^{2}}{C\langle x-y\rangle}\right)}(1-\chi(x-y)) q(x, y ; h) u(y) L(d y)
$$

is $\langle\cdot\rangle^{m}$-negligible, if $\chi \in C_{0}^{\infty}\left(\mathbf{C}^{n}\right)$ is equal to 1 near 0 .

Formal construction of the orthogonal projection $\Pi_{\Phi}$. Let $\psi$ be as in (3.18-20). Let $a(x, y ; h) \in S\left(\mathbf{C}^{2 n}, 1\right)$ have its support in $|x-y| \leq 1 / C$, with $C$ large enough, and such that

$$
\partial_{\bar{x}} a(x, y ; h), \partial_{y} a(x, y ; h)=\mathcal{O}\left(|x-y|^{\infty}+h^{\infty}\right) .
$$

Consider the operator

$$
A u(x)=(\pi h)^{-n} \int e^{\frac{2}{h} \psi(x, y)} a(x, y ; h) u(y) e^{-2 \Phi(y) / h} L(d y),
$$

which is $\mathcal{O}(1): L^{2}\left(e^{-2 \Phi / h} L(d x)\right) \rightarrow L^{2}\left(e^{-2 \Phi / h} L(d x)\right)$ and satisfies $\bar{\partial} \circ A=\mathcal{O}\left(h^{\infty}\right)$ : $L^{2}\left(e^{-2 \Phi / h}\right) \rightarrow L^{2}\left(e^{-2 \Phi / h}\right)$. Equivalently for the reduced operators, we have

$$
e^{-\Phi / h} A e^{\Phi / h}=\mathcal{O}(1): L^{2} \rightarrow L^{2}, Z_{j} e^{-\Phi / h} A e^{\Phi / h}=\mathcal{O}\left(h^{\infty}\right): L^{2} \rightarrow L^{2} .
$$

The calculations in the first example above show that

$$
Z^{\alpha}\left(Z^{*}\right)^{\beta} e^{-\Phi / h} A e^{\Phi / h} Z^{\gamma}\left(Z^{*}\right)^{\delta}=\mathcal{O}\left(h^{|\alpha|+|\beta|+|\gamma|+|\delta|}\right): L^{2} \rightarrow L^{2},
$$

and applying $\operatorname{ad}_{x}^{\epsilon}$ to this operator, we gain another power $h^{\epsilon / 2}$ in the estimates (3.39). Notice that the composition of $e^{\Phi / h} A e^{\Phi / h}$ with an $m$-negligible operator is $m$-negligible in view of Lemma 3.1.

Formally $A$ is a pseudodifferential operator, for if we put $\widetilde{\psi}(x, y)=\psi(x, \bar{y})$, $\widetilde{a}(x, y)=a(x, \bar{y})$, then $\widetilde{\psi}, \widetilde{a}$ are almost holomorphic on the anti-diagonal $: y=\bar{x}$, and

$$
\begin{aligned}
A u(x) & =\left(\frac{i}{2 \pi h}\right)^{n} \iint e^{\frac{2}{h}(\widetilde{\psi}(x, \bar{y})-\widetilde{\psi}(y, \bar{y}))} \widetilde{a}(x, \bar{y} ; h) u(y) d y d \bar{y} \\
& =\left(\frac{i}{2 \pi h}\right)^{n} \iint_{\theta=\bar{y}} e^{\frac{2}{h}(\widetilde{\psi}(x, \theta)-\widetilde{\psi}(y, \theta))} \widetilde{a}(x, \theta ; h) u(y) d y d \theta .
\end{aligned}
$$


With this in mind, we compute

$$
\begin{gathered}
\left(e^{-\Phi / h} A e^{\Phi / h}\right)^{2} u(x)=(\pi h)^{-n} \int I(x, y ; h) u(y) L(d y), \\
I(x, y ; h)=(\pi h)^{-n} \int e^{(-\Phi(x)+2 \psi(x, z)-2 \Phi(z)+2 \psi(z, y)-\Phi(y)) / h} a(x, z ; h) a(z, y ; h) L(d z) .
\end{gathered}
$$

Because of the support property of $a$, the support of $I$ is contained in a set where $|x-y|$ is small.

We have

$$
\operatorname{Re}(-\Phi(x)+2 \psi(x, z)-2 \Phi(z)+2 \psi(z, y)-\Phi(y)) \sim-\left(|x-z|^{2}+|z-y|^{2}\right) .
$$

Further, when $x=y$, the function

$$
z \mapsto-\Phi(x)+2 \psi(x, z)-2 \Phi(z)+2 \psi(z, y)-\Phi(y)
$$

has the non-degenerate critical point $z=x=y$. We then know from [MeSj] that $I(x, y ; h)$ has an asymptotic expansion, when $h \rightarrow 0$ and to understand the exponent appearing there, we should look for the critical point of the almost holomorphic extension to the complexification of $\mathbf{C}_{z}^{n}$. For that, it is convenient to identify $\mathbf{C}_{z}^{n}$ with the anti-diagonal $\left\{(z, w) \in \mathbf{C}^{2 n} ; w=\bar{z}\right\}$ and write the function (3.43) as

$$
z \mapsto-\Phi(x)+2 \widetilde{\psi}(x, \bar{z})-2 \widetilde{\psi}(z, \bar{z})+2 \widetilde{\psi}(z, \bar{y})-\Phi(y) .
$$

A natural choice of almost holomorphic extension is then

$$
(z, w) \mapsto-\Phi(x)+2 \widetilde{\psi}(x, w)-2 \widetilde{\psi}(z, w)+2 \widetilde{\psi}(z, \bar{y})-\Phi(y) .
$$

We get the critical point $z=x, w=\bar{y}\left(\bmod \mathcal{O}\left(|x-y|^{\infty}\right)\right)$ and the corresponding critical value

$$
-\Phi(x)+2 \widetilde{\psi}(x, \bar{y})-\Phi(y)=-\Phi(x)+2 \psi(x, y)-\Phi(y) .
$$

The stationary phase method gives

$$
I(x, y ; h)=e^{\frac{1}{h}(-\Phi(x)+2 \psi(x, y)-\Phi(y))} b(x, y ; h)+r(x, y ; h),
$$

where $r=\mathcal{O}\left(h^{\infty}\right)$ and $b \sim b_{0}(x, y)+b_{1}(x, y) h+.$. in $S(1)$, and each term in this asymptotic expansion is determined by the behaviour of $(z, w) \mapsto \widetilde{a}(x, w ; h) \widetilde{a}(z, \bar{y} ; h)$ near the critical point $w=\bar{y}, z=x$, so $\partial_{\bar{x}} b, \partial_{y} b=\mathcal{O}\left(|x-y|^{\infty}+h^{\infty}\right)$, and $b_{0}(x, y)=$ $f(x, y) a_{0}(x, y)^{2}$, where

$$
\frac{1}{C} \leq f(x, x) \leq C .
$$

We also notice that if $a \sim a_{0}+h a_{1}+\ldots$, then $b_{j}=2 f a_{0} a_{j}+q_{j}$, where $q_{j}$ depends on $a_{0}, \ldots, a_{j-1}$ only. If we choose $\psi$ such that $\overline{\psi(x, y)}=\psi(y, x)$, as we may, and assume that $\overline{a(x, y ; h)}=a(y, x ; h)$ we achieve that $\overline{I(x, y ; h)}=I(y, x ; h)$.

From (3.39), (3.45), the symbol properties of $b$ and (3.47), we deduce that

$$
Z_{x}^{\alpha}\left(Z_{x}^{*}\right)^{\beta t} Z_{y}^{\gamma}\left({ }^{(t} Z_{y}^{*}\right)^{\delta} r(x, y ; h)=\mathcal{O}\left(h^{N(\alpha, \beta, \gamma, \delta)}\right) .
$$


Combining this with the fact that $r=\mathcal{O}\left(h^{\infty}\right)$, we get

$$
Z_{x}^{\alpha}\left(Z_{x}^{*}\right)^{\beta t} Z_{y}^{\gamma}\left({ }^{t} Z_{y}^{*}\right)^{\delta} r(x, y ; h)=\mathcal{O}\left(h^{\infty}\right)
$$

In fact, using the reduction in the second part of the proof of Lemma 3.1, we see that for $\left|x-x_{0}\right|,\left|y-y_{0}\right| \leq 1, x_{0}=y_{0}$, we have:

$$
\partial_{\bar{x}}^{\alpha} \partial_{x}^{\beta} \partial_{\bar{y}}^{\gamma} \partial_{y}^{\delta}\left(e^{\frac{i}{h}\left(\operatorname{Re}\left(\xi_{0} \cdot x+\eta_{0} \cdot y\right)\right)} r\right)=\mathcal{O}\left(h^{\widetilde{N}(\alpha, \beta, \gamma, \delta)}\right),
$$

while (3.50) is equivalent to

$$
\partial_{\bar{x}}^{\alpha} \partial_{x}^{\beta} \partial_{\bar{y}}^{\gamma} \partial_{y}^{\delta}\left(e^{\frac{i}{h}\left(\operatorname{Re}\left(\xi_{0} \cdot x+\eta_{0} \cdot y\right)\right)} r\right)=\mathcal{O}\left(h^{\infty}\right)
$$

By interpolation inequalities for derivatives, $(\widetilde{3.49})$ and the fact that $r=\mathcal{O}\left(h^{\infty}\right)$, we get $(\widetilde{3.50})$ and hence $(3.50)$ as claimed.

The result (3.50) can be reformulated, as saying that

$$
\begin{aligned}
& \left(e^{-\Phi / h} A e^{\Phi / h}\right)^{2} u(x)= \\
& (\pi h)^{-n} \int e^{\frac{1}{h}(-\Phi(x)+2 \psi(x, y)-\Phi(y))} b(x, y ; h) u(y) L(d y)+e^{-\Phi / h} R e^{\Phi / h} u(x),
\end{aligned}
$$

where $e^{-\Phi / h} R e^{\Phi / h}$ is 1-negligible and

$$
R u(x)=(\pi h)^{-n} \int r(x, y ; h) u(y) L(d y), \operatorname{supp}(r) \subset\left\{(x, y) ;|x-y| \leq \frac{1}{\mathcal{O}(1)}\right\} .
$$

We can now construct $a$ as above with $a(x, x ; h)$ real, $1 / C \leq a_{0}(x, x) \leq C$, such that $b=a+\mathcal{O}\left(|x-y|^{\infty}+h^{\infty}\right)$. Let $\widetilde{\Pi}_{\Phi}$ be the correponding operator as in $(3.38)$. Then

$$
\widetilde{\Pi}_{\Phi}^{2}=\widetilde{\Pi}_{\Phi}+R
$$

for a new operator $R$ with $e^{-\Phi / h} R e^{\Phi / h} 1$-negligible. Further, we may arrange so that $\psi(y, x)=\overline{\psi(x, y)}, a(y, x)=\overline{a(x, y)}$,

$$
\widetilde{\Pi}_{\Phi}^{*}=\widetilde{\Pi}_{\Phi}
$$

where the star indicates that we take the adjoint in $L^{2}\left(e^{-2 \Phi / h} L(d x)\right)$.

We pause in order to recall some general estimates for $\Pi_{\Phi}$ by means of the $\bar{\partial}$ operator, and we will follow the appendix of [Sj2] with some routine elaborations. Put $X_{j}=h^{-1 / 2} Z_{j}=h^{1 / 2} \partial_{\bar{x}_{j}}+h^{-1 / 2} \partial_{\bar{x}_{j}} \Phi, \bar{\partial}_{\Phi}=e^{-\Phi / h} h^{1 / 2} \bar{\partial} e^{\Phi / h}=\sum X_{j} \otimes d_{\bar{x}_{j}}^{\wedge}$, where $\omega^{\wedge}$ denotes the operation of left exterior multiplication by the 1 -form $\omega$. Then $\bar{\partial}_{\Phi}$ is a complex and we put

$$
\Delta_{\Phi}=\bar{\partial}_{\Phi}^{*} \bar{\partial}_{\Phi}+\bar{\partial}_{\Phi} \bar{\partial}_{\Phi}^{*}=\left(\sum X_{j}^{*} X_{j}\right) \otimes 1+\sum_{j, k}\left[X_{j}, X_{k}^{*}\right] d \bar{x}_{j}^{\wedge} d x_{k}^{\rfloor}
$$

where $\left[X_{j}, X_{k}^{*}\right]=2 \partial_{\bar{x}_{j}} \partial_{x_{k}} \Phi \in C_{b}^{\infty}$ and $\left.\omega\right\rfloor$ denotes the transpose of left exterior multiplication by $\omega^{\wedge}$. Here we use the scalar product $\langle x, y\rangle=\frac{1}{2} \operatorname{Re} x \cdot \bar{y}$ on $\mathbf{C}^{n}$ extended to the complexified space, so that $\left\langle d x_{j}, d x_{k}\right\rangle=\left\langle d \bar{x}_{j}, d \bar{x}_{k}\right\rangle=0,\left\langle d x_{j}, d \bar{x}_{k}\right\rangle=\delta_{j, k}$. $\Delta_{\Phi}$ 
conserves the degree of differential forms and if we identify $(0,1)$-forms with functions with values in $\mathbf{C}^{n}$, the restriction to such forms is given by

$$
\Delta_{\Phi}^{(1)}=\left(\sum X_{j}^{*} X_{j}\right) \otimes 1+2 \Phi_{\bar{x} x}^{\prime \prime}
$$

Let $H^{0}=L^{2}$ (here in the vector-valued version $L^{2}\left(\mathbf{C}^{n} ; \mathbf{C}^{n}\right)$ ) and let $H^{1} \subset L^{2}$ be the Hilbert space with norm

$$
\|u\|_{H^{1}}^{2}=\sum\left\|X_{j} u\right\|^{2}+\sum\left\|X_{j}^{*} u\right\|^{2}+\|u\|^{2} .
$$

(More precisely, we define $H^{1}$ as the closure of the Schwartz space $\mathcal{S}$ for the norm above and check that this space coincides with $\left\{u \in L^{2} ; X_{j} u, X_{j}^{*} u \in L^{2}\right\}$.) By construction, the map

$$
H^{1} \ni u \mapsto\left(\left(X_{j} u\right)_{j},\left(X_{j}^{*} u\right)_{j}, u\right) \in\left(L^{2}\right)^{2 n+1}
$$

is an isometry. Let $H^{-1} \subset \mathcal{S}^{\prime}$ be the dual of $H^{1}$. Then the adjoint map

$$
\left(L^{2}\right)^{2 n+1} \ni\left(\left(u_{j}\right)_{j},\left(v_{j}\right)_{j}, u_{0}\right) \mapsto v=u_{0}+\sum X_{j} u_{j}+\sum X_{j}^{*} v_{j} \in H^{-1}
$$

is surjective of norm 1 with a right inverse of norm 1. In particular,

$$
\|v\|_{H^{-1}}^{2}=\inf _{v=u_{0}+\sum X_{j} u_{j}+\sum X_{j}^{*} v_{j}}\left\|u_{0}\right\|^{2}+\sum\left\|u_{j}\right\|^{2}+\sum\left\|v_{j}\right\|^{2} .
$$

We have $H^{1} \subset H^{0} \subset H^{-1}$ with corresponding inequalities for the norms.

An easy calculation shows that

$$
\left[\Delta_{\Phi}^{(1)}, X_{j}\right]=\sum f_{k} X_{k}+g,\left[\Delta_{\Phi}^{(1)}, X_{j}^{*}\right]=\sum \widetilde{f}_{k} X_{k}^{*}+\widetilde{g},
$$

with $f_{k}, \widetilde{f}_{k}, g, \widetilde{g}$ bounded in $C_{b}^{\infty}$ when $h \rightarrow 0$.

In $[\mathrm{Sj} 2]$ we recalled the estimate

$$
\|u\|_{H^{1}} \leq \mathcal{O}(1)\left\|\Delta_{\Phi}^{(1)} u\right\|_{H^{-1}}, u \in \mathcal{S}
$$

implying that $\Delta_{\Phi}^{(1)}: H^{1} \rightarrow H^{-1}$ is bijective with a uniformly bounded inverse for small $h$. Applying this to $X_{j} u$ we get

$$
\left\|X_{j} u\right\|_{H^{1}} \leq \mathcal{O}(1)\left(\left\|X_{j} \Delta_{\Phi}^{(1)} u\right\|_{H^{-1}}+\left\|\left[\Delta_{\Phi}^{(1)}, X_{j}\right] u\right\|_{H^{-1}}\right) .
$$

Using (3.55) and the fact that multiplication by a $C_{b}^{\infty}$ function is bounded $H^{j} \rightarrow H^{j}$, $j=-1,0,1$, we get

$$
\left\|X_{j} u\right\|_{H^{1}} \leq \mathcal{O}(1)\left(\left\|\Delta_{\Phi}^{(1)} u\right\|+\mathcal{O}(1)\|u\|_{H^{1}}\right) \leq \mathcal{O}(1)\left\|\Delta_{\Phi}^{(1)} u\right\| .
$$

Similarly,

$$
\left\|X_{j}^{*} u\right\|_{H^{1}} \leq \mathcal{O}(1)\left\|\Delta_{\Phi}^{(1)} u\right\|
$$

For $s \in \mathbf{N}$, let $H^{s}$ be the Hilbert space of $L^{2}$ functions for which the norm

$$
\|u\|_{H^{s}}^{2}=\sum_{|\alpha|+|\beta| \leq s}\left\|X^{\alpha}\left(X^{*}\right)^{\beta} u\right\|^{2}
$$


is finite. (Again one checks that $\mathcal{S}$ is a dense subspace.) Let $H^{-s} \subset \mathcal{S}^{\prime}$ be the dual space. Then

$$
\text { .. } \subset H^{2} \subset H^{1} \subset H^{0} \subset H^{-1} \subset H^{-2} \subset . .
$$

with corresponding inequalities for the norms. The last estimates show that

$$
\|u\|_{H^{2}} \leq \mathcal{O}(1)\left\|\Delta_{\Phi}^{(1)} u\right\|
$$

Continuing this argument, we get

$$
\|u\|_{H^{s+2}} \leq \mathcal{O}(1)\left\|\Delta_{\Phi}^{(1)} u\right\|_{H^{s}},
$$

for $-1 \leq s \in \mathbf{Z}$. By regularization and bijectivity for $s=1$, we see that $\Delta_{\Phi}^{(1)}: H^{s+2} \rightarrow$ $H^{s}$ is bijective with a uniformly bounded inverse for $s \geq-1$. By duality this extends to all $s \in \mathbf{Z}$. (It helps to use that $\left(\Delta_{\Phi}^{(1)}\right)^{-1}: \mathcal{S} \rightarrow \mathcal{S}$, as we recalled in [Sj2].)

We have $\operatorname{ad}_{x} \Delta_{\Phi}^{(1)}=\mathcal{O}\left(h^{1 / 2}\right): H^{s+1} \rightarrow H^{s}, \operatorname{ad}_{x}^{2} \Delta_{\Phi}^{(1)}: \mathcal{O}(h): H^{s} \rightarrow H^{s}$, $\operatorname{ad}_{x}^{k} \Delta_{\Phi}^{(1)}=0, k \geq 3$. It follows that $\operatorname{ad}_{x}\left(\Delta_{\Phi}^{(1)}\right)^{-1}=-\left(\Delta_{\Phi}^{(1)}\right)^{-1} \operatorname{ad}_{x}\left(\Delta_{\Phi}^{(1)}\right)\left(\Delta_{\Phi}^{(1)}\right)^{-1}=$ $\mathcal{O}\left(h^{1 / 2}\right): H^{s} \rightarrow H^{s+3}$, and more generally,

$$
\operatorname{ad}_{x}^{k}\left(\Delta_{\Phi}^{(1)}\right)^{-1}=\mathcal{O}\left(h^{k / 2}\right): H^{s} \rightarrow H^{s+2+k}
$$

Recall also (see for instance $[\mathrm{Sj} 2]$ ), that

$$
e^{-\Phi / h} \prod_{\Phi} e^{\Phi / h}=1-\bar{\partial}_{\Phi}^{*}\left(\Delta_{\Phi}^{(1)}\right)^{-1} \bar{\partial}_{\Phi}
$$

Since $\bar{\partial}_{\Phi} e^{-\Phi / h} \widetilde{\Pi}_{\Phi} e^{\Phi / h}$ is 1-negligible, we see from (3.59), (3.60) that

$$
e^{-\Phi / h} \Pi_{\Phi} e^{\Phi / h} e^{-\Phi / h} \widetilde{\Pi}_{\Phi} e^{\Phi / h}=e^{-\Phi / h} \widetilde{\Pi}_{\Phi} e^{\Phi / h}+\text { negligible. }
$$

By duality,

$$
e^{-\Phi / h} \widetilde{\Pi}_{\Phi} e^{\Phi / h} e^{-\Phi / h} \Pi_{\Phi} e^{\Phi / h}=e^{-\Phi / h} \widetilde{\Pi}_{\Phi} e^{\Phi / h}+\text { negligible. }
$$

Here "negligible" means some (1-)negligible operator. Put

$$
\widehat{\Pi}_{\Phi}=\Pi_{\Phi} \widetilde{\Pi}_{\Phi} \Pi_{\Phi}
$$

so that

$$
e^{-\Phi / h} \widehat{\Pi}_{\Phi} e^{\Phi / h}=e^{-\Phi / h} \widetilde{\Pi}_{\Phi} e^{\Phi / h}+\text { negligible. }
$$

Then

$$
\begin{aligned}
\widehat{\Pi}_{\Phi} & =\mathcal{O}(1): L^{2}\left(e^{-2 \Phi / h} L(d x)\right) \rightarrow H_{\Phi}, \widehat{\Pi}_{\Phi}^{*}=\widehat{\Pi}_{\Phi}, \\
\widehat{\Pi}_{\Phi}^{2} & =\widehat{\Pi}_{\Phi}+e^{\Phi / h}(\text { negligible }) e^{-\Phi / h} .
\end{aligned}
$$

It follows from (3.63) that the spectrum of $\widehat{\Pi}_{\Phi}$ is concentrated to an $\mathcal{O}\left(h^{\infty}\right)$ neighborhood of $\{0,1\}$. Let $\check{\Pi}_{\Phi}$ be the spectral projection associated to the part of the spectrum close to 1 :

$$
\check{\Pi}_{\Phi}=\frac{1}{2 \pi i} \int_{\gamma}\left(z-\widehat{\Pi}_{\Phi}\right)^{-1} d z,
$$


where $\gamma$ is the positively oriented boundary of the disc $D(1,1 / 2)$.

Proposition 3.2. $\check{\Pi}_{\Phi}=\widehat{\Pi}_{\Phi}+e^{\Phi / h}($ negligible $) e^{-\Phi / h}$.

Proof. As an approximation for $\left(z-\widehat{\Pi}_{\Phi}\right)^{-1}$, we try

$$
R(z)=\frac{1}{z}\left(1-\widehat{\Pi}_{\Phi}\right)+\frac{1}{z-1} \widehat{\Pi}_{\Phi} .
$$

Then, dropping the subscript $\Phi$,

$$
(z-\widehat{\Pi}) R(z)=1-\left(\frac{1}{z}-\frac{1}{z-1}\right) \widehat{\Pi}(1-\widehat{\Pi}) .
$$

Here $\widehat{\Pi}(1-\widehat{\Pi})=e^{\Phi / h}($ negligible $) e^{-\Phi / h}$, and we next verify that if $K$ is of the form $e^{\Phi / h}$ (negligible) $e^{-\Phi / h}$, then $(1-K)^{-1}=1+L$, where $L$ has the same property. Indeed, $L=K+K^{2}+K^{3}+. .=K+K(1-K)^{-1} K$ and we see that $L$ has the required property. It follows that uniformly for $z \in \gamma$ :

$$
(z-\widehat{\Pi})^{-1}=R(z)\left(1-\left(\frac{1}{z}-\frac{1}{z-1}\right) \widehat{\Pi}(1-\widehat{\Pi})\right)^{-1}=R(z)+e^{\Phi / h}(\text { negligible }) e^{-\Phi / h} .
$$

Plugging this into (3.64), we get the proposition.

Notice that we also have

$$
\check{\Pi}_{\Phi}=\widetilde{\Pi}_{\Phi}+e^{\Phi / h}(\text { negl. }) e^{-\Phi / h},
$$

and that by construction, $\check{\Pi}_{\Phi}$ is an orthogonal projection in $L^{2}\left(e^{-2 \Phi / h} L(d x)\right)$ with image contained in $H_{\Phi}$, so that $\check{\Pi}_{\Phi} \prec \Pi_{\Phi}$ in the sense that $\mathcal{R}\left(\check{\Pi}_{\Phi}\right) \subset \mathcal{R}\left(\Pi_{\Phi}\right)$. In particular, $\Pi_{\Phi}-\check{\Pi}_{\Phi}$ is an orthogonal projection.

Proposition 3.3. $\check{\Pi}_{\Phi}=\Pi_{\Phi}$.

Proof. In the case $\Phi=\Phi_{0}$, we have $\check{\Pi}_{\Phi_{0}}=\Pi_{\Phi_{0}}+\mathcal{O}\left(h^{\infty}\right)$, since our construction will reproduce the explicitly known $\Pi_{\Phi_{0}}$. Hence $\check{\Pi}_{\Phi_{0}}=\Pi_{\Phi_{0}}$.

Assume first that $\Phi-\Phi_{0}$ is bounded so that $\Phi-\Phi_{0} \in C_{b}^{\infty}$. Put $\Phi_{t}=t \Phi+$ $(1-t) \Phi_{0}$. Then $L^{2}\left(e^{-2 \Phi_{t} / h}\right), H_{\Phi_{t}}$ are independent of $t$ as spaces and the norms vary continously with $t$. It is therefore clear that $\Pi_{\Phi_{t}}$ is norm-continuous in $t$ in the sense that $\left\|\Pi_{\Phi_{s}}-\Pi_{\Phi_{t}}\right\| \rightarrow 0, s \rightarrow t$. We can make the construction of $\check{\Pi}_{\Phi_{t}}$ so that this operator also becomes norm-continous in $t$. We then have a norm-continous family of orthogonal projections $\Pi_{\Phi_{t}}-\check{\Pi}_{\Phi_{t}}$ which vanishes for $t=0$, and hence for all $t$. Hence we get the proposition when $\Phi-\Phi_{0}$ is bounded.

In the general case, let $R(t)$ be a smooth increasing function: $[0,1[\rightarrow[R(0),+\infty[$ with $R(0) \gg 0, R(t) \rightarrow \infty, t \rightarrow 1$, and put

$$
\Phi^{t}(x)=\Phi_{0}(x)+\left(\Phi(x)-\Phi_{0}(x)\right) \chi\left(\frac{x}{R(t)}\right), 0 \leq t \leq 1,
$$

with the convention that $\Phi^{1}=\Phi$. Here $\chi \in C_{0}^{\infty}(B(0,2) ;[0,1]), \chi(x)=1$ for $x \in$ $B(0,1)$. Then $\nabla^{2} \Phi^{t}$ belongs to a bounded set in $C_{b}^{\infty}, \nabla \Phi^{t}-\nabla \Phi_{0}$ is uniformly bounded. $\Phi^{t}$ is uniformly st.pl.sh. also with respect to $t$. Moreover $\nabla^{k} \Phi_{t} \rightarrow \nabla^{k} \Phi, t \rightarrow 1$, uniformly on every compact, and $\Phi^{t}-\Phi_{0}$ is bounded for every fixed $t<1$. We then know that $\check{\Pi}_{\Phi_{t}}=\Pi_{\Phi^{t}}$ for $t<1$. 
On the other hand, the projections $e^{-\Phi^{t} / h} \check{\Pi}_{\Phi^{t}} e^{\Phi^{t} / h}, e^{-\Phi^{t} / h} \prod_{\Phi^{t}} e^{\Phi^{t} / h}$ are strongly continuous on $[0,1]$, in the first case by examining the construction and restricting to $\mathcal{S}$, in the second case by looking at the representation (3.60), that we also restrict to $\mathcal{S}$. It follows that the two operators coincide also for $t=1$, so we get the proposition in the general case.

The proposition and (3.66) imply that

$$
\Pi_{\Phi}=\widetilde{\Pi}_{\Phi}+e^{\Phi / h}(\text { negligible }) e^{-\Phi / h},
$$

which completes the promised asymptotic study of $\Pi_{\Phi}$.

We next prepare for trace computations. Write (3.38) for $\widetilde{\Pi}_{\Phi}$ :

$$
\widetilde{\Pi}_{\Phi} u(x)=\frac{1}{(\pi h)^{n}} \int e^{\frac{2}{h}(\psi(x, y)-\Phi(y))} f(x, y ; h) u(y) L(d y),
$$

where $f \sim \sum_{0}^{\infty} f_{j}(x, y) h^{j}$ in $S(1), \operatorname{supp} f \subset\{(x, y) ;|x-y| \leq 1 / C\}, \partial_{(\bar{x}, y)} f=$ $\mathcal{O}\left(|x-y|^{\infty}\right)$, and put $\widetilde{\psi}(x, y)=\psi(x, \bar{y}), \widetilde{f}(x, y ; h)=f(x, \bar{y} ; h)$. Recall that $\widetilde{\Pi}_{\Phi}$ is formally the identity operator viewed as a pseudodifferential operator, and that $L(d y)=(i / 2)^{n} d y d \bar{y}:$

$$
1 u(x)=\left(\frac{i}{2 \pi h}\right)^{n} \iint e^{\frac{2}{h}(\widetilde{\psi}(x, \theta)-\widetilde{\psi}(y, \theta))} \widetilde{f}(x, \theta ; h) u(y) d y d \theta .
$$

Use the Kuranishi trick:

$$
2(\widetilde{\psi}(x, \theta)-\widetilde{\psi}(y, \theta))=i(x-y) \cdot \widetilde{\theta}(x, y, \theta), \widetilde{\theta}(x, x, \theta)=\frac{2}{i} \frac{\partial \widetilde{\psi}}{\partial x}(x, \theta),
$$

and

$$
1 u(x)=\frac{1}{(2 \pi h)^{n}} \iint e^{\frac{i}{h}(x-y) \cdot \widetilde{\theta}} u(y) d y d \widetilde{\theta},
$$

to see that

$$
i^{n} \widetilde{f}_{0}(x, \theta) d x d \theta=d x d \widetilde{\theta}(x, x, \theta) .
$$

Put $\theta=\bar{x}$ :

$$
i^{n} \widetilde{f}_{0}(x, \bar{x}) d x d \bar{x}=d x d \xi_{\Lambda_{\Phi}},
$$

since $\widetilde{\theta}(x, x, \bar{x})=\frac{2}{i} \frac{\partial \Phi}{\partial x}(x)$. This can also be written

$$
\frac{1}{\pi^{n}} f_{0}(x, x) L(d x)=\frac{1}{(2 \pi h)^{n}} d x d \xi_{\Lambda_{\Phi}},
$$

where we identify $\mathbf{C}_{x}^{n}$ with $\Lambda_{\Phi}$ by means of the natural projections and $d x d \xi_{\left.\right|_{\Phi}}=$ $\mu(d(x, \xi))$ is the symplectic volume form on $\Lambda_{\Phi}$.

Consider the operator $O p(p)$ in $(3.11),(3.15)$. Noticing that $\Pi_{\Phi}, \widetilde{\Pi}_{\Phi}$ act as bounded operators on $H_{\Phi}\left(\langle x\rangle^{k}\right)$, we write $\mathrm{Op}(p)=\mathrm{Op}(p) \Pi_{\Phi}$, recall that $\Pi_{\Phi}=\widetilde{\Pi}_{\Phi}+$ $e^{\Phi / h}$ (negl.) $e^{-\Phi / h}$, and develop Op $(p) \widetilde{\Pi}_{\Phi}$ by means of stationary phase (after pealing off negligible off-diagonal parts), to see that

$$
\operatorname{Op}(p)=\operatorname{top}(q)+e^{\Phi / h}\left(\langle\cdot\rangle^{m} \text {-negl. }\right) e^{-\Phi / h},
$$




$$
\operatorname{top}(q) u(x)=\frac{1}{(\pi h)^{n}} \int e^{\frac{2}{h}(\psi(x, y)-\Phi(y))} f(x, y ; h) q(x, y ; h) u(y) L(d y) .
$$

Here $q(x, y ; h) \in S\left(\langle\cdot\rangle^{m}\right)$ has its support in $|x-y| \leq 1 / C, q \sim \sum_{0}^{\infty} q_{j}(x, y) h^{j}$ in $S\left(\langle\cdot\rangle^{m}\right), \partial_{\bar{x}, y)} q_{j}=\mathcal{O}\left(|x-y|^{\infty}\langle x\rangle^{m}\right)$, and $q_{0}(x, x)=p\left(x, \frac{2}{i} \frac{\partial \Phi}{\partial x}(x)\right)$.

Let $\widetilde{S}\left(\langle\cdot\rangle^{m}\right)$ be the space of $q(x, y ; h) \in S\left(\langle\cdot\rangle^{m}\right)$ with support in $|x-y| \leq 1 / C$, such that $\partial_{\bar{x}, y} q=\mathcal{O}\left(\left(|x-y|^{\infty}+h^{\infty}\right)\langle x\rangle^{m}\right)$. Let $\widetilde{S}_{\mathrm{cl}}\left(\langle\cdot\rangle^{m}\right)$ be the subspace of $q$ with $q \sim \sum_{0}^{\infty} q_{j}(x, y) h^{j}$ in $S\left(\langle\cdot\rangle^{m}\right)$, where $\partial_{\bar{x}, y} q_{j}=\mathcal{O}\left(|x-y|^{\infty}\langle x\rangle^{m}\right)$.

If $q(x, y) \in C^{\infty}\left(\mathbf{C}^{2 n}\right)$, we define $q^{*}(x, y)=\overline{q(y, x)}$. The self-adjointness of $\widetilde{\Pi}_{\Phi}$ is equivalent to $f^{*}=f$ (assuming that we have chosen $\psi$ with $\psi^{*}=\psi$ ). We have

$$
\operatorname{top}(q)^{*}=\operatorname{top}\left(q^{*}\right)
$$

Moreover, by stationary phase,

$$
\operatorname{top}(q) * \operatorname{top}(q)=\operatorname{top}(r)+e^{\Phi / h}\left(\langle\cdot\rangle^{2 m}-\operatorname{negl} .\right) e^{-\Phi / h},
$$

where $r \in S\left(\langle\cdot\rangle^{2 m}\right), r^{*}=r, r \sim \sum_{0}^{\infty} r_{j} h^{j}, \partial_{(\bar{x}, y)} r_{j}=\mathcal{O}\left(|x-y|^{\infty}\langle x\rangle^{2 m}\right), r_{0}=q_{0}^{*} q_{0}$. (3.74) is a special case of a more general result for the composition of two Toeplitz operators top $(a) \circ$ top $(b)$ that can be formulated in the obvious way.

Recall that if $k \in C_{0}^{\infty}\left(\mathbf{C}^{2 n}\right)$, diamsupp $(k) \leq C_{0}$ and $K u(x)=$ $\int k(x, y) u(y) L(d y)$, then the trace class norm of $K$ is $\leq C_{1}\left(C_{0}, n\right)\|\widehat{k}\|_{L^{1}}$, where $\widehat{k}$ is the Fourier transform of $k$. Moreover the trace of $K$ is given by $\operatorname{tr}(K)=\int k(x, x) L(d x)$.

LEMmA 3.4. Let $m<-2 n$. If $R$ is $\langle\cdot\rangle^{m}$-negligible, then $R$ is of trace class with trace class norm $\leq \mathcal{O}\left(h^{\infty}\right)$.

Proof. Let $r(x, y ; h)$ be the kernel of $R$. For $\left(x_{0}, y_{0}\right) \in \mathbf{C}^{2 n}$, we define $\xi_{0}=$ $2 i \partial_{x} \Phi\left(x_{0}\right), \eta_{0}=-2 i \partial_{y} \Phi\left(y_{0}\right)$ as in the proof of Lemma 3.1. Then for $\left|x-x_{0}\right|,\left|y-y_{0}\right| \leq$ 1 , we have

$$
\nabla_{x}^{k} \nabla_{y}^{\ell}\left(e^{i \operatorname{Re} \xi_{0} \cdot x / h} r(x, y ; h) e^{i \operatorname{Re} \eta_{0} \cdot y / h}\right)=\mathcal{O}(1) h^{N}\left\langle x_{0}-y_{0}\right\rangle^{-N}\left\langle x_{0}\right\rangle^{m},
$$

for every $N \subset \mathbf{N}$ and all multiindices $k, \ell$. (The estimates are uniform in $\left(x_{0}, y_{0}\right)$.) If $\chi \in C_{0}^{\infty}(B(0,1))$, it follows that $\chi\left(\cdot-x_{0}\right) R \chi\left(\cdot-y_{0}\right)$ is of trace class norm $\leq$ $\mathcal{O}(1) h^{N}\left\langle x_{0}-y_{0}\right\rangle^{-N}\left\langle x_{0}\right\rangle^{m}$. Using partitions of unity separately in the $x$ and $y$ variables, we get the lemma.

LEMMA 3.5. Let $m<-2 n$, and let $q \in S\left(\langle\cdot\rangle^{m}\right)$, with support in $|x-y| \leq 1 / C$. Then top $(q)$ is of trace class as an operator on $L^{2}\left(e^{-2 \Phi / h} L(d x)\right)$ and the corresponding trace class norm is $\mathcal{O}\left(h^{-\rho}\right)$, for every $\rho>5 n / 2$. Further,

$$
\operatorname{tr} \operatorname{top}(q)=\frac{1}{(2 \pi h)^{n}}\left(\int_{\Lambda_{\Phi}} q(x, \xi) \mu(d(x, \xi))+\mathcal{O}(h) \int_{\Lambda_{\Phi}}|q(x, \xi)| \mu(d(x, \xi))\right) .
$$

Proof. Let $\chi$ be a cut-off function with support in the unit ball and let $b(x, y)$ be the integral kernel of the operator $B=\chi\left(\cdot-x_{0}\right) e^{-\Phi / h} \operatorname{top}(q) e^{\Phi / h} \chi\left(\cdot-y_{0}\right)$. This has the same trace class norm $\|B\|_{\text {tr }}$ as the operator $B_{1}$ with integral kernel $b_{1}(x, \xi)=$ $e^{i \operatorname{Re}\left(\xi_{0} \cdot x\right) / h} b(x, y) e^{i \operatorname{Re}\left(\eta_{0} \cdot y\right) / h}$. If $2 n<\tau<2 n+1$, then

$$
\left\|B_{1}\right\|_{\operatorname{tr}} \leq C\left\|\widehat{b_{1}}\right\|_{L^{1}} \leq C_{\tau}\left\|\langle D\rangle^{\tau} b_{1}\right\|_{L^{2}} \leq C_{\tau}\left(\left\|\langle D\rangle^{2 n} b_{1}\right\|_{L^{2}}\right)^{2 n+1-\tau}\left(\left\|\langle D\rangle^{2 n+1} b_{1}\right\|_{L^{2}}\right)^{\tau-2 n} .
$$


The estimates above together with (3.20) show that

$$
\left\|\langle D\rangle^{j} b_{1}\right\|_{L^{2}}=\mathcal{O}(1) h^{-n-j}\left\langle x_{0}\right\rangle^{m}\left(\iint \chi\left(x-x_{0}\right) \chi\left(y-y_{0}\right) e^{-c|x-y|^{2} / h} L(d x) L(d y)\right)^{1 / 2}
$$

where $j=2 n, 2 n+1$ and $c$ is a positive constant. The right hand-side may be estimated from above by a constant times $h^{-n-j+n / 2}\left\langle x_{0}\right\rangle^{m} s\left(x_{0}-y_{0}\right)$ where $s$ is a rapidly decreasing function. A combination of these estimates with $j=2 n$ and $j=$ $2 n+1$ gives

$$
\|B\|_{\text {tr }}=\mathcal{O}(1) h^{-n / 2-\tau}\left\langle x_{0}\right\rangle^{m} s\left(x_{0}-y_{0}\right) .
$$

The assertion about the trace class norm of top $(q)$ follows then by a partition of unity if one chooses $\tau=\rho-n / 2$ (assuming that $\rho<5 n / 2+1$ ).

The second statement follows from (3.70).

With $q \in \widetilde{S}\left(\langle\cdot\rangle^{m}\right)$, we put

$$
\operatorname{Top}(q)=\Pi_{\Phi} \operatorname{top}(q) \Pi_{\Phi} .
$$

Then

$$
\left.\operatorname{Top}(q)=\operatorname{top}(q)+e^{\Phi / h}(\langle\cdot\rangle\rangle^{m} \text { negl. }\right) e^{-\Phi / h} .
$$

Notice that if $m<-2 n$, then the trace of $\operatorname{Top}(q)$ is independent of whether we view our operator as acting in $H_{\Phi}$ or in $L^{2}\left(e^{-2 \Phi / h} L(d x)\right)$.

If we choose $q$ as in (3.71), then

$$
\operatorname{Op}(p)=\operatorname{Top}(q)+e^{\Phi / h}\left(\langle\cdot\rangle^{m}-\text { negl. }\right) e^{-\Phi / h} .
$$

We can now adapt the discussion in section 2. Assume that $p(x, \xi)=1+a(x, \xi)$ with $a=\mathcal{O}\left(\langle(x, \xi)\rangle^{m}\right)$ in $\Lambda_{\Phi_{0}}+\{0\} \times W$ for, $m<-2 n$. If we first assume that $p \neq 0$ on $\Lambda_{\Phi}$, we see that $\mathrm{Op}(p): H_{\Phi} \rightarrow H_{\Phi}$ has a bounded inverse

$$
\operatorname{Op}(p)^{-1}=\operatorname{Top}(r)+e^{\Phi / h}(\text { negl. }) e^{-\Phi / h},
$$

where $r \in \widetilde{S}_{\mathrm{cl}}(1), r_{0}(x, x)=1 / p\left(x, \frac{2}{i} \partial_{x} \Phi(x)\right)$. The same holds for Op $\left(p^{t}\right)$, if we define the deformation $p^{t}$ from 1 to $p$ as in section 2 . Using the calculus above, we get the analogue of (2.7):

$$
\log \operatorname{det} \mathrm{Op}(p)=\frac{1}{(2 \pi h)^{n}}\left(\int_{\Lambda_{\Phi}} \log p(\rho) \mu(d \rho)+\mathcal{O}(h)\right),
$$

where $\mu$ is the symplectic volume density on $\Lambda_{\Phi}$. The subsequent argument of section 2 can also be carried over, so if we now allow $p$ to vanish on $\Lambda_{\Phi}$, we get

$$
\log |\operatorname{det} \mathrm{Op}(p)| \leq \frac{1}{(2 \pi h)^{n}}\left(\int_{\Lambda_{\Phi}} \log |p(\rho)| \mu(d \rho)+o(1)\right), h \rightarrow 0 .
$$

We can now state the main result of this section.

THEOREM 3.6. Let $p(x, \xi)$ be holomorphic in an open tubular neighborhood of $\mathbf{R}^{2 n}$ of the form $\mathbf{R}^{2 n}+i W, 0 \in W \subset \subset \mathbf{R}^{2 n}$ and satisfying $p(x, \xi)-1=\mathcal{O}\left(\langle(x, \xi)\rangle^{m}\right)$ there for some $m<-2 n$. Let $F \subset \mathbf{C}^{2 n}$ be a complex Lagrangian space (i.e. a complex subspace of dimension $n$ on which $\sigma$ vanishes) which is strictly negative in the sense 
that $\frac{1}{2 i} \sigma(t \wedge \bar{t})<0, \forall t \in F \backslash\{0\}$. Let $\widetilde{W} \subset \subset W$ be an open convex neighborhood of $0 \in \mathbf{R}^{2 n}$ and let $\Lambda \subset \mathbf{R}^{2 n}+i \widetilde{W}$ be an IR-manifold of the form $\left\{\rho+\ell(\rho) ; \rho \in \mathbf{R}^{2 n}\right\}$, where $\ell \in C_{b}^{\infty}\left(\mathbf{R}^{2 n} ; F\right)$. Then $\sigma_{\left.\right|_{t}}$ is real, where $\Lambda_{t}=\left\{\rho+t \ell(\rho) ; \rho \in \mathbf{R}^{2 n}\right\}, 0 \leq t \leq 1$, and we assume this form to be non-degenerate, uniformly on each $\Lambda_{t}$, uniformly for $t \in[0,1]$. Then

$$
\log \left|\operatorname{det} p^{w}\left(x, h D_{x}\right)\right| \leq \frac{1}{(2 \pi h)^{n}}\left(o(1)+\int_{\Lambda} \log |p| \mu(d \rho)\right), h \rightarrow 0
$$

Proof. Let $\kappa: \mathbf{C}^{2 n} \rightarrow \mathbf{C}^{2 n}$ be a complex linear canoncial transformation with $\kappa(F)=\left\{(x, \xi) \in \mathbf{C}^{2 n} ; x=0\right\}$. The negativity of $F$ implies that this space is of the form

$$
\xi=\frac{\partial f}{\partial x}, x \in \mathbf{C}^{n}
$$

where $f$ is a holomorphic quadratic form with $\operatorname{Im} f^{\prime \prime}<0$. Then it is clear that $\kappa$ is of the form (3.3) where $\phi_{0}$ satisfies (3.2) (noticing that $\phi_{0}(0, y)=-f(y)$ ), and hence that $\kappa=\kappa_{W}$ with $W$ as in (3.1). Then $\kappa\left(\mathbf{R}^{2 n}\right)=\Lambda_{\Phi_{0}}$, where $\Phi_{0}$ is a st.pl.s.h. quadratic form. The assumption about the form of $\Lambda$ implies that $\kappa(\Lambda)=\Lambda_{\Phi}$ with $\nabla \Phi-\nabla \Phi_{0} \in C_{b}^{\infty}$, and more generally, $\kappa\left(\Lambda_{t}\right)=\Lambda_{\Phi_{t}}, \Phi_{t}=t \Phi+(1-t) \Phi_{0}$. Since $\sigma_{\left.\right|_{\Lambda_{t}}}$ is uniformly non-degenerate, we see that $\frac{\partial^{2} \Phi_{t}}{\partial \bar{x} \partial x}$ is uniformly non-degenerate and hence strictly positive, since so is the case for $t=0$.

We recall the metaplectic invariance (3.7), so that $W^{-1} p^{w}(x, h D) W=q^{w}(x, h D)$, where $q=p \circ \kappa^{-1}$. Clearly $\operatorname{det} p^{w}(x, h D)=\operatorname{det} q^{w}(x, h D)$, where we first consider $q^{w}(x, h D)$ as a bounded operator $H_{\Phi_{0}} \rightarrow H_{\Phi_{0}}$. If we assume that $\Phi-\Phi_{0}$ is bounded, then (3.81) follows from (3.80) (with $p$ replaced by $q$ ), since $H_{\Phi}$ is then the same space as $H_{\Phi_{0}}$. In the general case, we can approach $\Phi$ by a sequence of $\widetilde{\Phi}$ such that $\widetilde{\Phi}-\Phi_{0}$ is bounded, $\nabla \widetilde{\Phi} \rightarrow \nabla \Phi$ in $C_{b}^{\infty}, \widetilde{\Phi}$ is uniformly st.pl.s.h. and $\Lambda_{\widetilde{\Phi}}$ stays inside the convex tube $\kappa\left(\mathbf{R}^{2 n}+i \widetilde{W}\right)$. We then have (3.80) uniformly (with $p, \Lambda_{\Phi}$ replaced by $q, \Lambda_{\widetilde{\Phi}}$ ) and passing to the limit we get it also for $\Phi$.

In [Sj2] function spaces are defined associated to IR-manifolds which are obtained by certain global holomorphic deformations and it is showed there how $h$ pseudodifferential operator s act on these spaces and that this action can be pulled back to an action on $H_{\Phi_{0}}$. Under essentially the same assumptions on $P$, we still have the conclusion (3.81).

4. Hölder properties of $I(\Lambda, p), I_{\epsilon}(\Lambda, p)$; the differential w.r.t. $\Lambda$. Let $\Phi_{0}$ and $\Lambda_{\Phi_{0}}$ be as in section 3 . Let $\widetilde{W} \subset \subset W$ be open bounded neighborhoods of 0 in $\Lambda_{\Phi_{0}}$. Let $p$ be holomorphic in $\Lambda_{\Phi_{0}}+i W$ and satisfy

$$
p(x, \xi)-1=\mathcal{O}\left(\langle(x, \xi)\rangle^{m}\right), \text { for some } m<-2 n \text {. }
$$

The IR manifolds $\Lambda$ under consideration in the remainder of this paper will satisfy:

$$
\Lambda \subset \Lambda_{\Phi_{0}}+i \widetilde{W} \text { is closed }
$$

$\Lambda$ is diffeomorphic to $\mathbf{R}^{2 n}$. 
Outside a bounded set, we have $\Lambda=\Lambda_{\Psi}$, with $\Psi-\Phi_{0} \in C_{b}^{\infty},\left(\partial_{\bar{x}} \partial_{x} \Psi\right)^{-1}=\mathcal{O}(1)$.

(From the discussion in section 3 and in this section, it will be clear that the assumption on $\Psi-\Phi_{0}$ can be weakend to: $\Psi^{\prime}-\Phi_{0}^{\prime} \in C_{b}^{\infty}$.)

The last assumption allows us to identify $\Lambda$ near infinity with a neighborhood of infinity of $\mathbf{C}^{n}$ by means of $\mathbf{C}^{n} \ni x \simeq\left(x, \frac{2}{i} \frac{\partial \Psi}{\partial x}(x)\right) \in \Lambda$, and we can therefore define the space $C_{b}^{\infty}(\Lambda)$.

Sometimes we let $\Lambda=\Lambda_{t}$ depend smoothly on $t \in \operatorname{neigh}\left(0 ; \mathbf{R}^{k}\right)$. We then require $\Lambda_{t}$ to fulfill (4.2)-(4.4) with uniformity in (4.2), (4.4). Moreover, if we represent $\Lambda=\Lambda_{\Psi_{t}}$ outside a bounded set, then we assume that for every $N \geq 0$,

$$
\nabla_{t}^{N} \Psi_{t}^{\prime \prime} \in C_{b}^{\infty} \text {, uniformly in } t .
$$

We will write $p_{\Lambda}$ or sometimes only $p$ for the restriction $p_{\left.\right|_{\Lambda}}$. The zeros of $p$ in $\Lambda_{\Phi_{0}}+i \widetilde{W}$ are confined to a bounded set and there exists $m_{0} \in \mathbf{N}$, such that for every $\rho \in \Lambda_{\Phi_{0}}+i \widetilde{W}$, there exists $m \in\left\{0,1, \ldots, m_{0}\right\}$, such that $\nabla^{m} p(\rho) \neq 0$. If $\Lambda$ is an IR manifold as above, we conclude (since $\Lambda$ is maximally totally real) that for every $\rho \in \Lambda$, we have $\nabla^{m} p_{\Lambda}(\rho) \neq 0$ for some $0 \leq m \leq m_{0}$. Let $\rho_{0} \in \Lambda$ be a zero of $p_{\Lambda}$ and choose $m$ as above. Then we can find a real smooth vectorfield $\nu$ on neigh $\left(\rho_{0}, \Lambda\right)$ such that $\nu^{m} p_{\Lambda}\left(\rho_{0}\right) \neq 0$. Choose local coordinates $\left(x_{1}, x_{2}, . ., x_{2 n}\right)=\left(x_{1}, x^{\prime}\right)$ for $\Lambda$ centered at $\rho_{0}$, such that $\nu=\frac{\partial}{\partial x_{1}}$. By Malgrange's preparation theorem ([Ma]), we have in a neighborhood of $x=0$ :

$$
p_{\Lambda}(x)=q(x)\left(x_{1}^{m}+\sum_{1}^{m} a_{j}\left(x^{\prime}\right) x_{1}^{m-j}\right)=q(x) \prod_{j=1}^{m}\left(x_{1}-\lambda_{j}\left(x^{\prime}\right)\right),
$$

where $a_{j}\left(x^{\prime}\right), q(x)$ are smooth with $a_{j}(0)=0, q(0) \neq 0$, and $\lambda_{j}\left(x^{\prime}\right)$ are the zeros of the second factor in the middle expression. (The ordering of the roots does not matter.) If we let $\Lambda=\Lambda_{t}$ depend smoothly on $t \in$ neigh $\left(0, \mathbf{R}^{k}\right)$ as above, and let $\rho_{0}$ be a zero of $p_{\Lambda_{0}}$, and if we choose $x_{1}, . ., x_{2 n}$ to be local coordinates on $\Lambda_{t}$ depending smoothly on $t$ and centered at $\rho_{0}$ when $t=0$, then (4.6) extends to $p_{\Lambda_{t}}$ with $q=q(t, x), a_{j}=a_{j}\left(t, x^{\prime}\right)$ being smooth in $(t, x)$, and with $\lambda_{j}=\lambda_{j}\left(t, x^{\prime}\right)$. From (4.6), we get easily:

LEMMA 4.1. For the choice of coordinates above and for $\epsilon_{0}>0$ small enough, we have

$$
\lambda\left(\left\{x_{1} \in\left[-\epsilon_{0}, \epsilon_{0}\right] ;\left|p_{\Lambda}(x)\right| \leq \delta\right\}\right) \leq C \delta^{1 / m},
$$

for $\left|x^{\prime}\right| \leq \epsilon_{0}$ and $0<\delta \leq 1$. Here $\lambda$ denotes the one dimensional Lebesgue measure. In the parameter dependent case, the estimate is uniform in $t$ for $|t| \leq \epsilon_{0}$ (small enough).

Proof. It suffices to observe that $\left\{x_{1} \in\left[-\epsilon_{0}, \epsilon_{0}\right] ;\left|p_{\Lambda}(x)\right| \leq \delta\right\}$ is contained in the union of the intervals $\left[\operatorname{Re} \lambda_{j}\left(x^{\prime}\right)-C \delta^{1 / m}, \operatorname{Re} \lambda_{j}\left(x^{\prime}\right)+C \delta^{1 / m}\right]$.

If $\mu(d \rho)=\mu_{\Lambda}(d \rho)$ denotes the symplectic volume element on $\Lambda$, it follows that

$$
\mu\left(\left\{\rho \in \Lambda ;\left|p_{\Lambda}(\rho)\right| \leq \delta\right\}\right) \leq C \delta^{1 / m_{0}}
$$

again locally uniformly in $t$ in the parameter dependent case.

For $0<\epsilon \leq 1$, we put

$$
I_{\epsilon}(\Lambda, p)=\frac{1}{2} \int_{\Lambda} \log \frac{\left|p_{\Lambda}(\rho)\right|^{2}+\epsilon^{2}}{1+\epsilon^{2}} \mu(d \rho) .
$$


Notice that the integral converges in view of (4.1), (4.4). We also define $I(\Lambda, p)=$ $I_{0}(\Lambda, p)$ :

$$
I(\Lambda, p)=\int_{\Lambda} \log \left|p_{\Lambda}(\rho)\right| \mu(d \rho) .
$$

Here the convergence over a neighborhood of infinity follows from (4.1), (4.4) but the possible presence of zeros of $p_{\Lambda}$ requires a verification of the convergence of

$$
\int_{\left|p_{\Lambda}\right|^{-1}\left(\left[0, \frac{1}{2}\right]\right)} \log \left|p_{\Lambda}(\rho)\right| \mu(d \rho)=\int_{0}^{1 / 2} \log t d V(t),
$$

where $V(t)=\mu\left(\left|p_{\Lambda}\right|^{-1}([0, t])\right)=\mathcal{O}\left(t^{1 / m_{0}}\right)$, by (4.8). Integration by parts shows that the last integral is equal to

$$
[(\log t) V(t)]_{0}^{1 / 2}-\int_{0}^{1 / 2} \frac{1}{t} V(t) d t .
$$

From the dominated convergence theorem, we see that

$$
I_{\epsilon}(\Lambda, p) \rightarrow I(\Lambda, p), \epsilon \rightarrow 0
$$

Using (4.8), we can estimate the rate of convergence.

LEMma 4.2. We have

$$
I_{\epsilon}(\Lambda, p)-I(\Lambda, p)=\mathcal{O}\left(\epsilon^{1 / m_{0}}\right), \epsilon \rightarrow 0
$$

Proof. For $\epsilon>0$, we have

$$
\partial_{\epsilon} I_{\epsilon}(\Lambda, p)=\int_{\Lambda}\left[\frac{\epsilon}{\left|p_{\Lambda}(\rho)\right|^{2}+\epsilon^{2}}-\frac{\epsilon}{1+\epsilon^{2}}\right] \mu(d \rho) .
$$

The contribution to the integral from the region where $\left|p_{\Lambda}(\rho)\right|>1 / 2$ is $\mathcal{O}(\epsilon)$ and the contribution from the bounded region where $\left|p_{\Lambda}(\rho)\right| \leq 1 / 2$ is

$$
\begin{aligned}
& \mathcal{O}(\epsilon)+\int_{\left|p_{\Lambda}\right|^{-1}\left(\left[0, \frac{1}{2}\right]\right)} \frac{\epsilon}{\left(\epsilon^{2}+\left|p_{\Lambda}(\rho)\right|^{2}\right)} \mu(d \rho)=\mathcal{O}(\epsilon)+\int_{0}^{1 / 2} \frac{\epsilon}{\epsilon^{2}+t^{2}} d V(t)= \\
& \mathcal{O}(\epsilon)+\left[\frac{\epsilon}{\epsilon^{2}+t^{2}} V(t)\right]_{0}^{1 / 2}+\int_{0}^{1 / 2} \frac{2 \epsilon t}{\left(\epsilon^{2}+t^{2}\right)^{2}} V(t) d t= \\
& \mathcal{O}(\epsilon)+\mathcal{O}(1) \int_{0}^{1 / 2} \frac{2 t \epsilon}{\left(\epsilon^{2}+t^{2}\right)^{2}} t^{1 / m_{0}} d t=\mathcal{O}\left(\epsilon^{\frac{1}{m_{0}}-1}\right) .
\end{aligned}
$$

Hence $\partial_{\epsilon} I_{\epsilon}(\Lambda, p)=\mathcal{O}\left(\epsilon^{-1+1 / m_{0}}\right)$ and the Lemma follows by integration.

We next study the differential of $I_{\epsilon}$ and $I$ with respect to $\Lambda$. Let neigh $(0, \mathbf{R}) \ni$ $t \mapsto \Lambda_{t}$ be a smooth family of IR-manifolds as above, satisfying (4.1)-(4.5). We then have a corresponding generator $q_{t} \in C_{b}^{\infty}\left(\Lambda_{t} ; \mathbf{R}\right)$ such that a deformation field of the family is given by

$$
\nu_{t}=H_{q_{t}}^{\operatorname{Im} \sigma},
$$


when $\widetilde{q}_{t}$ is a smooth real extension of $q_{t}$ to a full neighborhood of $\Lambda_{t}$. If $f_{t}$ denotes an almost holomorphic extension of $q_{t}$, we take $\widetilde{q}_{t}=\operatorname{Re} f_{t}$, so that (cf. (1.19)):

$$
\nu_{t}=\widehat{i H_{f_{t}}} \text {, on } \Lambda_{t} \text {. }
$$

With

$$
F_{\epsilon}(p)=\frac{1}{2} \log \left(\frac{p \bar{p}+\epsilon^{2}}{1+\epsilon^{2}}\right)
$$

we have

$$
I_{\epsilon}\left(\Lambda_{t}, p\right)=\int_{\Lambda_{t}} F_{\epsilon}(p) \mu(d \rho)
$$

and

$$
\partial_{t} I_{\epsilon}\left(\Lambda_{t}, p\right)=\int_{\Lambda_{t}}\left(\frac{\partial F_{\epsilon}}{\partial p} \nu_{t}(p)+\frac{\partial F_{\epsilon}}{\partial \bar{p}} \overline{\nu_{t}(p)}\right) \mu(d \rho),
$$

where we also used that $\nu_{t}$ in (4.16) induces canonical transformations $\kappa_{t, s}: \Lambda_{s} \rightarrow \Lambda_{t}$, which conserve the symplectic volume element (see (1.19)). Since $p$ is holomorphic, we have $\nu_{t}(p)=i H_{f_{t}} p$, where in the last expression, we may view $H_{f_{t}}=\widehat{H_{f_{t}}}$ as the real Hamilton field on $\Lambda_{t}$ of $f_{\left.t\right|_{\Lambda_{t}}}=q_{t}$ with respect to $\sigma_{\left.\right|_{\Lambda_{t}}}$. From (4.17), we get

$$
\begin{aligned}
& \partial_{t} I_{\epsilon}\left(\Lambda_{t}, p\right)=\int_{\Lambda_{t}}\left(\frac{\partial F_{\epsilon}}{\partial p}(p) i H_{f_{t}} p-\frac{\partial F_{\epsilon}}{\partial \bar{p}}(p) i H_{f_{t}} \bar{p}\right) \mu(d \rho) \\
& =\frac{i}{2} \int_{\Lambda_{t}}\left\langle\frac{\bar{p}}{p \bar{p}+\epsilon^{2}} d p-\frac{p}{p \bar{p}+\epsilon^{2}} d \bar{p}, H_{f_{t}}\right\rangle \mu(d \rho)=\frac{i}{2} \int_{\Lambda_{t}}\left\langle\frac{p \bar{p}}{p \bar{p}+\epsilon^{2}}\left(\frac{d p}{p}-\frac{d \bar{p}}{\bar{p}}\right), H_{f_{t}}\right\rangle \mu(d \rho) .
\end{aligned}
$$

Here

$$
\frac{1}{2 i}\left(\frac{d p}{p}-\frac{d \bar{p}}{\bar{p}}\right)=d \arg p
$$

where $p=p_{\Lambda} \neq 0$, so we get

$$
\partial_{t} I_{\epsilon}\left(\Lambda_{t}, p\right)=-\int_{\Lambda_{t}}\left\langle\frac{p \bar{p}}{p \bar{p}+\epsilon^{2}} d\left(\arg p_{\Lambda_{t}}\right), H_{f_{t}}\right\rangle \mu(d \rho),
$$

which can also be written

$$
\partial_{t} I_{\epsilon}\left(\Lambda_{t}, p\right)=\int_{\Lambda_{t}}\left\langle\frac{p \bar{p}}{p \bar{p}+\epsilon^{2}} H_{\arg p_{\Lambda_{t}}}, d f_{t}\right\rangle \mu(d \rho)
$$

In the last two integrals the integration is restricted to $\Lambda_{t} \backslash p^{-1}(0)$, and the Hamilton fields are the ones for the real symplectic structure on $\Lambda_{t}$.

Proposition 4.3. The coefficients of the differential form $d \arg p_{\Lambda_{t}}$ (defined on $\left.\Lambda_{t} \backslash p^{-1}(0)\right)$ belong to $L^{1}\left(\Lambda_{t}, \mu\right)$.

Proof. The integrability near infinity follows from (4.1) (and we there identify $\Lambda_{t}$ with a neighborhood of infinity in $\mathbf{C}^{n}$ as indicated after (4.4)), so we only have to establish the local integrability near every point in $p^{-1}(0) \cap \Lambda_{t}$. Let $\rho_{0}$ be such a point and let $m \in\{1,2, .$.$\} be the order of vanishing, so that \nabla^{k} p\left(\rho_{0}\right)=0,0 \leq k \leq m-1$, $\nabla^{m} p\left(\rho_{0}\right) \neq 0$. Choose $2 n$ linearly independent and commuting vector fields $\nu_{1}, . ., \nu_{2 n}$ in a neighborhood of $\rho_{0}$ such that $\nu_{j}^{m} p\left(\rho_{0}\right) \neq 0$. Let $x_{1}, . ., x_{2 n}$ be the local coordinates centered at $\rho_{0}$ with $\nu_{j}=\frac{\partial}{\partial x_{j}}$. Let $A_{j}=\frac{\partial}{\partial x_{j}} \arg p_{\Lambda_{t}}$ for $x \in \operatorname{neigh}(0) \backslash p_{\Lambda_{t}}^{-1}(0)$, so that 
$d \arg p_{\Lambda_{t}}=\sum_{1}^{2 n} A_{j}(x) d x_{j}$. We have to show that $A_{j}$ is locally integrable near 0 and may choose $j=1$ for simplicity. Applying (4.6) to $p_{\Lambda}=p_{\Lambda_{t}}$, we see that

$$
\int_{\left\{x_{1} \in\left[-\epsilon_{0}, \epsilon_{0}\right] ; p_{\Lambda_{t}}(x) \neq 0\right\}}\left|\frac{\partial}{\partial x_{1}} \arg p_{\Lambda_{t}}(x)\right| d x_{1} \leq \text { Const. }
$$

for $\left|x^{\prime}\right| \leq \epsilon_{0}$, and the local integrability follows.

Let $[d \arg p]_{\Lambda_{t}}$ denote the differential form on $\Lambda_{t}$ with $L^{1}$ coefficients whose restriction to $\Lambda_{t} \backslash p^{-1}(0)$ is equal to $d \arg p_{\Lambda_{t}}$. Similarly let $\left[H_{\arg p}\right]_{\Lambda_{t}}$ be the $L^{1}$ vector field on $\Lambda_{t}$ determined by $H_{\arg p_{\Lambda_{t}}}$ on $\Lambda_{t} \backslash p^{-1}(0)$. Write

$$
\begin{gathered}
\partial_{t} I_{\epsilon}\left(\Lambda_{t}, p\right)=-\int_{\Lambda_{t}}\left\langle\frac{p \bar{p}}{p \bar{p}+\epsilon^{2}}[d \arg p]_{\Lambda_{t}}, H_{f_{t}}\right\rangle \mu(d \rho), \\
\partial_{t} I_{\epsilon}\left(\Lambda_{t}, p\right)=\int_{\Lambda_{t}}\left\langle\frac{p \bar{p}}{p \bar{p}+\epsilon^{2}}\left[H_{\arg p}\right]_{\Lambda_{t}}, d f_{t}\right\rangle \mu(d \rho) .
\end{gathered}
$$

$H_{f_{t}}, d f_{t}$ have bounded coefficients near infinity (using the identification with $\mathbf{C}^{n}$ near infinity indicated after (4.4)), and from Proposition 4.3 and the dominated convergence theorem, we get the first part of the following result:

Proposition 4.4. We have $\partial_{t} I_{\epsilon}\left(\Lambda_{t}, p\right) \rightarrow " \partial_{t} I\left(\Lambda_{t}, p\right) ", \epsilon \rightarrow 0$, where

$$
" \partial_{t} I\left(\Lambda_{t}, p\right) "=-\int_{\Lambda_{t}}\left\langle[d \arg p]_{\Lambda_{t}}, H_{f_{t}}\right\rangle \mu(d \rho)=\int_{\Lambda_{t}}\left\langle\left[H_{\arg p}\right]_{\Lambda_{t}}, d f_{t}\right\rangle \mu(d \rho) .
$$

Moreover, $t \mapsto I\left(\Lambda_{t}, p\right)$ is a Lipschitz function and the a.e. derivative is given by $" \partial_{t} I\left(\Lambda_{t}, p\right) "$.

Here the last statement follows from the fact that $t \mapsto\left\|[d \arg p]_{\Lambda_{t}}\right\|_{L^{1}}$ is locally bounded, as we can see by adding a smooth $t$ dependence in (4.6).

A Hamilton field is divergence free in symplectic coordinates, so $\operatorname{div}\left[H_{\arg p}\right]_{\Lambda_{t}}$ is a distribution of order $\leq 1$ with support in $p^{-1}(0) \cap \Lambda_{t}$. From (4.24), we get

$$
" \partial_{t}\left(\Lambda_{t}, p\right) "=-\int_{\Lambda_{t}} f_{t} \operatorname{div}\left[H_{\arg p}\right]_{\Lambda_{t}} \mu(d \rho) .
$$

Notice that the integral does not change if we add a $t$-dependent constant to $f_{t}$. When $d p_{\Lambda}, \overline{d p_{\Lambda}}$ are pointwise linearly independent on $p_{\Lambda}^{-1}(0)$, we shall obtain in section 8 a simple expression for this distribution and see that it is a Radon measure.

5. Second derivative under non-autonomous flows. Let $I \ni t \mapsto \Lambda_{t}$ be a smooth deformation of IR-manifolds as in the preceding section, where $I$ is a small open interval which contains 0 . Let $f_{t} \in C_{b}^{\infty}\left(\Lambda_{t} ; \mathbf{R}\right)$ be a corresponding smooth family of generating functions (unique up to a $t$ dependent constant) so that if we extend $f_{t}$ to an almost holomorphic function in a neighborhood of $\Lambda_{t}$, then a corresponding deformation field is given at $\Lambda_{t}$ by

$$
\nu_{t}=i \widehat{H_{f_{t}}}=2 \operatorname{Re}\left(i H_{f_{t}}\right),
$$

and we recall that $\nu_{t}$ generates a family $\kappa_{t, s}: \Lambda_{s} \rightarrow \Lambda_{t}$ of canonical diffeomorphisms. 
Let $p$ be a holomorphic function as in the preceding section and let $F$ be a smooth real-valued function defined on a complex neighborhood of $\overline{p\left(\Lambda_{I}\right)}$, with $F(1)=0$, where $\Lambda_{I}=\cup_{t \in I} \Lambda_{t}$. Put

$$
J(t)=\int_{\Lambda_{t}} F(p) \mu(d \rho) .
$$

With $p_{t}=p \circ \kappa_{t}, \kappa_{t}=\kappa_{t, 0}$, we get

$$
J(t)=\int_{\Lambda_{0}} F\left(p_{t}\right) \mu(d \rho)
$$

and

$$
\begin{aligned}
\partial_{t} J(t) & =\int_{\Lambda_{0}}\left[\frac{\partial F}{\partial p}\left(p_{t}\right) \partial_{t} p_{t}+\frac{\partial F}{\partial \bar{p}}\left(p_{t}\right) \overline{\partial_{t} p_{t}}\right] \mu(d \rho) \\
& =\int_{\Lambda_{t}}\left[\frac{\partial F}{\partial p}(p) i H_{f_{t}} p-\frac{\partial F}{\partial \bar{p}} i \overline{H_{f_{t}} p}\right] \mu(d \rho) .
\end{aligned}
$$

Since $p$ is holomorphic near $\Lambda_{t}$, we can consider (as in the preceding section) $H_{f_{t}}$ as the real Hamilton field $H_{f_{t}}^{\sigma_{\Lambda_{t}}}$. Viewing in the same way $H_{p}, H_{\bar{p}}$ as complex Hamilton fields on $\Lambda_{t}$, we get

$$
\begin{aligned}
\partial_{t} J(t) & =i \int_{\Lambda_{t}}\left[\frac{\partial F}{\partial p}(p) H_{f_{t}}(p)-\frac{\partial F}{\partial \bar{p}}(p) H_{f_{t}}(\bar{p})\right] \mu(d \rho) \\
& =\frac{1}{i} \int_{\Lambda_{t}}\left[\frac{\partial F}{\partial p}(p) H_{p}\left(f_{t}\right)-\frac{\partial F}{\partial \bar{p}}(p) H_{\bar{p}}\left(f_{t}\right)\right] \mu(d \rho) .
\end{aligned}
$$

Since the transpose of $H_{p}$ with respect to $\mu(d \rho)$ is equal to $-H_{p}$, we get

$$
\partial_{t} J(t)=\int_{\Lambda_{t}} G_{t}(\rho) f_{t}(\rho) \mu(d \rho), G_{t}(\rho)=i\left(H_{p}\left(\frac{\partial F}{\partial p}\right)-H_{\bar{p}}\left(\frac{\partial F}{\partial \bar{p}}\right)\right) .
$$

Notice that $G_{t}(\rho)$ is defined on $\Lambda_{t}$ with $H_{p}, H_{\bar{p}}$ defined as complex vectorfields on $\Lambda_{t}$, so $G_{t}(\rho)$ depends both on $\rho$ and on $T_{\rho} \Lambda_{t}$.

Let $I \ni t \mapsto \widetilde{\Lambda}_{t}$ be a second family with the same properties and let $\widetilde{f}_{t}$ be a corresponding smooth family of generating fucntions, that we also extend almost holomorphically. We assume that

$$
\begin{gathered}
\widetilde{\Lambda}_{0}=\Lambda_{0}, \\
\widetilde{f}_{0}=f_{0} .
\end{gathered}
$$

Equivalently, we have

$$
\operatorname{dist}\left(\widetilde{\Lambda}_{t}, \Lambda_{t}\right)=\mathcal{O}\left(t^{2}\right)
$$

Possibly after shrinking $I$ around 0 , we can define

$$
\widetilde{J}(t)=\int_{\widetilde{\Lambda}_{t}} F(p) \mu(d \rho),
$$

and analogously to (5.3), we have

$$
\partial_{t} \widetilde{J}(t)=\int_{\widetilde{\Lambda}_{t}} \widetilde{G}_{t}(\rho) \widetilde{f}_{t}(\rho) \mu(d \rho) .
$$


Let $\Phi_{t}=\widetilde{\kappa}_{t} \circ \kappa_{t}^{-1}: \Lambda_{t} \rightarrow \widetilde{\Lambda}_{t}$, where $\widetilde{\Lambda}_{t}=\widetilde{\kappa}_{t}\left(\Lambda_{0}\right)$, so that $\Phi_{t}$ is symplectic and

$$
\Phi_{t}(\rho)-\rho=\mathcal{O}\left(t^{2}\right)
$$

Since we also have dist $\left(T_{\Phi_{t}(\rho)}\left(\widetilde{\Lambda}_{t}\right), T_{\rho}\left(\Lambda_{t}\right)\right)=\mathcal{O}\left(t^{2}\right)$, we get $\widetilde{G}_{t}\left(\Phi_{t}(\rho)\right)=G_{t}(\rho)+\mathcal{O}\left(t^{2}\right)$, and hence

$$
\partial_{t} \widetilde{J}(t)-\partial_{t} J(t)=\int_{\Lambda_{t}} G_{t}(\rho) g_{t}(\rho) \mu(d \rho)+\mathcal{O}\left(t^{2}\right)
$$

where

$$
g_{t}=\tilde{f}_{t} \circ \Phi_{t}-f_{t}
$$

and we can assume that $g_{t}(\rho) \in C^{\infty}\left(I \times \operatorname{neigh}\left(\Lambda_{0}\right)\right)$ with $g_{t}(\cdot)$ almost holomorphic on $\Lambda_{t}$. We can also assume that

$$
g_{0}(\rho) \equiv 0
$$

(5.10) implies that

$$
\begin{aligned}
& \partial_{t}^{2} \widetilde{J}(0)-\partial_{t}^{2} J(0)=\int_{\Lambda_{0}} G_{0}(\rho)\left(\partial_{t} g\right)_{t=0}(\rho) \mu(d \rho) \\
& =i \int_{\Lambda_{0}}\left[H_{p}\left(\frac{\partial F}{\partial p}\right)-H_{\bar{p}}\left(\frac{\partial F}{\partial \bar{p}}\right)\right]\left(\left(\partial_{t} \widetilde{f}_{t}\right)_{t=0}-\left(\partial_{t} f_{t}\right)_{t=0}\right)(\rho) \mu(d \rho),
\end{aligned}
$$

and where we used (5.9) in the last step. Here we can undo the previous integration by parts and get

$$
\partial_{t}^{2} \widetilde{J}(0)-\partial_{t}^{2} J(0)=\frac{1}{i} \int_{\Lambda_{0}}\left[\frac{\partial F}{\partial p} H_{p}\left(\partial_{t} \widetilde{f}-\partial_{t} f\right)_{t=0}-\frac{\partial F}{\partial \bar{p}} H_{\bar{p}}\left(\partial_{t} \widetilde{f}-\partial_{t} f\right)_{t=0}\right] \mu(d \rho) .
$$

Notice that $\left(\partial_{t} f_{t}\right)_{t=0}=\partial_{t}\left(f\left(t, \kappa_{t}(\rho)\right)\right)_{t=0}$ is real, and similarly for $\partial_{t} \widetilde{f}_{t}$.

In order to exploit the last relation, we need to compute the second derivative in the case of "autonomous flows", and we shall temporarily consider the case of a special family of deformations. Let $\tilde{f} \in C_{b}^{\infty}\left(\Lambda_{0} ; \mathbf{R}\right)$ and denote by $\widetilde{f}$ also an almost holomorphic extension of $\widetilde{f}$. For $t \in$ neigh $(0, \mathbf{C})$, consider the IR-manifolds $\widetilde{\Lambda}_{t}=$ $\widetilde{\kappa}_{t}\left(\Lambda_{0}\right)$, where $\widetilde{\kappa}_{t}=\exp H_{\operatorname{Re}(t \widetilde{f})}^{\operatorname{Im} \sigma}$. To infinite order on $\Lambda_{0}$, we have

$$
H_{\operatorname{Re}(t \widetilde{f})}^{\operatorname{Im} \sigma} \equiv \widehat{t t H}_{\widetilde{f}} \equiv-H_{\operatorname{Im}(t \widetilde{f})}^{\operatorname{Re} \sigma}
$$

and it follows that $\widetilde{\kappa}_{t}^{*} \sigma=\sigma+\mathcal{O}\left(t^{\infty}\right)$ on $\Lambda_{0}$. Let $\partial_{t}, \partial_{\bar{t}}$ denote holomorphic and antiholomorphic derivatives. We also see that $\partial_{\bar{t}} \widetilde{\kappa}_{t}(\rho)=\mathcal{O}\left(t^{\infty}\right)$ on $\Lambda_{0}$. We have

$$
\begin{gathered}
\widetilde{J}(t):=\int_{\widetilde{\Lambda}_{t}} F(p) \mu(d \rho)=\int_{\Lambda_{0}} F\left(p_{t}\right) \mu(d \rho)+\mathcal{O}\left(t^{\infty}\right), p_{t}:=p \circ \widetilde{\kappa}_{t} \\
\partial_{t} \widetilde{J}(t)=\int_{\Lambda_{0}} \partial_{t}\left(F\left(p_{t}\right)\right) \mu(d \rho)+\mathcal{O}\left(t^{\infty}\right)= \\
\int_{\Lambda_{0}}\left(\frac{\partial F}{\partial p}\left(p_{t}\right) \partial_{t} p_{t}+\frac{\partial F}{\partial \bar{p}}\left(p_{t}\right) \overline{\partial_{\bar{t}} p_{t}}\right) \mu(d \rho)+\mathcal{O}\left(t^{\infty}\right)=\int_{\Lambda_{0}} \frac{\partial F}{\partial p}\left(p_{t}\right) \partial_{t} p_{t} \mu(d \rho)+\mathcal{O}\left(t^{\infty}\right) \\
\partial_{\bar{t}} \partial_{t} \widetilde{J}(t)=\int_{\Lambda_{0}} \frac{\partial^{2} F}{\partial \bar{p} \partial p}\left(p_{t}\right) \overline{\partial_{t} p_{t}} \partial_{t} p_{t} \mu(d \rho)+\mathcal{O}\left(t^{\infty}\right) .
\end{gathered}
$$


For $t=0$ we have $\partial_{t} p_{t}=i H_{\widetilde{f}}(p)$ and hence

$$
\left(\partial_{\bar{t}} \partial_{t} \widetilde{J}(t)\right)_{t=0}=\int_{\Lambda_{0}} \frac{\partial^{2} F}{\partial \bar{p} \partial p}(p)\left(H_{\widetilde{f}} p\right)\left(\overline{H_{\widetilde{f}}(p)}\right) \mu(d \rho) .
$$

Next we compute

$$
\begin{aligned}
\partial_{\operatorname{Im} t} \widetilde{J}(t) & =i\left(\partial_{t} \widetilde{J}(t)-\partial_{\bar{t}} \widetilde{J}(t)\right)=i \int_{\widetilde{\Lambda}_{0}}\left(\frac{\partial F}{\partial p}\left(p_{t}\right) \partial_{t} p_{t}-\frac{\partial F}{\partial \bar{p}}\left(p_{t}\right) \overline{\partial_{t} p_{t}}\right) \mu(d \rho)+\mathcal{O}\left(t^{\infty}\right) \\
& =i \int_{\widetilde{\Lambda}_{t}}\left(\frac{\partial F}{\partial p}(p) i H_{\widetilde{f}} p-\frac{\partial F}{\partial \bar{p}}(p) \overline{i H_{\widetilde{f}} p}\right) \mu(d \rho)+\mathcal{O}\left(t^{\infty}\right)
\end{aligned}
$$

Modulo $\mathcal{O}\left(t^{\infty}\right)$, we have $H_{\widetilde{f}} p=H_{\widetilde{f}}^{\sigma_{\tilde{\Lambda}_{t}}} p, \operatorname{Im} \tilde{f}=0$, on $\widetilde{\Lambda}_{t}$, so we get

$$
\begin{aligned}
\partial_{\operatorname{Im} t} \widetilde{J}(t)= & -\int_{\widetilde{\Lambda}_{t}}\left(\frac{\partial F}{\partial p}(p) H_{\operatorname{Re} \tilde{f}}^{\sigma_{\mid \tilde{I}_{t}}} p+\frac{\partial F}{\partial \bar{p}} H_{\operatorname{Re} \tilde{f}}^{\sigma_{\mid \tilde{I}_{t}}} \bar{p}\right) \mu(d \rho)+\mathcal{O}\left(t^{\infty}\right) \\
& =-\int_{\widetilde{\Lambda}_{t}} H_{\operatorname{Re} \tilde{f}}^{\sigma_{\mid \tilde{\Lambda}_{t}}}(F(p)) \mu(d \rho)+\mathcal{O}\left(t^{\infty}\right)=\mathcal{O}\left(t^{\infty}\right) .
\end{aligned}
$$

(The idea behind this is that in the case when $\widetilde{f}$ is holomorphic, then $\widetilde{\Lambda}_{t}=\exp \widehat{i t H}_{\tilde{f}}\left(\Lambda_{0}\right)$ only depends on Ret, and hence we have the same for $\widetilde{J}(t)$.) The last relation can be differentiated any number of times w.r.t. $\operatorname{Re} t, \operatorname{Im} t$ and combining this with (5.15), we get

$$
\left(\partial_{\operatorname{Re} t}^{2} \widetilde{J}(t)\right)_{t=0}=4\left(\partial_{\bar{t}} \partial_{t} \widetilde{J}(t)\right)_{t=0}=4 \int_{\Lambda_{0}} \frac{\partial^{2} F}{\partial \bar{p} \partial p}(p) H_{\tilde{f}}(p) \overline{H_{\tilde{f}}(p)} \mu(d \rho) .
$$

We now restrict the attention to real $t$ and write $\partial_{t}$ instead of $\partial_{\operatorname{Re}} t$. In order to apply (5.17) in (5.13), we let $\widetilde{f}(t, \rho) \in C_{b}^{\infty}\left(I \times \mathbf{C}^{2 n}\right)$ be such that $\widetilde{f}(t, \cdot)$ is an almost holomorphic extension of $\widetilde{f}_{\mid \tilde{\Lambda}_{t}}$ and observe that $\partial_{t} \widetilde{f}(t, \rho)=\mathcal{O}\left(t^{\infty}+\operatorname{dist}\left(\rho, \Lambda_{0}\right)^{\infty}\right)$. We can apply (5.13) and get the following identity for $t=0$ :

$$
\begin{aligned}
\partial_{t}^{2} J(t)=4 \int_{\Lambda_{t}} \frac{\partial^{2} F}{\partial \bar{p} \partial p} H_{f_{t}}(p) H_{f_{t}}(\bar{p}) & \mu(d \rho) \\
& +i \int_{\Lambda_{t}}\left[H_{p}\left(\frac{\partial F}{\partial p}\right)-H_{\bar{p}}\left(\frac{\partial F}{\partial \bar{p}}\right)\right] \partial_{t} f_{t} \mu(d \rho) .
\end{aligned}
$$

Comparing with (5.3), we see that the last term in (5.18) is equal to $\left(\partial_{s}\right)_{s=0} J(t, s)$, with $J(t, s)=\int_{\Lambda_{t, s}} F(p) \mu(d \rho), \Lambda_{t, s}=\exp s 2 \operatorname{Re}\left(i H_{\partial_{t} f_{t}}\right)\left(\Lambda_{t}\right)$. Clearly this extends to general $t$ and the last term can also be written

$$
\frac{1}{i} \int_{\Lambda_{t}}\left(\frac{\partial F}{\partial p} H_{p}-\frac{\partial F}{\partial \bar{p}} H_{\bar{p}}\right) \partial_{t} f_{t} \mu(d \rho),
$$

where $H_{p}, H_{\bar{p}}$ in the last two formulae denote the complex vector fields on $\Lambda_{t}$.

Notice that the bracket in the last integral in (5.18) can also be written as

$$
H_{p}\left(\frac{\partial F}{\partial p}\right)-H_{\bar{p}}\left(\frac{\partial F}{\partial \bar{p}}\right)=2 \frac{\partial^{2} F}{\partial p \partial \bar{p}}\{p, \bar{p}\}
$$


where $\{p, \bar{p}\}=H_{p}(\bar{p})$ denotes the Poisson bracket of $p$ and $\bar{p}$ for the symplectic structure of $\Lambda_{t}$.

6. Continuity and convergence for the differential of $\Lambda \mapsto I(\Lambda, p)$. Let $\Lambda$ be an IR-manifold and $p$ a holomorphic function as in section 4 . We recall (see also below) that by Malgrange's preparation theorem, the differential form $d \arg p$ on $\Lambda \backslash p^{-1}(0)$ has $L^{1}$ coefficients, and the same holds for the Hamilton field $H_{\arg p}$. Let $[d \arg p]_{\Lambda}$ and $\left[H_{\arg p}\right]_{\Lambda}$ denote the corresponding $L^{1}$ form and $L^{1}$ vectorfield respectively on $\Lambda$.

We review some calculations for the differential of $\Lambda \mapsto I(\Lambda, p)$. Let $t \mapsto \Lambda_{t}$ be a smooth deformation of IR-manifolds as in section 4 , where $I$ is an open bounded interval containing $t=0$, and let $t \mapsto f(t, \rho) \in C_{b}^{\infty}\left(\Lambda_{t} ; \mathbf{R}\right)$ be a corresponding generating family (unique up to a $t$ dependent constant and extended to be almost holomorphic in $\rho$ ), so that $\Lambda_{t}=\Phi_{t}\left(\Lambda_{0}\right)$, with $\partial_{t} \Phi_{t}(\rho)=\widehat{i H_{f_{t}}}\left(\Phi_{t}(\rho)\right), \rho \in \Lambda_{0}$. With $F_{\epsilon}(p)=\frac{1}{2} \log \left(\frac{p \bar{p}+\epsilon^{2}}{1+\epsilon^{2}}\right)$, we obtained in section 4 that

$$
I_{\epsilon}\left(\Lambda_{t}, p\right)=\int_{\Lambda_{t}} F_{\epsilon}(p) \mu(d \rho)=I\left(\Lambda_{t}, p\right)+\mathcal{O}\left(\epsilon^{1 / m}\right),
$$

for some $m \in \mathbf{N} \backslash\{0\}$. Moreover, we saw that

$$
\partial_{t} I_{\epsilon}\left(\Lambda_{t}, p\right)=\frac{i}{2} \int_{\Lambda_{t}}\left\langle\frac{p \bar{p}}{p \bar{p}+\epsilon^{2}}\left(\frac{d p}{p}-\frac{d \bar{p}}{\bar{p}}\right), H_{f_{t}}\right\rangle \mu(d \rho) .
$$

Here $\frac{1}{2 i}\left(\frac{d p}{p}-\frac{d \bar{p}}{\bar{p}}\right)=d \arg p$ where $p \neq 0$, so that

$$
\begin{aligned}
& \partial_{t} I_{\epsilon}\left(\Lambda_{t}, p\right)=-\int_{\Lambda_{t}}\left\langle\frac{p \bar{p}}{p \bar{p}+\epsilon^{2}}[d(\arg p)]_{\Lambda_{t}}, H_{f_{t}}\right\rangle \mu(d \rho) \\
& =\int_{\Lambda_{t}}\left\langle\frac{p \bar{p}}{p \bar{p}+\epsilon^{2}}\left[H_{\arg p}\right]_{\Lambda_{t}}, d f_{t}\right\rangle \mu(d \rho) .
\end{aligned}
$$

By the dominated convergence theorem, we got

$$
\partial_{t} I_{\epsilon}\left(\Lambda_{t}, p\right) \rightarrow-\int_{\Lambda_{t}}\left\langle[d(\arg p)]_{\Lambda_{t}}, H_{f_{t}}\right\rangle \mu(d \rho)=\int_{\Lambda_{t}}\left\langle\left[H_{\arg p}\right]_{\Lambda_{t}}, d f_{t}\right\rangle \mu(d \rho),
$$

when $\epsilon \rightarrow 0$, and since $\left\|[d(\arg p)]_{\Lambda_{t}}\right\|_{L^{1}(\Lambda, \mu)}$ is locally uniformly bounded with respect to $t$, we concluded that $t \mapsto I_{\epsilon}\left(\Lambda_{t}, p\right)$ is uniformly Lipschitz with respect to $\epsilon$, and in particular that $t \mapsto I\left(\Lambda_{t}, p\right)$ is Lipschitz and that the a.e. derivative of the last function is given by

$$
" \partial_{t} I\left(\Lambda_{t}, p\right) "=-\int_{\Lambda_{t}}\left\langle[d(\arg p)]_{\Lambda_{t}}, H_{f_{t}}\right\rangle \mu(d \rho)=\int_{\Lambda_{t}}\left\langle\left[H_{\arg p}\right]_{\Lambda_{t}}, d f_{t}\right\rangle \mu(d \rho) .
$$

In a more fancy way, we can consider an open connected set $L$ in the family of smooth IR-manifolds as in section 4 , and define the "distance" between $\Lambda_{0}, \Lambda_{1} \in L$ to be

$$
\operatorname{dist}\left(\Lambda_{0}, \Lambda_{1}\right)=\inf \int_{0}^{1}\left\|d f_{t}\right\|_{L^{\infty}\left(\Lambda_{t}\right)} \mu(d \rho) d t,
$$


where the infimum is taken over all smooth curves $[0,1] \ni t \mapsto \Lambda_{t} \in L$, that link $\Lambda_{0}$ to $\Lambda_{1}$. (We did not check that this is really a distance, nor did we consider the problem of studying the completion of $L$.) We can think of $L$ as a manifold. The tangent space of $L$ at a point $\Lambda$ is then the space of all $d f$, where $f \in C_{b}^{\infty}(\Lambda ; \mathbf{R})$. In the same spirit, we have the differentials

$$
\begin{gathered}
\left\langle d_{\Lambda} I_{\epsilon}(\Lambda, p), \delta \Lambda\right\rangle=\int_{\Lambda}\left\langle\frac{p \bar{p}}{p \bar{p}+\epsilon^{2}}\left[H_{\arg p}\right]_{\Lambda}, d f\right\rangle \mu(d \rho), \\
\left\langle " d_{\Lambda} I(\Lambda, p) ", \delta \Lambda\right\rangle=\int_{\Lambda}\left\langle\left[H_{\arg p}\right]_{\Lambda}, d f\right\rangle \mu(d \rho)=-\int_{\Lambda}\left\langle[d \arg p]_{\Lambda}, H_{f}\right\rangle \mu(d \rho),
\end{gathered}
$$

where $d f$ is the differential on $\Lambda$ corresponding to the infinitesimal variation $\delta \Lambda$. Here " $d_{\Lambda} I(\Lambda, p)$ " becomes the almost everywhere differential of our Lipschitz function $I(\cdot, p)$ whenever we restrict $\Lambda$ to vary in a curve or more generally in a finite dimensional submanifold.

We next give sufficient conditions for the continuity of " $d_{\Lambda} I(\cdot, p)$ " at some given point $\Lambda$, and for having a power law estimate in the convergence $d_{\Lambda} I_{\epsilon}(\Lambda, p) \rightarrow$ " $d_{\Lambda} I(\Lambda, p)$ ". We start with the continuity question. Let $\Lambda_{0}$ be a fixed IR-manifold (as in section 4). Let $x_{0} \in \Lambda_{0}$ be a point, where $p\left(x_{0}\right)=0$ and choose local coordinates $\left(x_{1}, . ., x_{2 n}\right)$ centered at $x_{0}$, such that for some $0<N_{0} \in \mathbf{N}: \partial_{x_{j}}^{N_{0}} p(0) \neq 0, \partial_{x_{j}}^{k} p(0)=0$, $0 \leq k \leq N_{0}-1,1 \leq j \leq 2 n$. Let $p_{\Lambda}=p_{\left.\right|_{\Lambda}}$. We are interested in studying how $d \arg p_{\Lambda}$ varies when we make a small variation of $\Lambda$. For simplicity, we concentrate on $\partial_{x_{1}} \arg p_{\Lambda}$, and we view $p_{\Lambda}$ as a small perturbation in $C^{\infty}$ of $p_{\Lambda_{0}}$, by choosing coordinates $\left(x_{1}, . ., x_{2 n}\right)$ on $\Lambda_{t}$ depending smoothly on $t$.

By Malgrange's preparation theorem:

$$
p_{\Lambda_{0}}(x)=q(x)\left(x_{1}^{N_{0}}+\sum_{1}^{N_{0}} a_{j}\left(x^{\prime}\right) x_{1}^{N_{0}-j}\right),
$$

where $q, a_{j}$ are smooth with $q(0) \neq 0, a_{j}(0)=0$, and we write $\left(x_{1}, x_{2}, . ., x_{2 n}\right)=$ $\left(x_{1}, x^{\prime}\right)$. Let $\lambda_{1}\left(x^{\prime}\right), . ., \lambda_{N_{0}}\left(x^{\prime}\right)$ be the roots of the last factor of (6.8) (where the ordering doesn't matter) so that locally, up to a multiple of $2 \pi$,

$$
\arg p_{\Lambda_{0}}(x)=\arg (q(x))+\sum_{1}^{N_{0}} \arg \left(x_{1}-\lambda_{j}\left(x^{\prime}\right)\right), \text { for } p_{\Lambda_{0}}(x) \neq 0 .
$$

From this we see that

$$
\int_{-\epsilon_{0}}^{\epsilon_{0}}\left|\partial_{x_{1}} \arg p_{\Lambda_{0}}(x)\right| d x_{1} \leq C<\infty
$$

for $\left|x^{\prime}\right| \leq \epsilon_{0}$, with $\epsilon_{0}>0$ fixed and sufficiently small, and where the integration is restricted to the points where $p_{\Lambda_{0}}(x) \neq 0$. Assume,

$$
\bigcup_{j=1}^{N_{0}}\left\{x^{\prime} \in \mathbf{R}^{2 n-1} ;\left|x^{\prime}\right| \leq \epsilon_{0}, \lambda_{j}\left(x^{\prime}\right) \in \mathbf{R}\right\} \text { is of Lebesgue measure } 0 . \quad\left(\mathrm{H}\left(x_{1}\right)\right)
$$


(Equivalently, $\Pi_{x^{\prime}}\left(p_{\Lambda_{0}}^{-1}(0) \cap\left(\left[-\epsilon_{0}, \epsilon_{0}\right] \times B\left(0, \epsilon_{0}\right)\right)\right)$ should be of measure 0 , where $\Pi_{x^{\prime}}$ denotes the projection $x \mapsto x^{\prime}$.) Let $\Lambda_{t}, t \in \operatorname{neigh}\left(0, \mathbf{R}^{k}\right)$ be a smooth family of IR-manifolds. Then for $t$ sufficiently close to $0,(6.8)$ extends:

$$
p_{\Lambda_{t}}(x)=q(x, t)\left(x_{1}^{N_{0}}+\sum_{1}^{N_{0}} a_{j}\left(x^{\prime}, t\right) x_{1}^{N_{0}-j}\right)
$$

(choosing the local coordinates to depend smoothly on $t$ ) and we want to estimate

$$
\int\left|\partial_{x_{1}} \arg p_{\Lambda_{0}}(x)-\partial_{x_{1}} \arg p_{\Lambda_{t}}(x)\right| d x
$$

where we integrate over $\left\{x ;\left|x_{1}\right|<\epsilon_{0},\left|x^{\prime}\right|<\epsilon_{0}, p_{\Lambda_{0}}(x) \neq 0 \neq p_{\Lambda_{t}}(x)\right\}$. The corresponding integral

$$
K_{t}\left(x^{\prime}\right)=\int\left|\partial_{x_{1}} \arg p_{\Lambda_{0}}(x)-\partial_{x_{1}} \arg p_{\Lambda_{t}}(x)\right| d x_{1}
$$

is a bounded function of $x^{\prime}$, and converges to 0 when $t \rightarrow 0$ for $x^{\prime}$ outside the set in $\left(\mathrm{H}\left(x_{1}\right)\right)$. So under that assumption, the integral (6.12) converges to 0 when $t \rightarrow 0$.

We next find an equivalent form of $\left(\mathrm{H}\left(x_{1}\right)\right)$, which is easier to formulate globally on $\Lambda_{0}$ : Introduce the assumption

$$
\begin{aligned}
& \text { For every smooth hypersurface } \Gamma \subset]-\epsilon_{0}, \epsilon_{0}\left[\times B\left(0, \epsilon_{0}\right),\right. \\
& p_{\Lambda_{0}}^{-1}(0) \cap \Gamma \text { is of (Lebesgue) measure } 0 \text { in } \Gamma \text {. }
\end{aligned}
$$

Proposition 6.1. (A) and $\left(H\left(x_{1}\right)\right)$ are equivalent.

Proof. We first prove $\left(\mathrm{H}\left(x_{1}\right)\right) \Rightarrow(\mathrm{A})$ and assume that $(\mathrm{A})$ does not hold. Let $\Gamma \subset]-\epsilon_{0}, \epsilon_{0}\left[\times B\left(0, \epsilon_{0}\right)\right.$ be a smooth hypersurface such that $p^{-1}(0) \cap \Gamma$ is of measure $>0$ in $\Gamma$. The set

$$
\left(p^{-1}(0) \cap \Gamma\right)_{\infty}=\left\{x \in \Gamma \cap p^{-1}(0) ; \frac{\operatorname{vol}\left(p^{-1}(0) \cap \Gamma \cap B(x, \epsilon)\right)}{\operatorname{vol}(\Gamma \cap B(x, \epsilon))} \rightarrow 1, \epsilon \rightarrow 0\right\}
$$

is of full measure in $\Gamma \cap p^{-1}(0)$ considered as a subset of $\Gamma$. We notice that $p_{\left.\right|_{\Gamma}}$ vanishes to infinite order on $\left(p^{-1}(0) \cap \Gamma\right)_{\infty}$. The set

$$
\begin{aligned}
F_{\infty}= & \left\{x \in\left(\Gamma \cap p^{-1}(0)\right)_{\infty} ;\right. \\
& \left.\frac{\operatorname{vol}\left(\left\{y \in\left(\Gamma \cap p^{-1}(0)\right)_{\infty} ;\left(d x_{2} \wedge . \wedge \wedge d x_{2 n}\right)_{\left.\right|_{\Gamma}}(y)=0\right\} \cap B(x, \epsilon)\right)}{\operatorname{vol}(B(x, \epsilon) \cap \Gamma)} \rightarrow 1, \epsilon \rightarrow 0\right\}
\end{aligned}
$$

is of full measure in $\left\{x \in \Gamma \cap p^{-1}(0) ;\left(d x_{2} \wedge . \wedge \wedge d x_{2 n}\right)_{\left.\right|_{\Gamma}}(x)=0\right\}$. At the points of $F_{\infty}$, the form $\left(d x_{2} \wedge . . \wedge d_{2 n}\right)_{\left.\right|_{\Gamma}}$ vanishes to infinite order as well as $p_{\left.\right|_{\Gamma}}$. Let $y^{0}=$ $\left(y_{1}^{0}, . ., y_{2 n}^{0}\right) \in F_{\infty}$. We may assume that $\Gamma$ is of the form $x_{2 n}=a\left(x_{1}, . ., x_{2 n-1}\right)$ near $y^{0}$. Then $\frac{\partial a}{\partial x_{1}}(x)$ vanishes to $\infty$ order at $\left(y_{1}^{0}, . ., y_{2 n-1}^{0}\right)$, so at $y^{0}$ the hypersurfaces $\Gamma$ and $x_{2 n}=a\left(y_{1}^{0}, x_{2}, . ., x_{2 n-1}\right)$ are tangent to $\infty$ order. It follows that $p\left(x_{1}, x_{2}, . ., x_{2 n-1}\right.$, $\left.a\left(y_{1}^{0}, x_{2}, . ., x_{2 n-1}\right)\right)$ vanishes to $\infty$ order at $\left(y_{1}^{0}, . ., y_{2 n-1}^{0}\right)$ in contradiction with the fact that $\partial_{x_{1}}^{N_{0}} p\left(y^{0}\right) \neq 0$. Consequently $F_{\infty}$ is empty and hence

$$
\left\{x \in \Gamma \cap p^{-1}(0) ;\left(d x_{2} \wedge . \wedge d_{2 n}\right)_{\left.\right|_{\Gamma}}(x)=0\right\}
$$


is of measure 0. In particular $\left\{x \in\left(p^{-1}(0) \cap \Gamma\right)_{\infty} ;\left(d x_{2} \wedge . . \wedge d x_{2 n}\right)_{\left.\right|_{\Gamma}}(x) \neq 0\right\}$ is of non-vanishing measure, and the same holds for its $x^{\prime}$ space projection, which is in contradiction with $\left(\mathrm{H}\left(x_{1}\right)\right)$. We have proved that $\left(\mathrm{H}\left(x_{1}\right)\right)$ implies $(\mathrm{A})$.

It remains to prove that (A) implies $\left(\mathrm{H}\left(x_{1}\right)\right)$. Recall that $\partial_{x_{1}}^{N_{0}} p \neq 0$ in ]$-\epsilon_{0}, \epsilon_{0}\left[\times B\left(0, \epsilon_{0}\right)\right.$. Without loss of generality, we may assume that $\partial_{x_{1}}^{N_{0}} \operatorname{Re} p \neq 0$ there. Then

$$
p^{-1}(0) \subset \cup_{N=1}^{N_{0}} \Gamma_{N}
$$

where

$$
\Gamma_{N}=\left\{x ; \partial_{x_{1}}^{N} \operatorname{Re} p(x) \neq 0, \partial_{x_{1}}^{N-1} \operatorname{Re} p(x)=0\right\}
$$

are smooth hypersurfaces such that $\left.\Pi_{x^{\prime}}\right|_{\Gamma_{j}}$ is a local diffeomorphism for $j=1, . ., N_{0}$. Applying (A) to $p^{-1}(0) \cap \Gamma_{j}$, it follows that $\Pi_{x^{\prime}}\left(p^{-1}(0)\right)$ is of measure 0 .

Combining Proposition 6.1 with its preceding discussion, we get

THEOREM 6.2. Let $\Lambda_{0}$ be an IR manifold and assume

$$
\begin{aligned}
& \text { For every smooth hypersurface } \Gamma \subset \Lambda_{0}, \\
& p^{-1}(0) \cap \Gamma \text { is of Lebesgue measure } 0 \text { in } \Gamma .
\end{aligned}
$$

Then " $d_{\Lambda} I(\cdot, p)$ " is continuous at $\Lambda=\Lambda_{0}$ in the following sense: Let neigh $\left(0, \mathbf{R}^{k}\right) \ni$ $t \mapsto \Lambda_{t}$ be a smooth family of IR manifolds as in section 4, such that $\Lambda_{t=0}=\Lambda_{0}$. Let $f_{1}(t), . ., f_{k}(t)$ be a corresponding system of generating functions on $\Lambda_{t}$, so that $f_{\ell}$ is generating for the one parameter family obtained by varying $t_{\ell}$, (freezing all the $t_{j}$ with $j \neq \ell$ ) and hence that

$$
" \partial_{t_{\ell}} I\left(\Lambda_{t}, p\right) "=-\int_{\Lambda_{t}}\left\langle[d \arg p]_{\Lambda_{t}}, H_{f_{\ell}(t)}\right\rangle \mu(d \rho) .
$$

Then " $\partial_{t_{\ell}} I\left(\Lambda_{t}, p\right)$ " are continuous at $t=0$ and consequently $t \mapsto I\left(\Lambda_{t}, p\right)$ is differentiable at that point with partial derivatives (without the ") given by (6.14).

In practice, it might be preferable to use Proposition 6.1, which says that ( $\left.\mathrm{A}_{\text {glob }}\right)$ holds iff for each $x_{0} \in p^{-1}(0)$ there is a (or equivalently for all) system(s) of smooth local coordinates $x_{1}, . ., x_{2 n}$ centered at that point, such that $\partial_{x_{1}}^{N_{0}} p(0) \neq 0$ for some $N_{0} \in \mathbf{N} \backslash\{0\}$ (that we choose to be minimal), and the roots $\lambda_{j}\left(x^{\prime}\right)$ of the last factor in (6.8) have imaginary parts that are $\neq 0$ on a set of full measure in a neighborhood of $x^{\prime}=0$.

We now turn to the question of having a power law for the convergence $d_{\Lambda} I_{\epsilon}\left(\Lambda_{0}, p\right)$ $\rightarrow " d_{\Lambda} I\left(\Lambda_{0}, p\right) ", \epsilon \rightarrow 0$. Here we did not find a nice invariant condition and content ourselves with

For every $x_{0} \in p^{-1}(0) \cap \Lambda_{0}$, we can find $2 n$ linearly independent vectorfields (B) $\nu_{1}, . ., \nu_{2 n}$ near $x_{0}$, such that $\nu_{j}^{k} p(0)=0,0 \leq k \leq N_{j}-1, \nu_{j}^{N_{j}} p(0) \neq 0$, for some $0<N_{j} \in \mathbf{N}$, and such that if $\Gamma_{j}$ is a hypersurface passing through $x_{0}$, transversal to $\nu_{j}$, then there exist $\epsilon_{0}, \delta_{0}>0$, such that for $0<\epsilon \leq \epsilon_{0}$, $\operatorname{vol}\left(\left\{x^{\prime} \in \Gamma_{j} ; \operatorname{dist}\left(x^{\prime}, x_{0}\right) \leq \epsilon_{0}, \inf _{|t|<\epsilon_{0}}\left|p\left(\exp t \nu_{j}\left(x^{\prime}\right)\right)\right|<\epsilon\right\}\right) \leq \epsilon^{\delta_{0}}$.

Proposition 6.3. Under the assumption (B), there exists $\delta_{1}>0$ such that

$$
[d \arg p]_{\Lambda_{0}}-\frac{p \bar{p}}{\epsilon^{2}+p \bar{p}}[d \arg p]_{\Lambda_{0}}=\mathcal{O}\left(\epsilon^{\delta_{1}}\right) \text {, in } L^{1} .
$$


Proof. The expression in (6.15) is equal to

$$
\frac{\epsilon^{2}}{p \bar{p}+\epsilon^{2}}[d \arg p]_{\Lambda_{0}} .
$$

The $L^{1}$ norm of this function over any region $|p| \geq$ Const. $>0$ is $\mathcal{O}\left(\epsilon^{2}\right)$, so we only have to examine what happens near a point $x_{0}$, where $p$ vanishes. If $\nu_{1}, . ., \nu_{2 n}$ are the corresponding vectorfields appearing in (B), it is enough to show that

$$
\left\|\frac{\epsilon^{2}}{p \bar{p}+\epsilon^{2}}\left[\nu_{j}(\arg p)\right]_{\Lambda_{0}}\right\|_{L^{1}\left(\operatorname{neigh}\left(x_{0}, \Lambda_{0}\right)\right)}=\mathcal{O}\left(\epsilon^{\delta_{1}}\right),
$$

for every $j$, with the obvious definition of $\left[\nu_{j}(\arg p)\right]_{\Lambda_{0}}$. Fix a $j$ and choose local coordinates $x_{1}, \ldots, x_{2 n}$ centered at $x_{0}$, such that $\nu_{j}=\partial_{x_{1}}$.

Independently of the proof we are engaged in, it may be of interest to notice that if we write (6.8) and let $\lambda_{1}\left(x^{\prime}\right), . ., \lambda_{N_{j}}\left(x^{\prime}\right)$ be the corresponding roots, (with $N_{j}=N_{0}$ ) then the volume estimate in (B) implies that for some new $\delta_{0}$ :

$$
\operatorname{vol}\left(\left\{x^{\prime} \in B\left(0, \epsilon_{0}\right) ;\left|\operatorname{Im} \lambda_{k}\left(x^{\prime}\right)\right| \leq \epsilon\right\}\right) \leq \mathcal{O}\left(\epsilon^{\delta_{0}}\right),
$$

for all $k$. Conversely we can go from (6.18) to the volume estimate in (B) (with a new $\left.\delta_{0}\right)$.

Rather than quoting (6.8) directly we shall only use the fact that

$$
\sup _{\left|x^{\prime}\right| \leq \epsilon_{0}} \int_{-\epsilon_{0}}^{\epsilon_{0}}\left|\partial_{x_{1}} \arg p(x)\right| d x_{1} \leq \text { Const. }
$$

Let $0<\epsilon \leq \widetilde{\epsilon} \ll 1$. Then, with $q\left(x^{\prime}\right)=\inf _{-\epsilon_{0}<t<\epsilon_{0}}\left|p\left(t, x^{\prime}\right)\right|$,

$$
\begin{aligned}
& \int_{B\left(0, \epsilon_{0}\right)} \int_{-\epsilon_{0}}^{\epsilon_{0}} \frac{\epsilon^{2}}{p \bar{p}+\epsilon^{2}}\left|\partial_{x_{1}} \arg p(x)\right| d x_{1} d x^{\prime}= \\
& \int_{q\left(x^{\prime}\right) \leq \widetilde{\epsilon}} \int\left|\partial_{x_{1}} \arg p(x)\right| d x_{1} d x^{\prime}+\mathcal{O}\left(\left(\frac{\epsilon}{\widetilde{\epsilon}}\right)^{2}\right) \leq \mathcal{O}\left(\widetilde{\epsilon}^{\delta_{0}}+\left(\frac{\epsilon}{\widetilde{\epsilon}}\right)^{2}\right),
\end{aligned}
$$

and choosing $\tilde{\epsilon}=\epsilon^{\alpha}$ with $\alpha=2 /\left(2+\delta_{0}\right)$, we get (6.17) with $\delta_{1}=2 \delta_{0} /\left(2+\delta_{0}\right)$.

7. Minimality to infinite order of critical points. Let $\Lambda_{0} \subset \mathbf{C}^{2 n}$ be an IR manifold and $p(x, \xi)$ a holomorphic function as in section 4 . We assume that $\Lambda_{0}$ is a critical point for the functional

$$
I(\Lambda, p)=\frac{1}{2} \int_{\Lambda} \log (p \bar{p}) \mu(d \rho)
$$

in the sense that

$$
\left\langle " d_{\Lambda} I\left(\Lambda_{0}, p\right) ", \delta \Lambda\right\rangle=\int_{\Lambda_{0}}\left\langle\left[H_{\arg p}\right]_{\Lambda_{0}}, d f\right\rangle \mu(d \rho)=0,
$$

for $f \in C_{b}^{\infty}(\Lambda ; \mathbf{R})$ corresponding to the infinitesimal variation $\Lambda_{0}+\delta \Lambda$. Equivalently,

$$
\operatorname{div}\left[H_{\arg p}\right]_{\Lambda_{0}}=0,
$$


where we recall that $\operatorname{div}\left[H_{\arg p}\right]_{\Lambda_{0}}$ is a distribution of order $\leq 1$ on $\Lambda_{0}$ with support in $p^{-1}(0) \cap \Lambda_{0}$. We assume that $\Lambda_{0}$ is a regular point in the sense that there exists $\delta_{1}>0$ such that

$$
[d \arg p]_{\Lambda_{0}}-\frac{p \bar{p}}{\epsilon^{2}+p \bar{p}}[d \arg p]_{\Lambda_{0}}=\mathcal{O}\left(\epsilon^{\delta_{1}}\right) \text { in } L^{1},
$$

and recall that this implies that

$$
\left\langle d_{\Lambda} I_{\epsilon}\left(\Lambda_{0}, p\right), \delta \Lambda\right\rangle-\left\langle " d_{\Lambda} I\left(\Lambda_{0}, p\right) ", \delta \Lambda\right\rangle=\mathcal{O}\left(\epsilon^{\delta_{1}}\right),
$$

when the form $d f$, corresponding to $\delta \Lambda$ is bounded. We also recall that (7.4) is a consequence of the property (B) of the preceding section.

Theorem 7.1. Assume (7.3), (7.4) and let

$$
\operatorname{neigh}\left(0, \mathbf{R}^{k}\right) \ni t \mapsto \Lambda_{t}
$$

be a smooth family of IR manifolds as in section 4 , with $\Lambda_{t=0}=\Lambda_{0}$. Then

$$
I\left(\Lambda_{t}, p\right)-I\left(\Lambda_{0}, p\right) \geq-C_{N}|t|^{N},
$$

for every $N \in \mathbf{N}$

Proof. Since our estimates will be uniform w.r.t. additional parameters, we may assume that $k=1$. The next result says that up to an error $\mathcal{O}\left(t^{\infty}\right)$ it is possible to obtain $\Lambda_{t}$ as the result of an autonomous almost holomorphic flow acting on $\Lambda_{0}$, with a real generator (on $\Lambda_{0}$ ).

Proposition 7.2. There exists $f_{t}(\rho)=f(t, \rho) \in C_{b}^{\infty}\left(\right.$ neigh $\left.(0, \mathbf{R}) \times \mathbf{C}^{2 n}\right)$, realvalued on $\Lambda_{0}$ and almost holomorphic in $\rho$ on $\Lambda_{0}$, such that

$$
\operatorname{dist}\left(\Lambda_{t}, \exp \left(t \widehat{t i H}_{f_{t}}\right)\left(\Lambda_{0}\right)\right)=\mathcal{O}\left(t^{\infty}\right) .
$$

Moreover $d f_{t}$ is unique on $\Lambda_{0} \bmod \mathcal{O}\left(t^{\infty}\right)$.

Proof. We first consider the situation locally near a point $\left(x_{0}, \xi_{0}\right) \in \Lambda_{0}$. After a complex canonical transformation, we may assume that $\Lambda_{t}$ is given by

$$
\xi=\frac{2}{i} \frac{\partial H_{t}}{\partial x}(x),
$$

where $H_{t}(x)$ is real and smooth in $(t, x)$ and strictly plurisubharmonic in $x$. Let $f \in C^{\infty}\left(\Lambda_{0} ; \mathbf{R}\right)$ and extend $f$ to a smooth function on $\mathbf{C}^{2 n}$, almost holomorphic at $\Lambda_{0}$. Then up to $\mathcal{O}\left(t^{\infty}\right), \widetilde{\Lambda}_{t}=\exp \left(t \widehat{i H_{f}}\right)\left(\Lambda_{0}\right)$ is given by

$$
\xi=\frac{2}{i} \frac{\partial G_{t}}{\partial x}(x),
$$

where $G_{t}(x)=G(t, x)$ solves the Hamilton-Jacobi problem

$$
\partial_{t} G(t, x)-\operatorname{Re} f\left(x, \frac{2}{i} \partial_{x} G(t, x)\right)=0, G(0, x)=H_{0}(x) .
$$


(Here we use that up to infinite order at $\Lambda_{0}$, we have $\widehat{i H_{f}}=H_{\operatorname{Re} f}^{\operatorname{Im} \sigma}=H_{-\operatorname{Re} f}^{-\operatorname{Im} \sigma}$.) Moreover, it follows that

$$
\operatorname{Re} f\left(x, \frac{2}{i} \partial_{x} G(t, x)\right)=f\left(x, \frac{2}{i} \partial_{x} G(t, x)\right)+\mathcal{O}\left(t^{\infty}\right),
$$

so (7.10) gives

$$
\partial_{t} G(t, x)-f\left(x, \frac{2}{i} \partial_{x} G(t, x)\right)=\mathcal{O}\left(t^{\infty}\right), G(0, x)=H_{0}(x) .
$$

Notice that

$$
G(t, x)=H_{0}(x)+t f\left(x, \frac{2}{i} \partial_{x} H_{0}(x)\right)+\mathcal{O}\left(t^{2}\right) .
$$

If we let $f=f_{s}$ depend smoothly on a real parameter $s$, then differentiating (7.12), we get $\partial_{s} G_{\mid t=0}=0$,

$$
\partial_{t} \partial_{s} G-\frac{2}{i} \partial_{\xi} f_{s}\left(x, \frac{2}{i} \partial_{x} G(t, x)\right) \cdot \partial_{x} \partial_{s} G(t, x)=\left(\partial_{s} f_{s}\right)\left(x, \frac{2}{i} \partial_{x} G(t, x)\right)+\mathcal{O}\left(t^{\infty}\right),
$$

and it follows that

$$
\partial_{s} G=t \partial_{s} f_{s}\left(x, \frac{2}{i} \partial_{x} H_{0}\right)+\mathcal{O}\left(t^{2}\right) .
$$

So if we replace $f(x, \xi)$ by $f(x, \xi)+\epsilon k(x, \xi)$, then $G(t, x)$ is replaced by

$$
G(t, x)+t \epsilon k\left(x, \frac{2}{i} \partial_{x} H_{0}\right)+\mathcal{O}\left(t^{2} \epsilon\right)+\mathcal{O}\left(t \epsilon^{2}\right) .
$$

It is now clear how to get the local existence of a suitable $f_{s}$ in (7.7) by successive approximations. We start by choosing (cf. $(7.13)) f^{(2)}(x, \xi)$ so that

$$
G(t, x)=H(t, x)+\mathcal{O}\left(t^{2}\right) .
$$

Then (with $\epsilon=s$ ), we try a corrected

$$
f_{s}^{(3)}=f^{(2)}(x, \xi)+s k(x, \xi) .
$$

Then (with $s=t), G(t, x)$ is replaced by

$$
G(t, x)+t^{2} k\left(x, \frac{2}{i} \partial_{x} H_{0}(x)\right)+\mathcal{O}\left(t^{3}\right),
$$

so there is a unique choice of $k$ so that for the new $G(t, x)$, we have

$$
G(t, x)=H(t, x)+\mathcal{O}\left(t^{3}\right) .
$$

Now we take $\epsilon=s^{2}$ and try a corrected

$$
f_{s}^{(4)}(x, \xi)=f^{(3)}(x, \xi)+s^{2} k(x, \xi),
$$

with a new $k$. Then $G(t, x)$ in (7.19) is replaced by

$$
G(t, x)+t^{3} k\left(x, \frac{2}{i} \partial_{x} H_{0}(x)\right)+\mathcal{O}\left(t^{4}\right),
$$


and there is a unique $k$ so that the new $G$ satisfies

$$
G(t, x)=H(t, x)+\mathcal{O}\left(t^{4}\right) .
$$

Continuing this way we get the local existence of $f_{s}$.

We next look at the local uniqueness. Let $f_{s}(x, \xi), g_{s}(x, \xi)$ be two functions which are almost holomorphic in $(x, \xi)$ at $\Lambda_{0}$, smooth in $(s, x, \xi)$ and real-valued for $(x, \xi) \in \Lambda_{0}$. Let $k \in \mathbf{N}$ be such that $f_{s}(x, \xi)-g_{s}(x, \xi)=s^{k} h(x, \xi)+\mathcal{O}\left(s^{k+1}\right)$ for some smooth function $h$. Then the two varieties $\exp \left(t i \widehat{H}_{f_{t}}\right)\left(\Lambda_{0}\right), \exp \left(t \widehat{H_{g_{t}}}\right)\left(\Lambda_{0}\right)$ are given $\left(\bmod . \mathcal{O}\left(t^{\infty}\right)\right)$ by $\xi=\frac{2}{i} \partial_{x} H(t, x)$ and $\xi=\frac{2}{i} \partial_{x} G(t, x)$, with

$$
H_{t}(x)-G_{t}(x)=t^{k+1} h\left(x, \frac{2}{i} \partial_{x} H_{0}(x)\right)+\mathcal{O}\left(t^{k+2}\right),
$$

and if $H=G$, it follows that $h\left(x, \frac{2}{i} \partial_{x} H_{0}(x)\right)=0$, and hence (since $\Lambda_{0}$ is maximally totally real), that $h=\mathcal{O}\left(\operatorname{dist}\left(\cdot, \Lambda_{0}\right)^{\infty}\right)$. (That we have uniqueness only up to a $t$ dependent constant is due to the fact that $H_{t}(x)$ is unique only up to such a constant.)

As already used in the proof above, we may replace $\exp \left(t \widehat{H_{f_{t}}}\left(\Lambda_{0}\right)\right)$ in the last proposition by $\widetilde{\Lambda}_{t, t}$, where $\widetilde{\Lambda}_{t, s}=\exp s\left(H_{\operatorname{Re} f_{t}}^{\operatorname{Im} \sigma}\right)\left(\Lambda_{0}\right)$. According to (5.18), we have

$$
\begin{aligned}
\partial_{s}^{2} \int_{\widetilde{\Lambda}_{t, s}} F(p) \mu(d \rho)=4 \int_{\widetilde{\Lambda}_{t, s}} & \frac{\partial F}{\partial \bar{p} \partial p}(p) H_{\widetilde{f}_{t, s}}(p) H_{\widetilde{f}_{t, s}}(\bar{p}) \mu(d \rho) \\
& +i \int_{\widetilde{\Lambda}_{t, s}}\left[H_{p}\left(\frac{\partial F}{\partial p}\right)-H_{\bar{p}}\left(\frac{\partial F}{\partial \bar{p}}\right)\right] \partial_{s} \widetilde{f}_{t, s} \mu(d \rho),
\end{aligned}
$$

where $\widetilde{f}_{t, s}$ denotes an almost holomorphic extension of $\operatorname{Re} f_{t}$ from $\widetilde{\Lambda}_{t, s}$ and all the Hamilton fields are in the sense of the symplectic manifold $\widetilde{\Lambda}_{t, s}$. Now $f_{t}$ is almost holomorphic on $\Lambda_{0}$ and $f_{t}=\operatorname{Re} f_{t}+\mathcal{O}\left(s^{\infty}\right)$ on $\widetilde{\Lambda}_{t, s}$. Consequently, $\nabla_{s, \rho}\left(\tilde{f}_{t, s}(\rho)-\right.$ $\left.f_{t}(\rho)\right)=\mathcal{O}\left(s^{\infty}\right)$ on $\widetilde{\Lambda}_{t, s}$ and in particular

$$
\partial_{s} \widetilde{f}_{t, s}=\mathcal{O}\left(s^{\infty}\right) \text { on } \widetilde{\Lambda}_{t, s}
$$

We shall use this in $(7.22)$ with $F(\rho)=\frac{1}{2} \log \left(\frac{\epsilon^{2}+p \bar{p}}{\epsilon^{2}+1}\right)$. Since $\frac{\partial^{2} F}{\partial \bar{p} \partial p} \geq 0$ (as we shall review in the beginning of section 8$)$, the first term to the right will be $\geq 0$. The second derivatives of $F$ are $\mathcal{O}\left(\epsilon^{-2}\right)$ on some compact subset of $\widetilde{\Lambda}_{t, s}$ and uniformly bounded outside, so the last term in $(7.22)$ is $\mathcal{O}\left(s^{M} / \epsilon^{2}\right)$ for every $M \in \mathbf{N}$. It follows that

$$
I_{\epsilon}\left(\widetilde{\Lambda}_{t, t}, p\right)-I_{\epsilon}\left(\Lambda_{0}, p\right) \geq t\left(\partial_{s}\right)_{s=0} I_{\epsilon}\left(\widetilde{\Lambda}_{t, s}, p\right)-\mathcal{O}_{M}(1) \frac{t^{M}}{\epsilon^{2}}
$$

Here

$$
\begin{aligned}
& \left(\partial_{s}\right)_{s=0} I_{\epsilon}\left(\widetilde{\Lambda}_{t, s}, p\right)=\left(\partial_{s}\right)_{s=0}\left(I_{\epsilon}\left(\widetilde{\Lambda}_{t, s}, p\right)-I_{0}\left(\widetilde{\Lambda}_{t, s}, p\right)\right) \\
& =-\int_{\Lambda_{0}}\left\langle[d \arg p]_{\Lambda_{0}}-\frac{p \bar{p}}{\epsilon^{2}+p \bar{p}}[d \arg p]_{\Lambda_{0}}, H_{\widetilde{f}_{t, 0}}\right\rangle \mu(d \rho)=\mathcal{O}\left(\epsilon^{\delta_{1}}\right)
\end{aligned}
$$

according to $(7.3),(7.4)$, and using this in (7.24) together with the fact that $I_{\epsilon}\left(\widetilde{\Lambda}_{t, t}, p\right)-$ $I_{\epsilon}\left(\Lambda_{t}, p\right)=\mathcal{O}\left(t^{\infty}\right)$ (since $\operatorname{dist}\left(\widetilde{\Lambda}_{t, t}, \Lambda_{t}\right)=\mathcal{O}\left(t^{\infty}\right)$ and $\Lambda \mapsto I_{\epsilon}(\Lambda, p)$ is uniformly Lipschitz), we get

$$
I_{\epsilon}\left(\Lambda_{t}, p\right)-I_{\epsilon}\left(\Lambda_{0}, p\right) \geq-\mathcal{O}\left(\epsilon^{\delta_{1}}\right)|t|-\mathcal{O}_{M}(1) \frac{t^{M}}{\epsilon^{2}}
$$


Finally we use that $I_{\epsilon}(\Lambda, p)-I(\Lambda, p)=\mathcal{O}\left(\epsilon^{1 / m}\right)$ (see Lemma 4.2), to get

$$
I\left(\Lambda_{t}, p\right)-I\left(\Lambda_{0}, p\right) \geq-\mathcal{O}_{M}\left(\epsilon^{1 / m}+\epsilon^{\delta_{1}}+\frac{t^{M}}{\epsilon^{2}}\right) .
$$

Choosing first $\epsilon=t^{K}$ with $K$ very large, and then $M$ sufficiently large, we obtain (7.6) with $N$ as large as we like, and the proof of Theorem 7.1 is complete.

Assume that $p(\rho)=p(z, \rho)$ depends holomorphically on $z \in \Omega \subset \mathbf{C}$. It is clear that $I(\Lambda, p(z))$ is subharmonic in $z$ for every fixed $\Lambda$. We end this section by giving a completely formal argument which indicates that if we have a minimizer $\Lambda=\Lambda(z)$ to $\Lambda \mapsto I(\Lambda, p(z))$, then $I(\Lambda(z), p(z))$ will also be subharmonic. The argument is formal but it should be possible to turn it into a proof whenever we have a sufficiently good control over the variational problem:

For $\Lambda$ close to $\Lambda(0)$, we have

$$
\Lambda=\Lambda_{f}=\exp \left(\widehat{i H_{f}}\right)(\Lambda(0))
$$

to infinite order at $\Lambda(0)$, where $f \in C_{b}^{\infty}(\Lambda(0) ; \mathbf{R})$ is small and we also let $f$ denote an almost holomorphic extension. If we allow $f$ to be complex valued, we still get an IR-manifold, close to $\Lambda(0)$, and we see that $I\left(\Lambda_{f}, p(z)\right)=I(f, z)$ is plurisubharmonic in $(z, f)$. At $z=0, f=0$, the Hessian (that we assume exists) of $I(f, z)$ is a quadratic form in $(\operatorname{Re} f, z)$ and the plurisubharmonicity means that

$$
\left(\begin{array}{cc}
\frac{1}{4} I_{f f}^{\prime \prime} & \frac{1}{2} I_{f z}^{\prime \prime} \\
\frac{1}{2} I_{z f}^{\prime \prime} & I_{\bar{z} z}^{\prime \prime}
\end{array}\right) \geq 0
$$

Here the subscript $f$ indicates ordinary (real) derivatives with respect to $f$, while $z$ and $\bar{z}$ indicate holomorphic and anti-holomorphic derivatives (as in the Levi form). In other terms:

$$
0 \leq \frac{1}{4}\left(I_{f f}^{\prime \prime} \phi \mid \phi\right)+\frac{1}{2}\left(I_{f z}^{\prime \prime} \zeta \mid \phi\right)+\frac{1}{2}\left(I_{\bar{z} f}^{\prime \prime} \phi \mid \zeta\right)+\left(I_{\bar{z} z}^{\prime \prime} \zeta \mid \zeta\right)
$$

for all tangent vectors $(\phi, \zeta)$, where we use standard sesquilinear scalar products.

Assume (for instance after an arbitrarily small convexification in $f$ ) that $I_{f f}^{\prime \prime}>0$. For $z$ close to 0 , let $f(z)$ be the real function with $\Lambda(z)=\Lambda_{f(z)}$, so that $I_{f}^{\prime}(f(z), z)=0$. Differentiating, we get $0=I_{f f}^{\prime \prime} f_{z}^{\prime}+I_{f z}^{\prime \prime}, f_{z}^{\prime}=-\left(I_{f f}^{\prime \prime}\right)^{-1} I_{f z}^{\prime \prime}$. On the other hand, $(I(f(z), z))_{\bar{z}}^{\prime}=I_{\bar{z}}^{\prime}(f(z), z)$, so that

$$
(I(f(z), z))_{z z}^{\prime \prime}=I_{\bar{z} z}^{\prime \prime}(f(z), z)+I_{z}^{\prime \prime}(f(z), z) f_{z}^{\prime}(z)=I_{z z}^{\prime \prime}-I_{z}^{\prime \prime}\left(I_{f f}^{\prime \prime}\right)^{-1} I_{f z}^{\prime \prime} .
$$

Choosing $\phi=-2\left(I_{f f}^{\prime \prime}\right)^{-1} I_{f z}^{\prime \prime} \zeta$ in $(7.28)$, we see that this quantity is $\geq 0$.

8. The codimension 2 case. We start by recalling a very classical formula and its proof. Let $\epsilon>0$. Then for $p \in \mathbf{C}$ :

$$
\partial_{p} \partial_{\bar{p}} \log \left(\epsilon^{2}+p \bar{p}\right)=\partial_{p} \frac{p}{\epsilon^{2}+p \bar{p}}=\frac{1}{\epsilon^{2}+p \bar{p}}-\frac{p \bar{p}}{\left(\epsilon^{2}+p \bar{p}\right)^{2}}=\frac{\epsilon^{2}}{\left(\epsilon^{2}+p \bar{p}\right)^{2}} .
$$

This is a non-negative function which tends to 0 for $p \neq 0$. If $L(d p)$ denotes the Lebesgue measure on $\mathbf{C}$, we have

$$
\begin{aligned}
& \int_{\mathbf{C}} \frac{\epsilon^{2}}{\left(\epsilon^{2}+p \bar{p}\right)^{2}} L(d p)=2 \pi \int_{0}^{\infty} \frac{\epsilon^{2}}{\left(\epsilon^{2}+r^{2}\right)^{2}} r d r= \\
& \pi \int_{0}^{\infty} \frac{\epsilon^{2}}{\left(\epsilon^{2}+t\right)^{2}} d t=\pi\left[-\frac{\epsilon^{2}}{\left(\epsilon^{2}+t\right)}\right]_{t=0}^{\infty}=\pi .
\end{aligned}
$$


We conclude that $\log \left(\epsilon^{2}+p \bar{p}\right)$ is subharmonic for $0 \leq \epsilon \leq 1$, and that

$$
\partial_{p} \partial_{\bar{p}} \log (p \bar{p})=\pi \delta .
$$

Let $\Lambda \subset \mathbf{C}^{2 n}$ be an IR manifold, and let $p$ be a holomorphic function as in section 4. Recall that

$$
I_{\epsilon}(\Lambda, p)=\frac{1}{2} \int_{\Lambda} \log \left(\frac{\epsilon^{2}+p \bar{p}}{\epsilon^{2}+1}\right) \mu(d \rho), I_{0}=I .
$$

If $p=p(\rho, z)$ depends holomorphically on a complex parameter $z$, we get for $\epsilon>0$ :

$$
\begin{aligned}
& \partial_{z} \partial_{\bar{z}} I_{\epsilon}(\Lambda, p)=\frac{1}{2} \int_{\Lambda} \partial_{p} \partial_{\bar{p}}\left(\log \left(\epsilon^{2}+p \bar{p}\right)\right) \partial_{z} p \overline{\partial_{z} p} \mu(d \rho) \\
& =\frac{1}{2} \int_{\Lambda} \frac{\epsilon^{2}}{\left(\epsilon^{2}+p \bar{p}\right)^{2}} \partial_{z} p \overline{\partial_{z} p} \mu(d \rho),
\end{aligned}
$$

so $I_{\epsilon}(\Lambda, p)$ is a subharmonic function of $z$. This also holds for the limiting case $\epsilon=0$.

We shall investigate the effect of small variations of $\Lambda$. Let $\Lambda=\Lambda_{0}$, where ]$-\delta, \delta\left[\ni t \mapsto \Lambda_{t}\right.$ is a smooth deformation as in section 4 . Let $\widehat{H_{i f_{t}}}$ be the corresponding deformation field, $f_{t} \in C_{b}^{\infty}\left(\Lambda_{t} ; \mathbf{R}\right)$ and $f_{t}$ also denotes a corresponding almost holomorphic extension in $\rho$. Let $\kappa_{t}: \Lambda_{0} \rightarrow \Lambda_{t}$ be the corresponding flow, so that $\kappa_{t}$ is symplectic. Repeating earlier calculations,

$$
I_{\epsilon}\left(\Lambda_{t}, p\right)=I_{\epsilon}\left(\Lambda_{0}, p_{t}\right), \text { where } p_{t}=p \circ \kappa_{t} .
$$

Since

$$
\partial_{t} p_{t}=i H_{f_{t}}(p) \circ \kappa_{t}
$$

we get

$$
\begin{aligned}
& \partial_{t} I_{\epsilon}\left(\Lambda_{t}, p\right)= \\
& \frac{1}{2} \int_{\Lambda_{0}} \partial_{p}\left(\log \left(\epsilon^{2}+p_{t} \bar{p}_{t}\right)\right) i H_{f_{t}}(p) \circ \kappa_{t} \mu(d \rho)+\frac{1}{2} \int_{\Lambda_{0}} \partial_{\bar{p}}\left(\log \left(\epsilon^{2}+p_{t} \bar{p}_{t}\right)\right) \overline{i H_{f_{t}} p \circ \kappa_{t} \mu(d \rho)} \\
& =\operatorname{Re} \int_{\Lambda_{0}} \partial_{p}\left(\log \left(\epsilon^{2}+p_{t} \bar{p}_{t}\right)\right) i H_{f_{t}}(p) \circ \kappa_{t} \mu(d \rho) .
\end{aligned}
$$

This can also be written

$$
\partial_{t} I_{\epsilon}\left(\Lambda_{t}, p\right)=\operatorname{Re} \int_{\Lambda_{t}} \partial_{p}\left(\log \left(\epsilon^{2}+p \bar{p}\right)\right) i H_{f_{t}}(p) \mu(d \rho) .
$$

Take $t=0$ for simplicity, and use that $H_{f_{t}}(p)=-H_{p}\left(f_{t}\right)$, to get

$$
\left(\partial_{t}\right)_{t=0} I_{\epsilon}\left(\Lambda_{t}, p\right)=-\operatorname{Re} i \int_{\Lambda} \partial_{p}\left(\log \left(\epsilon^{2}+p \bar{p}\right)\right) H_{p}\left(f_{0}\right) \mu(d \rho),
$$

where $H_{p}$ is viewed as a differential operator on $\Lambda$, namely the Hamilton field of the restriction of $p$ to $\Lambda$ with respect to the restriction of $\sigma$ to $\Lambda$. In order not to make the notations too heavy, we shall often not distinguish between functions on $\mathbf{C}^{2 n}$ and their 
restrictions to $\Lambda$. Since the transpose of $H_{p}$ with respect to the symplectic volume form $\mu(d \rho)$ is equal to $-H_{p}$, we get

$$
\begin{aligned}
& \left(\partial_{t}\right)_{t=0} I_{\epsilon}\left(\Lambda_{t}, p\right)=\operatorname{Re} i \int_{\Lambda} f_{0} H_{p}\left(\partial_{p}\left(\log \left(\epsilon^{2}+p \bar{p}\right)\right)\right) \mu(d \rho) \\
& =\operatorname{Re} i \int_{\Lambda} f_{0}\left(\partial_{\bar{p}} \partial_{p}\left(\log \left(\epsilon^{2}+p \bar{p}\right)\right)\right)\{p, \bar{p}\} \mu(d \rho)=2 \int_{\Lambda} f_{0} \frac{\epsilon^{2}}{\left(\epsilon^{2}+p \bar{p}\right)^{2}} \frac{i}{2}\{p, \bar{p}\} \mu(d \rho) .
\end{aligned}
$$

Here $\{p, \bar{p}\}=\left\{p_{\Lambda}, \overline{p_{\Lambda}}\right\}$ denotes the Poisson bracket for the symplectic manifold $\Lambda$.

Throughout the remainder of this section, we shall assume with $p=p_{\Lambda}$ :

$$
d p, d \bar{p} \text { are independent at all points of } \Lambda \cap p^{-1}(0) .
$$

This implies that $\Sigma:=\Lambda \cap p^{-1}(0)$ is a smooth (possibly empty) submanifold of codimension 2 in $\Lambda$. Since $\Lambda$ is symplectic, it is orientable: We say that the volume form $\sigma^{n} / n$ ! is positive on $\Lambda$ (and hence identified with the volume density $\mu(d \rho)$ ). We then have a natural induced orientation on $\Sigma$ depending also on $p$ : We say that a $2 n-2$ form $\alpha$ on $\Sigma$ is positive at some point in $\Sigma$ if $\alpha \wedge \frac{i}{2} d p \wedge d \bar{p}$ is a positive multiple of $\sigma^{n} / n$ ! at that point. (Here we notice that $\frac{i}{2} d p \wedge d \bar{p}=d \operatorname{Re} p \wedge d \operatorname{Im} p$.) We define the Liouville measure on $\Sigma$ to be the density alias positive $2 n-2$ form $\lambda_{p, 0}$ on $\Sigma$, such that

$$
\lambda_{p, 0} \wedge \frac{i}{2} d p \wedge d \bar{p}=\frac{\sigma^{n}}{n !} .
$$

In the next lemma we continue to write $\{p, \bar{p}\}=\left\{p_{\Lambda}, \overline{p_{\Lambda}}\right\}$.

Lemma 8.1. For $n \geq 2$, we have

$$
\frac{i}{2}\{p, \bar{p}\} \lambda_{p, 0}=\left(\frac{\sigma^{n-1}}{(n-1) !}\right)_{\Sigma} .
$$

In particular, it follows that $\int_{\Gamma} \frac{i}{2}\{p, \bar{p}\} \lambda_{p, 0}(d \rho)=0$, for every connected component $\Gamma$ of $\Sigma$.

Proof. We know that $\Sigma$ is symplectic at a point $\rho \in \Sigma$ (i.e. $\sigma_{\left.\right|_{\Sigma}}$ is non-degenrate at $\rho$ ) iff $\{p, \bar{p}\} \neq 0$, and we know that $\left.\sigma^{n-1}\right|_{\Sigma}$ is non-vanishing precisely at the points where $\Sigma$ is symplectic. Consequently both members of (8.13) vanish at the points where $\Sigma$ is not symplectic. On the other hand, near a point where $\Sigma$ is symplectic, we can choose symplectic coordinates $x_{1}, . ., x_{n}, \xi_{1}, . ., \xi_{n}$ on $\Lambda$, so that $\Sigma$ is given by $x_{n}=$ $\xi_{n}=0$. Since $\lambda_{p, 0}$ is a form of maximal degree in $\left(x^{\prime}, \xi^{\prime}\right)=\left(x_{1}, . ., x_{n-1}, \xi_{1}, . ., \xi_{n-1}\right)$, we get from (8.12):

$$
\begin{aligned}
\frac{\sigma^{n}}{n !}=\lambda_{p, 0} \wedge \frac{i}{2} d p \wedge d \bar{p} & =\lambda_{p, 0} \wedge \frac{i}{2}\left(\frac{\partial p}{\partial \xi_{n}} d \xi_{n}+\frac{\partial p}{\partial x_{n}} d x_{n}\right) \wedge\left(\frac{\partial \bar{p}}{\partial \xi_{n}} d \xi_{n}+\frac{\partial \bar{p}}{\partial x_{n}} d x_{n}\right) \\
& =\frac{i}{2}\{p, \bar{p}\} \lambda_{p, 0} \wedge d \xi_{n} \wedge d x_{n} .
\end{aligned}
$$

Now use that

$$
\frac{\sigma^{n}}{n !}=d \xi_{1} \wedge d x_{1} \wedge d \xi_{2} \wedge d x_{2} \wedge . . \wedge d \xi_{n} \wedge d x_{n}
$$

to conclude that

$$
\frac{i}{2}\{p, \bar{p}\} \lambda_{p, 0}=d \xi_{1} \wedge d x_{1} \wedge . . \wedge d \xi_{n-1} \wedge d x_{n-1}=\left(\frac{\sigma^{n-1}}{(n-1) !}\right)_{\Sigma} .
$$


Next we consider the limits of (8.5), (8.11), when $\epsilon \rightarrow 0$. It is easy to see that the contribution from the region where $|p| \geq \delta$ is 0 , for every fixed $\delta>0$. On the other hand, in the region $|p|<\delta$, we introduce $\Sigma_{w}:=p^{-1}(w)$, for $w \in D(0, \delta):=\{\omega \in$ $\mathbf{C} ;|\omega|<\delta\}$, and the corresponding Liouville form $\lambda_{p, w}$. Then

$$
\lambda_{p, w} \wedge \frac{i}{2} d p \wedge d \bar{p}=\frac{1}{n !} \sigma^{n},
$$

at the points of $\Sigma_{w}$, and in view of (8.5):

$$
\begin{aligned}
& \frac{1}{2} \int_{\Lambda \cap p^{-1}(D(0, \delta))} \frac{\epsilon^{2}}{\left(\epsilon^{2}+p \bar{p}\right)^{2}} \partial_{z} p \overline{\partial_{z} p} \mu(d \rho)= \\
& \frac{1}{2} \int_{D(0, \delta)} \frac{\epsilon^{2}}{\left(\epsilon^{2}+w \bar{w}\right)^{2}}\left(\int_{\Sigma_{w}} \partial_{z} p \overline{\partial_{z} p} \lambda_{p, w}(d \rho)\right) \wedge d \operatorname{Re} w \wedge d \operatorname{Im} w .
\end{aligned}
$$

As we saw in the beginning of this section,

$$
\frac{\epsilon^{2}}{\left(\epsilon^{2}+w \bar{w}\right)^{2}} \rightarrow \pi \delta_{w=0}
$$

so the expression $\left(^{*}\right)$ converges to

$$
\frac{\pi}{2} \int_{\Sigma} \partial_{z} p \overline{\partial_{z} p} \lambda_{p, 0}(d \rho)
$$

We conclude that

$$
\partial_{z} \partial_{\bar{z}} I(\Lambda, p)=\frac{\pi}{2} \int_{\Lambda \cap p^{-1}(0)} \partial_{z} p \overline{\partial_{z} p} \lambda_{p, 0}(d \rho),
$$

first in the sense of distributions, then in the classical sense, since the RHS in this last equation is smooth.

The discussion also applies to (8.11), and shows that

$$
\left(\partial_{t}\right)_{\mid t=0} I\left(\Lambda_{t}, p\right)=2 \pi \int_{\Lambda \cap p^{-1}(0)} f_{0} \frac{i}{2}\{p, \bar{p}\} \lambda_{p, 0}(d \rho)=2 \pi \int_{\Lambda \cap p^{-1}(0)} f_{0} \frac{1}{(n-1) !} \sigma^{n-1} .
$$

We conclude that $\Lambda=\Lambda_{0}$ is a critical point for the functional $\Lambda \mapsto I(\Lambda, p)$ iff for $p=p_{\left.\right|_{\Lambda}}$, we have on $\Lambda$ :

$$
p(\rho)=0 \Rightarrow\{p, \bar{p}\}=0 .
$$

Combining (8.1) and the earlier discussion with (5.18) and the subsequent remark, we get

$$
\partial_{t}^{2} I\left(\Lambda_{t}, p\right)=2 \pi \int_{\Lambda_{t} \cap p^{-1}(0)} H_{p} f_{t} \overline{H_{p} f_{t}} \lambda_{p, 0}(d \rho)+2 \pi \int_{\Lambda_{t} \cap p^{-1}(0)}\left(\partial_{t} f_{t}\right) \frac{i}{2}\{p, \bar{p}\} \lambda_{p, 0}(d \rho) .
$$

If we extend the definition of $I\left(\Lambda_{t}, p\right)$ to complex $t$ by almost holomorphic extension of the flow $\kappa_{t}$, a simpler and more direct computation shows that

$$
\partial_{\bar{t}} \partial_{t} I\left(\Lambda_{t}, p\right)=\frac{\pi}{2} \int_{\Lambda_{t} \cap p^{-1}(0)} H_{p} f_{t} \overline{H_{p} f_{t}} \lambda_{p, 0}(d \rho) \text {, for } t \text { real. }
$$


In this last identity, we let $\partial_{t}$ and $\partial_{\bar{t}}$ denote holomorphic and antiholomorphic derivatives.

Let $\Lambda$ be critical for $I(\cdot, p)$ in the sense that we have (8.17). We shall next see that if $n=2$ and if we make an infinitesimal change $p \mapsto p+\delta p$, then there is a corresponding infinitesimal change $\Lambda \mapsto \exp \left(i H_{\delta f}\right)(\Lambda)=\widetilde{\Lambda}$ of $\Lambda$, such that $\widetilde{\Lambda}$ is critical for $I(\cdot, p+\delta p)$ :

THEOREM 8.2. Let $n=2$, let $\Lambda$ be an IR-manifold and $p$ a holomorphic function as in section 4. Assume $(H)$ and (8.17). Let $q$ be holomorphic and $\mathcal{O}\left(\langle(x, \xi)\rangle^{m}\right), m<$ $-2 n$, in some tubular neighborhood of $\Lambda$ and put $p_{z}(\rho)=p(\rho)+z q(\rho), z \in$ neigh $(0, \mathbf{R})$. Then there exists $f \in C_{b}^{\infty}(\Lambda ; \mathbf{R})$ such that if $\Lambda_{z}=\exp \left(z \widehat{i H_{f}}\right)(\Lambda)$, is the corresponding IR-deformation, we have

$$
\left\{p_{z}, \bar{p}_{z}\right\}=\mathcal{O}\left(z^{2}\right) \text { on } p_{z}^{-1}(0) \cap \Lambda_{z}
$$

Here the Poisson bracket is the one given by the symplectic form on $\Lambda_{z}$.

In a forthcoming paper, we shall show, by using non-linear $\bar{\partial}$ equations, a stronger version of the above result, namely that the $\mathcal{O}\left(z^{2}\right)$ can be replaced by 0 , for small $z$. We think that the proof below has some independent interest and reveals some intereresting structures. It is based on the use of second order elliptic operators rather than $\bar{\partial}$ type operators.

In proving the theorem, it will be convenient to use the terminology of infinitesimal variations $\delta p=z q, z \rightarrow 0$, and $\delta f=z f, z \rightarrow 0$, so that our calculations will be modulo errors $\mathcal{O}\left(z^{2}\right)$.

From (8.17) and $(\mathrm{H})$, we see that

$$
\{p, \bar{p}\}=\bar{a} p-a \bar{p}
$$

for some smooth function $a$ which is uniquely determined on $\Sigma$ (and where the Poisson bracket is the one of the symplectic manifold $\Lambda$ ). For a general infinitesimal change $\delta p$, of $p$, we get

$$
\begin{aligned}
& \{p+\delta p, \overline{p+\delta p}\}=\bar{a} p-a \bar{p}+\{\delta p, \bar{p}\}+\{p, \delta \bar{p}\}= \\
& \bar{a}(p+\delta p)-a(\bar{p}+\delta \bar{p})+\left(H_{p}+a\right)(\delta \bar{p})-\left(H_{\bar{p}}+\bar{a}\right)(\delta p)
\end{aligned}
$$

so $($ on $\Lambda)$

$$
\{p+\delta p, \overline{p+\delta p}\}=\left(H_{p}+a\right)(\delta \bar{p})-\left(H_{\bar{p}}+\bar{a}\right)(\delta p) \text { on }(p+\delta p)^{-1}(0) .
$$

If we apply an infinitesimal change $\Lambda \mapsto \widetilde{\Lambda}=\exp i H_{\delta f}(\Lambda)$, we get

$$
(p+\delta p)_{\left.\right|_{\Lambda}} \simeq p+\delta p+i H_{\delta f}(p)_{\left.\right|_{\Lambda}}=\left(p+\delta p-i H_{p}(\delta f)\right)_{\left.\right|_{\Lambda}},
$$

so we get

$$
\begin{aligned}
& \{p+\delta p, \overline{p+\delta p}\}_{\mid \widetilde{\Lambda} \cap(p+\delta p)^{-1}(0)} \simeq \\
& \left\{p+\delta p-i H_{p}(\delta f), \overline{p+\delta p-i H_{p}(\delta f)}\right\}_{\mid \Lambda \cap\left(p+\delta p-i H_{p}(\delta f)\right)^{-1}(0)}= \\
& \left(H_{p}+a\right)\left(\delta \bar{p}+i H_{\bar{p}} \delta f\right)-\left(H_{\bar{p}}+\bar{a}\right)\left(\delta p-i H_{p} \delta f\right)
\end{aligned}
$$


where we used (8.21) with a new infinitesimal change in the last step. Since the last expression is already infinitely small, we can ignore the effect of the infinitesimal displacement of the zero set of $p$ and consider that it is given on $p^{-1}(0)$. To have it equal to 0 amounts to solving on $p^{-1}(0)$ the equation

$$
i\left(\left(H_{p}+a\right) H_{\bar{p}}+\left(H_{\bar{p}}+\bar{a}\right) H_{p}\right) \delta f=-\left(H_{p}+a\right) \delta \bar{p}+\left(H_{\bar{p}}+\bar{a}\right) \delta p
$$

or in other terms

$$
\operatorname{Re}\left(H_{p}+a\right) H_{\bar{p}} \delta f=-\operatorname{Im}\left(H_{p}+a\right) \delta \bar{p} \text { on } p^{-1}(0) \cap \Lambda .
$$

Here $\left(H_{p}+a\right) H_{\bar{p}}$ is an elliptic second order operator on the leaves of the bicharacteristic foliation of $\Sigma=p^{-1}(0) \cap \Lambda$, so when $n=2$, it is an elliptic operator on $\Sigma$. Let us notice that it is real on $\Sigma$ :

$$
2 i \operatorname{Im}\left(H_{p}+a\right) H_{\bar{p}}=\left(H_{p}+a\right) H_{\bar{p}}-\left(H_{\bar{p}}+\bar{a}\right) H_{p}=\left[H_{p}, H_{\bar{p}}\right]-\left(\bar{a} H_{p}-a H_{\bar{p}}\right)=0,
$$

where in the last step we used (8.19) and the fact that $\left[H_{p}, H_{\bar{p}}\right]=H_{\{p, \bar{p}\}}$. Since $\delta f$ is real the problem (8.23) reduces to

$$
-\left(H_{p}+a\right) H_{\bar{p}}(\delta f)=\operatorname{Im}\left(H_{p}+a\right) \delta \bar{p} \text { on } \Sigma .
$$

Lemma 8.3. For every smooth function $u$ on $\Sigma$ and every component $\Gamma$ of $\Sigma$, we have

$$
\int_{\Gamma}\left(H_{p}+a\right) u \lambda_{p, 0}(d \rho)=0 .
$$

In particular, if we replace $u$ by a product $u \bar{v}$, we see that

$$
\left(H_{p}+a\right)^{*}=-H_{\bar{p}},
$$

where the star indicates that we take the complex adjoint in $L^{2}\left(\Gamma, \lambda_{p, 0}(d \rho)\right)$.

Proof. We compute the Lie derivative $\mathcal{L}_{H_{p}}\left(\lambda_{p, 0}\right)$ : First we recall that $\mathcal{L}_{H_{p}} \sigma=0$ and hence $\mathcal{L}_{H_{p}} \sigma^{n}=0$. Secondly, at the points of $\Sigma$, we get

$$
\mathcal{L}_{H_{p}}(d p \wedge d \bar{p})=d p \wedge d\{p, \bar{p}\}=d p \wedge(\bar{a} d p-a d \bar{p})=-a d p \wedge d \bar{p} .
$$

Still at the points of $\Sigma$, we apply $\mathcal{L}_{H_{p}}$ to the relation

$$
\lambda_{p, 0} \wedge \frac{i}{2} d p \wedge d \bar{p}=\frac{1}{n !} \sigma^{n},
$$

and get

$0=\mathcal{L}_{H_{p}}\left(\lambda_{p, 0}\right) \wedge \frac{i}{2} d p \wedge d \bar{p}+\lambda_{p, 0} \wedge \frac{i}{2} \mathcal{L}_{H_{p}}(d p \wedge d \bar{p})=\mathcal{L}_{H_{p}}\left(\lambda_{p, 0}\right) \wedge \frac{i}{2} d p \wedge d \bar{p}-a \lambda_{p, 0} \wedge \frac{i}{2} d p \wedge d \bar{p}$.

It follows that

$$
\mathcal{L}_{H_{p}}\left(\lambda_{p, 0}\right)=a \lambda_{p, 0}
$$


To get (8.25), we write

$$
0=\int_{\Gamma} \mathcal{L}_{H_{p}}\left(u \lambda_{p, 0}(d \rho)\right)=\int_{\Gamma}\left(H_{p}(u) \lambda_{p, 0}+u \mathcal{L}_{H_{p}} \lambda_{p, 0}\right)=\int_{\Gamma}\left(H_{p}+a\right) u \lambda_{p, 0}(d \rho) .
$$

The operator in the LHS of (8.24) can be written in different forms:

$$
-\left(H_{p}+a\right) H_{\bar{p}}=\left(H_{\bar{p}}\right)^{*} H_{\bar{p}}=H_{p}^{*} H_{p}
$$

Here the first equality follows from (26) and second one from the fact that the operator is real. In particular, our operator is formally self-adjoint. If $u \in C^{\infty}(\Gamma)$, where $\Gamma$ is a component of $\Sigma$, and $\left(H_{p}+a\right) H_{\bar{p}} u=0$, we get

$$
\left\|H_{\bar{p}} u\right\|_{L^{2}\left(\Gamma, \lambda_{p, 0}\right)}^{2}=\left\|H_{p} u\right\|_{L^{2}\left(\Gamma, \lambda_{p, 0}\right)}^{2}=0,
$$

So $H_{\operatorname{Re} p} u=H_{\operatorname{Im} p} u=0$. This means that $u$ is constant on every bicharacteristic leaf and hence on the whole component $\Gamma$, since $n=2$. Let us now check that the RHS in (8.24) is orthogonal to $C^{\infty}(\Gamma) \cap \operatorname{Ker}\left(-\left(H_{p}+a\right) H_{\bar{p}}\right)$ also for general $n$ : The latter space is the complexification of its maximal subspace of real functions, and for such a real function $u$, we get

$$
\left(\operatorname{Im}\left(H_{p}+a\right) \delta \bar{p} \mid u\right)=\operatorname{Im}\left(\left(H_{p}+a\right) \delta \bar{p} \mid u\right)=-\operatorname{Im}\left(\delta \bar{p} \mid H_{\bar{p}} u\right)=0 .
$$

Returning to the case $n=2$, our operator is elliptic and essentially self-adjoint. The previous discussion implies that for every $\delta p \in C^{\infty}(\Gamma)$, the equation (8.24) has a solution $\delta f \in C^{\infty}(\Gamma)$, which is unique up to a constant. Theorem 8.2 follows.

Let $p_{z}$ be as in Theorem 8.2, now with $z \in \operatorname{neigh}(0, \mathbf{C})$ and assume that $p_{z}$ is holomorphic in $z$. We want to choose $z_{j} \mapsto f_{j}, j=1$, , so that with $z=z_{1}+i z_{2}, \Lambda_{z}$ is critical for $I\left(\cdot, p_{z}\right)$, where $\Lambda_{w}=\exp i\left(w_{1} H_{f_{1}}+w_{2} H_{f_{2}}\right)\left(\Lambda_{0}\right)$, and where $f_{j}$ are real and smooth on $\Lambda_{0}$ and the Hamilton flow is defined to infinite order at $\Lambda_{0} \times\{w=0\}$. We will only consider the infinitesimal solution of the problem, that we obtain from (8.24), so that for $z=w=0$ :

$$
\begin{aligned}
& -\left(H_{p}+a\right) H_{\bar{p}} f_{1}=\frac{1}{2 i}\left(H_{p}+a\right) \overline{\frac{\partial p}{\partial \operatorname{Re} z}}-\frac{1}{2 i}\left(H_{\bar{p}}+\bar{a}\right) \frac{\partial p}{\partial \operatorname{Re} z}, \\
& -\left(H_{p}+a\right) H_{\bar{p}} f_{2}=\frac{1}{2 i}\left(H_{p}+a\right) \overline{\frac{\partial p}{\partial \operatorname{Im} z}}-\frac{1}{2 i}\left(H_{\bar{p}}+\bar{a}\right) \frac{\partial p}{\partial \operatorname{Im} z} .
\end{aligned}
$$

Equivalently we have for $z=w=0$ :

$$
\begin{aligned}
& -\left(H_{p}+a\right) H_{\bar{p}} f_{w}=-\frac{1}{2 i}\left(H_{\bar{p}}+\bar{a}\right)\left(\frac{\partial p}{\partial z}\right) \\
& -\left(H_{p}+a\right) H_{\bar{p}} f_{\bar{w}}=\frac{1}{2 i}\left(H_{p}+a\right)\left(\frac{\overline{\partial p}}{\partial z}\right)
\end{aligned}
$$

where $f_{w}=\frac{1}{2}\left(f_{1}+\frac{1}{i} f_{2}\right)$. We then know that for the function $I\left(\Lambda_{w}, p_{z}\right)$,

$$
\nabla_{z}\left(\left(\nabla_{w} I\right)\left(\Lambda_{z}, p_{z}\right)\right)=0, \text { at } z=0
$$


or equivalently that

$$
\left(\left(\nabla_{z}+\nabla_{w}\right) \nabla_{w} I\right)\left(\Lambda_{w}, p_{z}\right)=0, \text { at } z=w=0
$$

which can be expanded in terms of holomorphic and antiholomorphic derivatives as:

$$
\begin{aligned}
& \left(\partial_{z} \partial_{w}+\partial_{w}^{2}\right) I=0,\left(\partial_{z} \partial_{\bar{w}}+\partial_{w} \partial_{\bar{w}}\right) I=0, \\
& \left(\partial_{\bar{z}} \partial_{w}+\partial_{\bar{w}} \partial_{w}\right) I=0,\left(\partial_{\bar{z}} \partial_{\bar{w}}+\partial_{\bar{w}}^{2}\right) I=0, \text { for } z=w=0 .
\end{aligned}
$$

From these relations we see that $\partial_{z} \partial_{\bar{w}} I=\partial_{\bar{z}} \partial_{w} I$ is real (at $z=w=0$ ) and that:

$$
\partial_{\bar{z}} \partial_{z}\left(I\left(\Lambda_{z}, p_{z}\right)\right)=\left(\partial_{\bar{z}}+\partial_{\bar{w}}\right)\left(\partial_{z}+\partial_{w}\right) I\left(\Lambda_{w}, p_{z}\right)=\partial_{\bar{z}} \partial_{z} I\left(\Lambda_{w}, p_{z}\right)-\partial_{\bar{w}} \partial_{w} I\left(\Lambda_{w}, p_{z}\right) .
$$

Since

$$
\frac{\partial}{\partial \bar{w}} \frac{\partial}{\partial w}=\frac{1}{4}\left(\left(\frac{\partial}{\partial w_{1}}\right)^{2}+\left(\frac{\partial}{\partial w_{2}}\right)^{2}\right)
$$

we have for $z=w=0$ :

$$
\begin{aligned}
\left(\frac{\partial}{\partial \bar{w}} \frac{\partial}{\partial w}\right) I\left(\Lambda_{w}, p_{z}\right) & =\frac{1}{4}\left(\left(\frac{\partial}{\partial w_{1}}\right)^{2} I_{1}\left(w_{1}\right)+\left(\frac{\partial}{\partial w_{2}}\right)^{2} I_{2}\left(w_{2}\right)\right), \\
I_{j}\left(w_{j}\right) & =\left\{\begin{array}{l}
I\left(\Lambda_{\left(w_{1}, 0\right)}, p_{0}\right), j=1 \\
I\left(\Lambda_{\left(0, w_{2}\right)}, p_{0}\right), j=2 .
\end{array}\right.
\end{aligned}
$$

In the definition of $I_{j}\left(w_{j}\right)$ we can let $w_{j}$ be complex and we notice that to infinite order at $w_{j}=0$, we have $I_{j}\left(w_{j}\right) \equiv I_{j}\left(\operatorname{Re} w_{j}\right)$. Hence for $w_{j}=0, z=0$ :

$$
\begin{aligned}
& \left(\frac{\partial}{\partial w_{1}}\right)^{2} I_{1}\left(w_{1}\right)=\left(\left(\frac{\partial}{\partial \operatorname{Re} w_{1}}\right)^{2}+\left(\frac{\partial}{\partial \operatorname{Im} w_{1}}\right)^{2}\right) I_{1}\left(w_{1}\right)= \\
& 4 \frac{\partial}{\partial \bar{w}_{1}} \frac{\partial}{\partial w_{1}} I_{1}\left(w_{1}\right)=4 \frac{\pi}{2} \int_{p^{-1}(0)} H_{p} f_{1} \overline{H_{p} f_{1}} \lambda_{p, 0}(d \rho),
\end{aligned}
$$

where we abused the notation since $\frac{\partial}{\partial w_{1}}, \frac{\partial}{\partial \bar{w}_{1}}$ in the third member denote holomorphic and antiholomorphic derivatives, and where we used (8.18) in the last step. The same calculation is valid for $j=2$, and we get for $z=w=0$ :

$\partial_{\bar{w}} \partial_{w} I=\frac{\pi}{2} \sum_{j=1}^{2} \int_{p^{-1}(0)} H_{p} f_{j} \overline{H_{p} f_{j}} \lambda_{p, 0}(d \rho)=-\frac{\pi}{2} \sum_{j=1}^{2} \int_{p^{-1}(0)}\left(\left(H_{\bar{p}}+\bar{a}\right) H_{p} f_{j}\right) \overline{f_{j}} \lambda_{p, 0}(d \rho)$.

Here we used again that the adjoint of $H_{p}$ for $\lambda_{p, 0}$ is $-\left(H_{\bar{p}}+\bar{a}\right)$. Now recall that $\left(H_{\bar{p}}+\bar{a}\right) H_{p}$ is a real selfadjoint operator, to get

$$
\sum_{j=1}^{2} \int_{p^{-1}(0)}\left(\left(H_{\bar{p}}+\bar{a}\right) H_{p} f_{j}\right) \bar{f}_{j} \lambda_{p, 0}(d \rho)=4 \int_{p^{-1}(0)}\left(\left(H_{\bar{p}}+\bar{a}\right) H_{p} f_{w}\right) \overline{f_{w}} \lambda_{p, 0}(d \rho),
$$

and

$$
\partial_{\bar{w}} \partial_{w} I=-2 \pi \int_{p^{-1}(0)}\left(\left(H_{\bar{p}}+\bar{a}\right) H_{p} f_{w}\right) \overline{f_{w}} \lambda_{p, 0}(d \rho)=2 \pi \int_{p^{-1}(0)} H_{p} f_{w} \overline{H_{p} f_{w}} \lambda_{p, 0}(d \rho) .
$$


Since $\left(H_{p}+a\right) H_{\bar{p}}$ is a real operator, the first equation in (8.29), can also be written

$$
\left(H_{\bar{p}}+\bar{a}\right)\left(H_{p} f_{w}-\frac{1}{2 i} \partial_{z} p\right)=0 .
$$

Here the orthogonal of the image of $H_{p}$ is the kernel of $-H_{p}^{*}=\left(H_{\bar{p}}+\bar{a}\right)$, so $(8.34)$ says that

$$
H_{p}\left(f_{w}\right)=\Pi\left(\frac{1}{2 i} \partial_{z} p\right)
$$

where $\Pi$ is the orthogonal projection onto the image of $H_{p}$. Then (8.33) becomes

$$
\partial_{\bar{w}} \partial_{w} I=\frac{\pi}{2} \int_{p^{-1}(0)}\left|\Pi \partial_{z} p\right|^{2} \lambda_{p, 0}(d \rho)
$$

Using this and (8.15) in (8.32), we finally get for $z=0$ :

$$
\partial_{\bar{z}} \partial_{z}\left(I\left(\Lambda_{z}, p_{z}\right)\right)=\frac{\pi}{2} \int_{p^{-1}(0)}\left|\partial_{z} p\right|^{2} \lambda_{p, 0}(d \rho)-\frac{\pi}{2} \int_{p^{-1}(0)}\left|\Pi \partial_{z} p\right|^{2} \lambda_{p, 0}(d \rho)
$$

which can also be written

$$
\partial_{\bar{z}} \partial_{z}\left(I\left(\Lambda_{z}, p_{z}\right)\right)=\frac{\pi}{2} \int_{p^{-1}(0)}\left|(I-\Pi) \partial_{z} p\right|^{2} \lambda_{p, 0}(d \rho)
$$

This formula gives some possible indication about the distribution of zeros of $\operatorname{det} p_{z}^{w}\left(x, h D_{x}\right)$.

9. The case when $p_{\Lambda_{0}}$ is of principal type. Let $\Lambda_{0}$ be an IR-manifold and $p$ holomorphic as in section 4 . Write $p_{0}=p_{\Lambda_{0}}$. Assume,

$$
p_{0}(\rho)=0 \Rightarrow d p_{0}(\rho) \neq 0 \text {. }
$$

Let $\rho_{0} \in p_{0}^{-1}(0)$. We can find local symplectic coordinates $(x, \xi)$ on neigh $\left(\rho_{0}, \Lambda_{0}\right)$, centered at $\rho_{0}$, such that with $x=\left(x^{\prime}, x_{n}\right), \xi=\left(\xi^{\prime}, \xi_{n}\right)$ :

$$
p_{0}=q(x, \xi)\left(\xi_{n}+i r\left(x, \xi^{\prime}\right)\right)
$$

with $q, r$ smooth, $q(0) \neq 0, r$ real valued with $r(0)=0$. We want to study

$$
\left\langle " d_{\Lambda} I\left(\Lambda_{0}, p\right) ", \delta \Lambda\right\rangle=\int\left\langle H_{\arg p_{0}}, d f\right\rangle \mu(d \rho)=-\int\left\langle d \arg p_{0}, H_{f}\right\rangle \mu(d \rho),
$$

with the integrals over $\Lambda_{0}$, where we recall that $d \arg p_{0}, H_{\arg p_{0}}$ extend from $\Lambda_{0} \backslash p_{0}^{-1}(0)$ to $L^{1}$ functions on $\Lambda_{0}$. Here $d f$ is the generator of the infinitesimal deformation $\delta \Lambda$ of $\Lambda_{0}$. We restrict the attention to $f \in C_{0}^{\infty}\left(\right.$ neigh $\left.\left(\rho_{0}, \Lambda_{0}\right) ; \mathbf{R}\right)$, so that $(9.2)$ is applicable with a non-vanishing $q$. Since

$$
\int\left\langle d \arg q, H_{f}\right\rangle \mu(d \rho)=0
$$


we get

$$
\left\langle " d_{\Lambda} I\left(\Lambda_{0}, p\right) ", \delta \Lambda\right\rangle=\int\left\langle H_{\arg p_{1}}, d f\right\rangle \mu(d \rho)=-\int\left\langle d \arg p_{1}, H_{f}\right\rangle \mu(d \rho),
$$

where $p_{1}=\xi_{n}+i r\left(x, \xi^{\prime}\right)$. We can view (9.4) as the limit when $\epsilon \rightarrow 0$ of

$$
\begin{aligned}
& \left\langle d_{\Lambda} J_{\epsilon}\left(\Lambda_{0}, p_{1}\right), \delta \Lambda\right\rangle=\operatorname{Re} i \int_{\Lambda_{0}} \frac{\overline{p_{1}}}{p_{1} \overline{p_{1}}+\epsilon^{2}} H_{f} p_{1} \mu(d \rho) \\
& =-\operatorname{Re} i \int_{\Lambda_{0}} \frac{\overline{p_{1}}}{p_{1} \overline{p_{1}}+\epsilon^{2}} H_{p_{1}} f \mu(d \rho)=\operatorname{Re} i \int_{\Lambda_{0}} H_{p_{1}}\left(\frac{\overline{p_{1}}}{p_{1} \overline{p_{1}}+\epsilon^{2}}\right) f \mu(d \rho) \\
& =\operatorname{Re} i \int_{\Lambda_{0}} \frac{\partial}{\partial \overline{p_{1}}}\left(\frac{\overline{p_{1}}}{p_{1} \overline{p_{1}}+\epsilon^{2}}\right) H_{p_{1}}\left(\overline{p_{1}}\right) f \mu(d \rho) .
\end{aligned}
$$

Since $r$ is independent of $\xi_{n}$, we see that

$$
H_{p_{1}} \overline{p_{1}}=H_{p_{1}}^{(n)} \overline{p_{1}}
$$

where $H_{p_{1}}^{(n)}$ denotes the $\left(\partial_{x_{n}}, \partial_{\xi_{n}}\right)$ component of $H_{p_{1}}$, i.e. the Hamilton field of $p_{1}$, in the variables $\left(x_{n}, \xi_{n}\right)$ with $\left(x^{\prime}, \xi^{\prime}\right)$ as parameters. We can insert this in the last expression of (9.5) and run the computation there backwards with $H_{p_{1}}^{(n)}, H_{f}^{(n)}$ instead of $H_{p_{1}}, H_{f}$, to get

$$
\left\langle d_{\Lambda} J_{\epsilon}\left(\Lambda_{0}, p\right), \delta \Lambda\right\rangle=\operatorname{Re} i \int \frac{\overline{p_{1}}}{p_{1} \overline{p_{1}}+\epsilon^{2}} H_{f}^{(n)} p_{1} \mu(d \rho) .
$$

Here we can integrate first in $\left(x_{n}, \xi_{n}\right)$ and then in $\left(x^{\prime}, \xi^{\prime}\right)$. The earlier discussion then applies to the $\left(x_{n}, \xi_{n}\right)$ integral, making use of the fact that $d^{(n)} \arg p_{1}:=d_{\left(x_{n}, \xi_{n}\right)} \arg p_{1}$ is uniformly in $L^{1}$, when $\left(x^{\prime}, \xi^{\prime}\right)$ varies. (Cf the remark on parameter dependence giving the last part of Proposition 4.4, where $t$ can be replaced by $\left(x^{\prime}, \xi^{\prime}\right)$.) We get

$$
\left\langle " d_{\Lambda} I\left(\Lambda_{0}, p\right) ", \delta \Lambda\right\rangle=\int\left\langle H_{\arg p_{1}}^{(n)}, d^{(n)} f\right\rangle \mu(d \rho)=-\int\left\langle d^{(n)} \arg p_{1}, H_{f}^{(n)}\right\rangle \mu(d \rho),
$$

where we recall that in view of the uniform boundedness of $d^{(n)} \arg p_{1}$ in $L^{1}$, we have

$$
\operatorname{Re} i \iint \frac{\overline{p_{1}}}{p_{1} \overline{p_{1}}+\epsilon^{2}} H_{f}^{(n)} p_{1} d x_{n} d \xi_{n} \rightarrow \iint\left\langle H_{\arg p_{1}}^{(n)}, d^{(n)} f\right\rangle d x_{n} d \xi_{n}, \epsilon \rightarrow 0,
$$

uniformly in $\left(x^{\prime}, \xi^{\prime}\right)$, since the LHS in (9.9) is equal to

$$
-\iint \frac{p_{1} \overline{p_{1}}}{p_{1} \overline{p_{1}}+\epsilon^{2}}\left\langle d^{(n)} \arg p_{1}, H_{f}^{(n)}\right\rangle d x_{n} d \xi_{n} .
$$

Now recall that the LHS of (9.9) is also equal to

$$
\begin{aligned}
& \operatorname{Re} i \iint \frac{\partial}{\partial \overline{p_{1}}}\left(\frac{\overline{p_{1}}}{p_{1} \overline{p_{1}}+\epsilon^{2}}\right)\left\{p_{1}, \overline{p_{1}}\right\} f d x_{n} d \xi_{n}= \\
& \operatorname{Re} \iint \frac{\epsilon^{2}}{\left(p_{1} \overline{p_{1}}+\epsilon^{2}\right)^{2}} i\left\{p_{1}, \overline{p_{1}}\right\} f d x_{n} d \xi_{n}=2 \iint \frac{\epsilon^{2}}{\left(\epsilon^{2}+\xi_{n}^{2}+r\left(x, \xi^{\prime}\right)^{2}\right)^{2}}\left(\partial_{x_{n}} r\right) f d x_{n} d \xi_{n} .
\end{aligned}
$$


As we already know in arbitrary dimension the limit only depends on the behaviour of $f$ near the points $\left(x_{n}, \xi_{n}\right)=\left(x_{n}, 0\right)$, with $r\left(x, \xi^{\prime}\right)=0$.

Assume for some fixed $\left(x^{\prime}, \xi^{\prime}\right)$, that

The zeros of $x_{n} \mapsto r\left(x, \xi^{\prime}\right)$ are all of finite order.

Assume that $x_{n}=0$ (say) is a zero of order $m$ and assume that $\operatorname{supp}_{x_{n}, \xi_{n}} f$ is contained in a small neighborhood of $(0,0)$. We have

$$
r=x_{n}^{m} u\left(x_{n}\right), u(0) \neq 0,
$$

and consider 4 different cases.

1) $m$ is odd and $u(0)>0$. Then $r$ considered as a function of $x_{n}$ is invertible near 0 and using that $\partial_{x_{n}} r d x_{n}=d r$, the integral in (9.11) becomes

$$
\iint \frac{\epsilon^{2}}{\left(\epsilon^{2}+\xi_{n}^{2}+y^{2}\right)^{2}} f\left(r^{-1}(y), \xi_{n}\right) d y d \xi_{n} \rightarrow \pi f(0,0) .
$$

2) $m$ is odd and $u(0)<0$. Now the map $r$ reverses the orientation and we get the limit $-\pi f(0,0)$.

3) $m$ is even and $u(0)>0$. The restrictions $r_{ \pm}$to a neighborhood of 0 on the positive and negative half axes respectively are now invertible and we can cut the integral into two, which simplifies to

$$
\begin{aligned}
\iint_{y \geq 0} \frac{\epsilon^{2}}{\left(\epsilon^{2}+\xi_{n}^{2}+y^{2}\right)^{2}}( & \left.f\left(r_{+}^{-1}(y), \xi_{n}\right)-f\left(r_{-}^{-1}(y), \xi_{n}\right)\right) d y d \xi_{n} \\
& \rightarrow \frac{\pi}{2}(f(0,0)-f(0,0))=0
\end{aligned}
$$

4) $m$ is even and $u(0)<0$. We get a change of sign compared to the previous case, and hence we still have the limit 0 .

Summing up, we get

Proposition 9.1.

(A) Assume for a given $\left(x^{\prime}, \xi^{\prime}\right)$, that (9.12) holds and define the index $\iota\left(x_{n}\right)$ of a zero $x_{n}$ of $r\left(x^{\prime}, \cdot, \xi^{\prime}\right)$ to be +1 if $r$ changes sign from - to,+-1 if $r$ changes sign from + to -, and to be 0 if we have no change of sign which happens precisely when $m$ is even. Then

$$
\iint \frac{\epsilon^{2}}{\left(\epsilon^{2}+\xi_{n}^{2}+r\left(x, \xi^{\prime}\right)^{2}\right)^{2}}\left(\partial_{x_{n}} r\right) f\left(x_{n}, \xi_{n}\right) d x_{n} d \xi_{n} \rightarrow \sum_{x_{n} ; r\left(x, \xi^{\prime}\right)=0} \pi \iota\left(x_{n}\right) f\left(x_{n}, 0\right) .
$$

(B) Assume that (9.12) holds for almost all $\left(x^{\prime}, \xi^{\prime}\right)$. Then

$$
\left\langle " d_{\Lambda} I\left(\Lambda_{0}, p\right) ", \delta \Lambda\right\rangle=2 \pi \iint\left(\sum_{x_{n} ; r\left(x, \xi^{\prime}\right)=0} \iota\left(x_{n}\right) f\left(x, \xi^{\prime}, 0\right)\right) d x^{\prime} d \xi^{\prime} .
$$

It is easy to construct an example of a $p_{0}$ of the form (9.2) which does not satisfy (9.12), such that $r$ has infinitely many zeros in $x_{n}$ and for which $\left\langle " d_{\Lambda} I\left(\Lambda_{0}, p\right) ", \delta \Lambda\right\rangle$ is a distribution of order 1 , but not a Radon measure, acting on $f$. 
The codimension 2 case, i.e. the case when $d p_{0}, d \overline{p_{0}}$ are linearly independent at every point of $p_{0}^{-1}(0)$, is equivalent to the case when $r\left(x, \xi^{\prime}\right)=0 \Rightarrow d r\left(x, \xi^{\prime}\right) \neq 0$, and in this case (9.12) does hold for almost all $\left(x^{\prime}, \xi^{\prime}\right)$. In this case we can get (9.16) more directly from (8.16). Indeed, consider the situation locally and assume that $p_{0}\left(\rho_{0}\right)=0$ and that

$$
d \operatorname{Re} p_{0}, d \operatorname{Im} p_{0} \text { are linearly independent at } \rho_{0} .
$$

Choose local symplectic coordinates $(x, \xi)$ centered at $\rho_{0}$, so that

$$
p_{0}=\xi_{n}+i r(x, \xi) .
$$

Notice that $r$ is now allowed to depend on $\xi_{n}$. Then by (9.17):

$$
d_{\left(x, \xi^{\prime}\right)} r(0) \neq 0 .
$$

Let us first assume that

$$
\left\{\operatorname{Re} p_{0}, \operatorname{Im} p_{0}\right\}\left(\rho_{0}\right) \neq 0,
$$

or equivalently that $\partial_{x_{n}} r(0) \neq 0$. Then near $\rho_{0}$, the surface $p_{0}^{-1}(0)$ is given by $\xi_{n}=0$, $r\left(x, \xi^{\prime}, 0\right)=0$ and can be parametrized by $x_{n}=x_{n}\left(x^{\prime}, \xi^{\prime}\right)$. The Liouville measure becomes

$$
L\left(d\left(x^{\prime}, \xi^{\prime}\right)\right)=\frac{d x^{\prime} d \xi^{\prime}}{\left|\partial_{x_{n}} r\right|}
$$

and hence (8.16) becomes

$$
\left\langle d_{\Lambda} I\left(\Lambda_{0}\right), \delta \Lambda\right\rangle=2 \pi \iint f\left(x^{\prime}, x_{n}\left(x^{\prime}, \xi^{\prime}\right), \xi^{\prime}, 0\right) \mu\left(d\left(x^{\prime}, \xi^{\prime}\right)\right),
$$

where

$$
\mu=\left\{\operatorname{Re} p_{0}, \operatorname{Im} p_{0}\right\} L\left(d\left(x^{\prime}, \xi^{\prime}\right)\right)=\frac{\partial_{x_{n}} r}{\left|\partial_{x_{n}} r\right|} d x^{\prime} d \xi^{\prime}=\operatorname{sgn}\left(\partial_{x_{n}} r\right) d x^{\prime} d \xi^{\prime} .
$$

Recall from Lemma 8.1, that this density is also given by

$$
\left.\frac{1}{(n-1) !} \sigma^{n-1}\right|_{p_{0}^{-1}(0)},
$$

with a suitable choice of orientation on $p_{0}^{-1}(0)$. Since $\xi_{n}=0$ on $p_{0}^{-1}(0)$, we see that even when (9.20) is not fulfilled (but still under the assumption (9.17)), the absolute value of the density appearing in (8.16) is bounded by

$$
\left|d x^{\prime} d \xi_{r^{-1}(0) \cap \xi_{n}^{-1}(0)}^{\prime}\right| .
$$

We already know that this density vanishes precisely when $\partial_{x_{n}} r=0$, and those points are precisely the ones where the projection

$$
r^{-1}(0) \cap \xi_{n}^{-1}(0) \ni\left(x, \xi^{\prime}\right) \mapsto\left(x^{\prime}, \xi^{\prime}\right) \in \mathbf{R}^{2(n-1)}
$$

is not a local diffeomorphism. The Lebesgue measure on $r^{-1}(0) \cap \xi_{n}^{-1}(0)$ is locally equivalent to the Liouville measure $L$, given by (9.21), and by (9.22), we have

$$
|\mu|=\left|\partial_{x_{n}} r\right| L
$$


We conclude that

$$
\int_{\left\{\left(x, \xi^{\prime}, 0\right) \in r^{-1}(0) \cap \operatorname{neigh}(0) ;\left|\partial_{x_{n}} r\right| \leq \epsilon\right\}}|\mu|\left(d\left(x, \xi^{\prime}\right)\right) \rightarrow 0, \epsilon \rightarrow 0,
$$

and this means that the points in $p_{0}^{-1}(0)$, where $\partial_{x_{n}} r\left(x, \xi^{\prime}, 0\right)=0$ can be neglected in the (local) formula for $\left\langle d_{\Lambda} I\left(\Lambda_{0}, p\right), \delta \Lambda\right\rangle$. Further, by Sard's theorem (here in an easy case), the set

$$
\left\{\left(x^{\prime}, \xi^{\prime}\right) \in \operatorname{neigh}\left(0, \mathbf{R}^{2(n-1)}\right) ; \exists x_{n} \in \operatorname{neigh}(0, \mathbf{R}), r\left(x, \xi^{\prime}, 0\right)=0, \partial_{x_{n}} r\left(x, \xi^{\prime}, 0\right)=0\right\}
$$

is of Lebesgue measure zero.

Summing up, under the assumption (9.17), and for $f \in C_{0}^{\infty}\left(\operatorname{neigh}\left(\rho_{0}, \Lambda_{0}\right)\right)$, we have, using the coordiates in (9.18):

$$
\left\langle d_{\Lambda} I\left(\Lambda_{0}, p\right), \delta \Lambda\right\rangle=2 \pi \iint d x^{\prime} d \xi^{\prime} \sum_{x_{n} ; r\left(x, \xi^{\prime}, 0\right)=0} \iota\left(x, \xi^{\prime}\right) f\left(x, \xi^{\prime}, 0\right)
$$

where $\iota\left(x, \xi^{\prime}\right)=\operatorname{sgn} \partial_{x_{n}} r\left(x, \xi^{\prime}, 0\right)$, if $\partial_{x_{n}} r\left(x, \xi^{\prime}, 0\right) \neq 0$ and $\iota\left(x, \xi^{\prime}\right)=0$ otherwise. Here we use the fact that the set $(9.26)$ is of measure 0 . Notice that if $\left(x^{\prime}, \xi^{\prime}\right)$ is not in that set, and we enumerate the zeros $x_{n}=x_{n}^{j}\left(x^{\prime}, \xi^{\prime}\right)$ of $r\left(x, \xi^{\prime}, 0\right)$ in increasing order for $j$ in a subinterval of $\mathbf{Z}$, then we may assume that $\operatorname{sgn} \partial_{x_{n}} r=(-1)^{j}$, and we get

$$
\begin{aligned}
\left|\sum_{j} \iota\left(x^{\prime}, x_{n}^{j}\left(x^{\prime}, \xi^{\prime}\right), \xi^{\prime}\right) f\left(x^{\prime}, x_{n}^{j}, \xi^{\prime}, 0\right)\right| & =\left|\sum_{k}\left(f\left(x^{\prime}, x_{n}^{2 k}, \xi^{\prime}, 0\right)-f\left(x^{\prime}, x_{n}^{2 k-1}, \xi^{\prime}, 0\right)\right)\right| \\
& \leq C \sup _{t}\left|\partial_{x_{n}} f\left(x^{\prime}, t, \xi^{\prime}, 0\right)\right|
\end{aligned}
$$

so we have a locally uniform bound on the RHS even when approaching the set (9.26) where the number of zeros may tend to infinity. (The need to use $|\nabla f|$ looks a little strange since we are working under assumptions that imply that our differential is a Radon measure with respect to $f$.)

We next want to globalize the formula (9.27) and make the global assumptions

$$
\begin{gathered}
p_{0}(\rho)=0 \Rightarrow d \operatorname{Re} p_{0}(\rho), d \operatorname{Im} p_{0}(\rho) \text { are independent, } \\
\operatorname{Re} p_{0}(\rho)=0 \Rightarrow d \operatorname{Re} p_{0}(\rho) \neq 0 .
\end{gathered}
$$

If we choose local symplectic coordinates such that $\operatorname{Re} p_{0}=\xi_{n}$, then the Liouville measure on $\left(\operatorname{Re} p_{0}\right)^{-1}(0)$ is $d x^{\prime} d \xi^{\prime} d t$ where $t=x_{n}$ corresponds to the time of the Hamilton flow of Re $p_{0}$. A contribution from a zero in $(9.27): \iota\left(x^{\prime}, x_{n}^{j}, \xi^{\prime}\right) f\left(x^{\prime}, x_{n}^{j}, \xi^{\prime}, 0\right)$ can be expressed as

$$
2 \pi \iiint_{t \in I_{x^{\prime}, \xi^{\prime}}} \frac{1}{T\left(x^{\prime}, \xi^{\prime}\right)} \iota\left(x^{\prime}, x_{n}^{j}, \xi^{\prime}\right) f\left(x^{\prime}, x_{n}^{j}, \xi^{\prime}, 0\right) d t d x^{\prime} d \xi^{\prime},
$$

where $I_{x^{\prime}, \xi^{\prime}}$ is some interval of length $T\left(x^{\prime}, \xi^{\prime}\right)>0$.

We shall use this without refering to $(9.18)$ first in the case when the length of the time interval is fixed and then for a special choice of variable length. First in the case of a fixed length we have

$$
\begin{aligned}
& \left\langle d_{\Lambda} I\left(\Lambda_{0}, p\right), \delta \Lambda\right\rangle \\
& =\frac{2 \pi}{2 T} \int_{\left(\operatorname{Re} p_{0}\right)^{-1}(0)} \sum_{t \in]-T, T\left[; \operatorname{Im} p_{0}\left(\Phi_{t}(\rho)\right)=0\right.} \iota\left(\Phi_{t}(\rho)\right) f\left(\Phi_{t}(\rho)\right) \lambda_{\operatorname{Re} p_{0}=0}(d \rho),
\end{aligned}
$$


where $\Phi_{t}(\rho)=\exp t H_{\operatorname{Re} p_{0}}(\rho), \lambda_{\operatorname{Re} p_{0}=0}$ is the Liouville measure on $\left(\operatorname{Re} p_{0}\right)^{-1}(0)$ and $\iota(\rho)$ is equal to \pm 1 if $\pm \operatorname{Im} p_{0}\left(\Phi_{s}(\rho)\right)$ has a simple zero at $s=0$ with a change of sign from - to + and is 0 otherwise. Notice that for $\rho \in\left(\operatorname{Re} p_{0}\right)^{-1}(0)$ away from some set of measure 0 , all the zeros of $\mathbf{R} \ni t \mapsto \operatorname{Im} p_{0}\left(\Phi_{t}(\rho)\right)$ are simple.

Lemma 9.2. The set of points $\rho \in\left(\operatorname{Re} p_{0}\right)^{-1}(0)$, such that $\operatorname{Im} p_{0}\left(\Phi_{t}(\rho)\right)$ is $<0$ for some $t \in \mathbf{R}$ and $\geq 0$ for all sufficiently large positive $t$, is of measure 0 . Let

Proof. Let $\Omega$ be the set in question, so that $\Omega$ is a union of non-closed trajectories.

$$
\Omega_{+}=\left\{\rho \in \Omega ; \operatorname{Im} p_{0}\left(\Phi_{t}(\rho)\right) \geq 0, \text { for } t \geq 0\right\} .
$$

Then

$$
\Phi_{t}\left(\Omega_{+}\right) \rightarrow\left\{\begin{array}{l}
\emptyset, t \rightarrow+\infty \\
\Omega, t \rightarrow-\infty
\end{array}\right.
$$

so by the dominated convergence theorem,

$$
\lambda_{\operatorname{Re} p_{0}=0}\left(\Phi_{t}\left(\Omega_{+}\right)\right) \rightarrow\left\{\begin{array}{l}
0, t \rightarrow+\infty \\
\lambda_{\operatorname{Re} p_{0}=0}(\Omega), t \rightarrow-\infty .
\end{array}\right.
$$

But $\Phi_{t}$ is measure preserving so $\lambda_{\operatorname{Re} p_{0}=0}\left(\Phi_{t}\left(\Omega_{+}\right)\right)$is independent of $t$ and we conclude that $\lambda_{\operatorname{Re} p_{0}=0}(\Omega)=0$.

We have 4 variants of the lemma since we may replace $\operatorname{Im} p_{0}$ by $-\operatorname{Im} p_{0}$ and $t$ by $-t$. It follows that for $\rho$ outside some set of Liouville measure 0 , we are in one of the following three cases:

1) $\operatorname{Im} p_{0}\left(\Phi_{t}(\rho)\right) \geq 0$ for all $t \in \mathbf{R}$,

2) $\operatorname{Im} p_{0}\left(\Phi_{t}(\rho)\right) \leq 0$ for all $t \in \mathbf{R}$,

3) $t \mapsto \operatorname{Im} p_{0}\left(\Phi_{t}(\rho)\right)$ has infinitely many changes of sign both when $t \rightarrow+\infty$ and when $t \rightarrow-\infty$. Moreover each zero is simple.

Here the last sentence in 3) could be added because of the observation prior to Lemma 9.2.

Define a function $F$ on $\left(\operatorname{Re} p_{0}\right)^{-1}(0)$ in the following way: Put $F(\rho)=0$ in the cases 1) or 2) above or if $t \mapsto \operatorname{Im} p_{0}\left(\Phi_{t}(\rho)\right)$ has at least one zero which is not simple.

In case 3) let $t(\rho) \leq 0<s(\rho)$ be the zeros of $\operatorname{Im} p_{0}\left(\Phi_{t}(\rho)\right)$ which are closest to 0 in the sense that $\operatorname{Im} p_{0}\left(\Phi_{t}(\rho)\right) \neq 0$ for $t(\rho)<t<s(\rho)$. If $\operatorname{Im} p_{0}(\Phi \cdot(\rho))_{\mid] t(\rho), s(\rho)[}>0$ we put $F(\rho)=0$ and in the opposite case we put

$$
\begin{aligned}
& F(\rho)=\frac{1}{s(\rho)-t(\rho)}\left(f\left(\Phi_{s(\rho)}(\rho)\right)-f\left(\Phi_{t(\rho)}(\rho)\right)\right) \\
& =\frac{1}{s(\rho)-t(\rho)} \int_{t(\rho)}^{s(\rho)}\left(H_{\operatorname{Re} p_{0}} f\right)\left(\Phi_{t}(\rho)\right) d t .
\end{aligned}
$$

Alternatively we could take

$$
\widetilde{F}(\rho)=H_{\operatorname{Re} p_{0}}(f)(\rho)
$$

in the last case and $\widetilde{F}(\rho)=F(\rho)=0$ in the other cases.

With this choice of $F$ or with $F$ replaced by $\widetilde{F}$, we have

$$
\left\langle d_{\Lambda} I\left(\Lambda_{0}, p\right), \delta \Lambda\right\rangle=2 \pi \int_{\left(\operatorname{Re} p_{0}\right)^{-1}(0)} F(\rho) \lambda_{\operatorname{Re} p_{0}=0}(d \rho) .
$$


Before continuing the main discussion, we need an auxiliary result:

LEMMA 9.3. Let $M$ be a compact smooth manifold and let $v$ be a smooth nonvanishing vector field on $M$. If $f_{0} \in C^{\infty}(M ; \mathbf{R})$, then we can find $f \in C^{\infty}(M ; \mathbf{R})$ arbitrarily close to $f_{0}$ in the $C^{\infty}$ topology, such that

$$
v(f)(x)=0 \Rightarrow d(v(f))(x) \neq 0,
$$

for every $x \in M$.

Proof. We can cover $M$ by finitely many open sets $\Omega_{j}, j=1, . ., N$ such that for each $j$ there is a real function $g_{j} \in C^{\infty}(M)$ with $v g_{j}=1$ in $\bar{\Omega}_{j}$. Consider $f_{1}=f_{0}-\epsilon_{1} g_{1}$. In $\bar{\Omega}_{1}$ we have $v\left(f_{1}\right)=v\left(f_{0}\right)-\epsilon_{1}$, and we can choose $\epsilon_{1}$ arbitrarily small, so that $\epsilon_{1}$ is not a critical value of $v\left(f_{0}\right)$ on $\bar{\Omega}_{1}$. Then there exists $\delta_{1}>0$, such that

$$
\left|\nabla\left(v f_{1}\right)\right|+\left|v f_{1}\right| \geq \delta_{1}
$$

in $\bar{\Omega}_{1}$. Define $f_{2}=f_{1}-\epsilon_{2} g_{2}$, so that $v\left(f_{2}\right)=v\left(f_{1}\right)-\epsilon_{2}$ in $\bar{\Omega}_{2}$. We choose $\epsilon_{2}$ very small and not equal to any critical value of $v\left(f_{1}\right)$ on $\Omega_{2}$. Then (9.35) holds in $\bar{\Omega}_{1}$ after replacing $\left(f_{1}, \delta_{1}\right)$ by $\left(f_{2}, \delta_{1} / 2\right)$ and there is some $\delta_{2}>0$ such that

$$
\left|\nabla\left(v f_{2}\right)\right|+\left|v f_{2}\right| \geq \delta_{2}
$$

on $\overline{\Omega_{1} \cup \Omega_{2}}$. Continuing this procedure we get the Lemma after $N$ steps.

We next consider the case when $p_{0}$ is of real principal type. Let $\Lambda_{0}$ be an IR manifold and $p$ a holomorphic function as in section 4 . Let $p_{0}=p_{\Lambda_{0}}$. We now assume

$$
\begin{aligned}
& p_{0} \text { is real-valued. } \\
& d p_{0} \neq 0 \text { on } p_{0}^{-1}(0),
\end{aligned}
$$

and that $p_{0}^{-1}(0) \neq \emptyset$. Let neigh $(0, \mathbf{R}) \ni t \mapsto \Lambda_{t}$ be a smooth deformation of IR manifolds as in section 4 , with $\Lambda_{t=0}=\Lambda_{0}$. Let $f_{t} \in C_{b}^{\infty}\left(\Lambda_{t} ; \mathbf{R}\right)$ be a corresponding smooth family of generators. Then (7.3), (7.4) are satisfied, so Theorem 7.1 applies and shows that

$$
I\left(\Lambda_{t}, p\right) \geq I\left(\Lambda_{0}, p\right)-C_{N}|t|^{N}
$$

for every $N \in \mathbf{N}$. $\Lambda_{0}$ is therefore a critical manifold in a generalized sense and we shall now see that the derivative of $t \mapsto I\left(\Lambda_{t}, p\right)$ has a jump discontinuity at $t=0$ if $f_{0}$ is appropriately chosen.

Apply Lemma 9.3 with $M=p_{0}^{-1}(0), v=H_{p_{0}}$ and conclude that there exists $f=f_{0} \in C_{b}^{\infty}\left(\Lambda_{0} ; \mathbf{R}\right)$ such that:

$$
d H_{p_{0}} f_{\left.\right|_{M}} \neq 0 \text { whereever } H_{p_{0}} f=0 \text {. }
$$

From now on we will assume that $f_{0}$ has this property.

Identifying $\Lambda_{t}$ and $\Lambda_{0}$ by means of the symplectic map $\kappa_{0, t}: \Lambda_{0} \rightarrow \Lambda_{t}$ of section 1 , we can view $p_{t}$ as a function on $\Lambda_{0}$. By Taylor expansion, we get

$$
p_{t}(\rho)=p_{0}(\rho)+i t H_{f} p_{0}(\rho)+\mathcal{O}\left(t^{2}\right) \text { in } C_{b}^{\infty} .
$$


Hence

$$
\operatorname{Re} p_{t}=p_{0}+\mathcal{O}\left(t^{2}\right), \frac{1}{t} \operatorname{Im} p_{t}=-H_{p_{0}} f+\mathcal{O}(t) .
$$

For $t \neq 0$, we put

$$
\Sigma_{t}=\left\{\rho \in \Lambda_{0} ; p_{t}(\rho)=0\right\}
$$

and for $t=0$ :

$$
\Sigma_{0}=\left\{\rho \in \Lambda_{0} ; p_{0}(\rho)=0, H_{p_{0}} f(\rho)=0\right\} .
$$

From $(9.40,42)$ it follows that $\Sigma_{t}, \Sigma_{0}$ are smooth compact submanifolds of $\Lambda_{0}$ of codimension 2 , and that $\operatorname{dist}\left(\Sigma_{0}, \Sigma_{t}\right)=\mathcal{O}(t)$, in the natural sense. We notice that $\Sigma_{t}=\widetilde{p}_{t}^{-1}(0)$, where $\widetilde{p}_{t}:=\operatorname{Re} p_{t}+i \frac{\operatorname{Im} p_{t}}{t}$, for $t \neq 0$ and $\widetilde{p}_{0}=p_{0}-i H_{p_{0}} f$. Then $\widetilde{p}_{t}-\widetilde{p}_{0}=\mathcal{O}(t)$ in $C_{b}^{\infty}$ and $d \operatorname{Re} \widetilde{p}_{t}, d \operatorname{Im} \widetilde{p}_{t}$ are linearly independent on $\Sigma_{t}$. For $t \neq 0$, we have

$$
\frac{i}{2}\left\{p_{t}, \overline{p_{t}}\right\} \lambda_{p_{t}=0}=(\operatorname{sgn} t)\left\{\widetilde{p}_{t}, \overline{\tilde{p}_{t}}\right\} \lambda_{\tilde{p}_{t}=0}
$$

on $\Sigma_{t}$. Further

$$
\frac{i}{2}\left\{\widetilde{p}_{t}, \widetilde{p}_{t}\right\}=\left\{\operatorname{Re} \widetilde{p}_{t}, \operatorname{Im} \widetilde{p}_{t}\right\}=\left\{\operatorname{Re} \widetilde{p}_{0}, \operatorname{Im} \widetilde{p}_{0}\right\}+\mathcal{O}(t)=-H_{p_{0}}^{2} f+\mathcal{O}(t)
$$

and we get for $t \neq 0$ (cf. $(8.16))$ :

$$
\begin{aligned}
\partial_{t} I\left(\Lambda_{t}, p\right)= & (\operatorname{sgn} t) 2 \pi \int_{\Sigma_{t}} f_{t} \frac{i}{2}\left\{\widetilde{p}_{t}, \overline{\widetilde{p}_{t}}\right\} \lambda_{\tilde{p}_{t}=0}(d \rho) \\
& =(\operatorname{sgn} t) 2 \pi \int_{\Sigma_{0}} f_{0} \frac{i}{2}\left\{\widetilde{p}_{0}, \overline{\widetilde{p}_{0}}\right\} \lambda_{\tilde{p}_{0}=0}(d \rho)+\mathcal{O}(t) .
\end{aligned}
$$

From this and (9.39) we see that

$$
2 \pi \int_{\Sigma_{0}} f_{0} \frac{i}{2}\left\{\widetilde{p}_{0}, \overline{\widetilde{p}_{0}}\right\} \lambda_{\tilde{p}_{0}=0}(d \rho) \geq 0 .
$$

The following proposition shows that most of the time we have strict inequality and hence that $\partial_{t} I\left(\Lambda_{t}, p\right)$ has a jump discontinuity at $t=0$. The proof also gives a more direct explanation of (9.47).

Proposition 9.4. Assume that there is a point $\rho_{0} \in \Sigma_{0}$ where $\frac{i}{2}\left\{\widetilde{p}_{0}, \overline{\widetilde{p}_{0}}\right\}=$ $-H_{p_{0}}^{2} f \neq 0$. Then we have strict inequality in (9.47).

Proof. The expression (9.47) if formally equal to $\left\langle d_{\Lambda} I\left(\Lambda_{0}, \widetilde{p}_{0}\right), \delta \Lambda\right\rangle$, with $\delta \Lambda$ generated by $f_{0}$. (This is only formal, since $\widetilde{p}_{0}$ does not in general have a holomorphic extension.) The discussion starting at (9.17) applies and we have (9.33) with $F$ defined there with $f$ equal to $f_{0}$ and with $p_{0}$ replaced by $\widetilde{p}_{0}$. The points $\rho \in p_{0}^{-1}(0)$ of type $3)$ are the ones for which $t \mapsto-\left(H_{p_{0}} f\right)\left(\Phi_{t}(\rho)\right)$ has only simple zeros, infinitely many near both $t=+\infty$ and $t=-\infty$. The assumption in the proposition implies (and is in fact equivalent to) the fact that the points of type 3 ) form a set of measure $>0$. Let $\rho$ be point of type 3) for which $F(\rho)$ maybe $\neq 0$ i.e. for which the closest zeros $t(\rho) \leq 0<s(\rho)$ of $\operatorname{Im} \widetilde{p}_{0}\left(\Phi_{t}(\rho)\right)=-\left(H_{p_{0}} f\right)\left(\Phi_{t}(\rho)\right)$ are such that $-\left(H_{p_{0}}^{2} f\right)\left(\Phi_{t}(\rho)\right)$ is $<0$ for $t=t(\rho)$ and $>0$ for $t=s(\rho)$. Then $t=t(\rho), s(\rho)$ are subsequent local extrema 
of the function $t \mapsto f\left(\Phi_{t}(\rho)\right)$ which is strictly increasing between the two points. Consequently $f\left(\Phi_{s(\rho)}(\rho)\right)-f\left(\Phi_{t(\rho)}(\rho)\right)>0$. We conclude that $F(\rho)$ in $(9.31)$ is $\geq 0$ with strict inequality on a set of measure $>0$. As already noticed,

$$
2 \pi \int_{\Sigma_{0}} f_{0} \frac{i}{2}\left\{\widetilde{p}_{0}, \overline{\tilde{p}_{0}}\right\} \lambda_{\tilde{p}_{0}=0}(d \rho)=2 \pi \int_{p_{0}^{-1}(0)} F(\rho) \lambda_{p_{0}=0}(d \rho) .
$$

and the proposition follows.

At least in the case when $f=f_{0}$ extends to a bounded holomorphic function in a tube and $\Lambda_{t}=\exp i H_{f}\left(\Lambda_{0}\right)$, there is a more general way of detecting a jump discontinuity at $t=0$ of $\partial_{t} I\left(\Lambda_{t}, p\right)$, when $p_{0}^{-1}(0)$ contains a real hypersurface, even without assuming that $p_{0}$ is of principal type. This can be done by examining the second derivative and the Levi form with respect to $t$, and we hope to develop this point of view in some future work. Another possible approach would be to look for jump discontinuities in $d \arg p_{t}$ as a function of $t$.

10. Examples. We consider two simple examples with $\Lambda$ fixed and with $p$ depending on a complex spectral parameter. Let $\Lambda_{0}$ and $p$ be as in section 4 , and assume that $p=p(z)$ depends holomorphically on $z \in \Omega$ where $\Omega \subset \subset \mathbf{C}$ is open. Recall that

$$
I\left(\Lambda_{0}, p(z)\right)=\frac{1}{2} \int_{\Lambda_{0}} \log (p(z) \overline{p(z)}) \mu(d \rho) .
$$

Using (8.3), we get

$$
\left.\partial_{z} \partial_{\bar{z}}\left(\frac{1}{2} \log (p(\rho, z) \overline{p(\rho, z)})\right)=\pi \delta(p(\rho, z)) \partial_{z} p(\rho, z)\right) \overline{\partial_{z} p(\rho, z)}
$$

near simple zeros of $z \mapsto p(\rho, z)$. We want to discuss the special case when $p(\rho, z)=$ $p(\rho)-z$, with $p(\rho)$ as in section 4 , but since this symbol tends to $1-z$ rather than 1 when $\rho \rightarrow \infty$, we need to consider a modified symbol, corresponding to the estimate of a relative determinant. Let $\widetilde{p}(\rho) \in S(\Lambda, 1)$ with

$$
p(\rho)-\widetilde{p}(\rho)=\mathcal{O}\left(\langle\rho\rangle^{m}\right), m<-2 n .
$$

Assume that $\Omega \subset \subset \mathbf{C} \backslash\{1\}$ and that

$$
\widetilde{p}(\rho) \notin \bar{\Omega}, \rho \in \Lambda_{0} .
$$

Consider

$$
I(z)=I\left(\Lambda_{0}, \frac{p-z}{\widetilde{p}-z}\right)=\frac{1}{2} \int_{\Lambda_{0}}(\log ((p-z) \overline{(p-z)}-\log ((\widetilde{p}-z) \overline{(\widetilde{p}-z)})) \mu(d \rho),
$$

for $z \in \Omega$. Then,

$$
\partial_{z} \partial_{\bar{z}} I(z)=\pi \int_{\Lambda_{0}} \delta(p(\rho)-z) \mu(d \rho)
$$

This means that if $\phi \in C_{0}^{\infty}(\Omega)$, then

$$
\int_{\mathbf{C}} \phi(z)\left(\partial_{z} \partial_{\bar{z}} I(z)\right) L(d z)=\pi \int_{\Lambda_{0}} \phi(p(\rho)) \mu(d \rho) .
$$


In other words $\partial_{z} \partial_{\bar{z}} I(z)$ can be described in $\Omega$ as the direct image under $p$ of the symplectic volume on $\Lambda_{0}$ :

$$
\partial_{z} \partial_{\bar{z}} I(z)=\pi p_{*}(\mu)
$$

If we assume that $p_{0}=p_{\Lambda_{0}}$ is real-valued and that $\left.\Omega \cap \mathbf{R} \subset\right]-\infty, 0[$, then the measure $\partial_{z} \partial_{\bar{z}} I(z)$ on $\Omega$ is supported in $\Omega \cap \mathbf{R}$, and is given there by the Stieltjes measure $\pi d V(E)$, with

$$
V(E)=\int_{\rho ; p(\rho) \leq E} \mu(d \rho) .
$$

For our second example, we drop the assumption that $p_{0}=p_{\Lambda_{0}}$ be real and assume instead that $\Omega$ is a small neighborhood of 0 in $\mathbf{C}$, that $p_{0}^{-1}(0)$ consists of precisely one point $\rho_{0}$ and that $\left|p_{0}(\rho)\right| \sim \operatorname{dist}\left(\rho, \rho_{0}\right)^{2}$, for $\rho \in \operatorname{neigh}\left(\rho_{0}, \Lambda_{0}\right)$. Let $\nu=p_{0 *}(\mu)$, so that $\partial_{z} \partial_{\bar{z}} I(z)=\pi \nu$. We observe that $\nu(D(0, r)) \sim r^{n}$.

Let $\widehat{p}$ be a second holomorphic function with the same properties and the same point $\rho_{0}$, and assume that

$$
\widehat{p}_{0}(\rho)-p_{0}(\rho)=\mathcal{O}\left(\operatorname{dist}\left(\rho, \rho_{0}\right)^{2 N_{0}}\right)
$$

for some $N_{0}>1$. (Actually the discussion is valid with a comparison function $\widehat{p}_{0} \in$ $C_{b}^{\infty}\left(\Lambda_{0}\right)$ which does not necessarily have a holomorphic extension $\widehat{p}$.) Let $V=V_{r} \subset$ $\mathbf{C} \backslash\{0\}$ with $V \subset D(0, r), 0<r \leq 1$. If $z=p_{0}(\rho) \in V$ for some $\rho \in \Lambda_{0}$, then $\operatorname{dist}\left(\rho, \rho_{0}\right)=\mathcal{O}\left(r^{1 / 2}\right)$ and it follows that $\widehat{p}_{0}(\rho) \in V+D\left(0, C r^{N_{0}}\right)$. Consequently,

$$
\nu(V) \leq \widehat{\nu}\left(V+D\left(0, C r^{N_{0}}\right)\right),
$$

where $\widehat{\nu}=\widehat{p}_{0 *}(\mu)$. The preceding argument is symmetric in $p, \widehat{p}$, so we also have

$$
\widehat{\nu}(V) \leq \nu\left(V+D\left(0, C r^{N_{0}}\right)\right) .
$$

The last two estimates express that $\partial_{z} \partial_{\bar{z}} I(z)$ and $\partial_{z} \partial_{\bar{z}} \widehat{I}(z)$ (where $\widehat{I}(z)$ is defined as in (10.4), with $\widehat{p}$ replacing $p$ ) are close to each other near $z=0$.

The last example is motivated by the study of resonances for the semi-classical Schrödinger operator that are generated by a non-degenerate critical point of the potential, and we refer to $[\mathrm{HeSj}],[\mathrm{Sj} 3],[\mathrm{BrCoDu}]$ for more details. After a suitable complex scaling or application of the theory of $[\mathrm{HeSj}]$, we can reduce the study of resonances of a Schrödinger operator $-h^{2} \Delta+V(x)$ in $\mathbf{R}^{n}$ in a neighborhood of 0 (say), to that of the zeros of a relative determinant $\operatorname{det}(P-z)(\widetilde{P}-z)^{-1}$, where $P$ is a realization of $-h^{2} \Delta+V$ in a suitable space that is associated to an IR-manifold $\Lambda_{0}$ and with principal symbol $p$, and $\widetilde{P}$ is a Schrödinger operator with a potential $\widetilde{V}$ such that $\widetilde{V}-V$ is sufficiently short range and such that $\widetilde{P}$ has no resonances in some neighborhood of 0 . (Actually, the property $p(\rho) \rightarrow 1, \rho \rightarrow \infty$, must be replaced by a more general ellipticity property, but that does not affect the validity of the discussion above.) Then as we saw in section 3 , though we now have to appeal to the [HeSj] theory which is similar but slightly heavier than the one we explained in that section, we obtain

$$
\log \left|\operatorname{det}\left((P-z)(\widetilde{P}-z)^{-1}\right)\right| \leq(2 \pi h)^{-n}(I(z)+o(1)), h \rightarrow 0 .
$$

The symbol $\widehat{p}$ may be obtained by using a quadratic approximation of $V$ at the critical point and then we can take $N_{0}=3 / 2$, or it can be obtained by using a (more elaborate) 
Birkhoff normal form in which case we may have larger values of $N_{0}$ sometimes even $N_{0}=+\infty$.

As a special case, we may take $n=2$, and assume that for some local smooth symplectic coordinates $(x, \xi)$, centered at $\rho_{0}$, we have

$$
\widehat{p}_{0}(\rho)=f\left(\frac{1}{2}\left(\xi_{1}^{2}+x_{1}^{2}\right), \frac{1}{2}\left(\xi_{2}^{2}+x_{2}^{2}\right)\right)
$$

near $\rho_{0}$ with $f \in C^{\infty}($ neigh $(0, \mathbf{C}))$ with

$$
f\left(\iota_{1}, \iota_{2}\right)=\mu_{1} \iota_{1}-i \mu_{2} \iota_{2}+\mathcal{O}\left(\iota^{2}\right)
$$

with $\mu_{1}, \mu_{2}>0$. Then near $0: \widehat{\nu}=2 \pi f_{*} L$, where $L$ is the Lebesgue measure on the 4th quadrant in $\mathbf{R}^{2}$.

\section{REFERENCES}

[AgCo] J. Aguilar, J.M. Combes, A class of analytic perturbations for one-body Schrödinger Hamiltonians, Comm. Math. Phys., 22(1971), pp. 269-279.

[BaCo] E. BAlslev, J.M. Combes, Spectral properties of many-body Schrödinger operators with dilatation-analytic interactions, Comm. Math. Phys., 22(1971), pp. 280-294.

[BoSj] L. Boutet de Monvel, J. Sjöstrand, Sur la singularité des noyaux de Bergman et de Szegö, Journées équations aux Dérivées Partielles de Rennes (1975), pp. 123-164. Astérisque, No. 34-35, Soc. Math. France, Paris, 1976.

[BrCoDu] P. Briet, J.M. Combes, P. Duclos, On the location of resonances for Schrödinger operators in the semiclassical limit. I. Resonance free domains, J. Math. Anal. Appl., 126(1)(1987), pp. 90-99.

[Bu] N. BuRQ, Décroissance de l'énergie locale de l'équation des ondes pour le problème extérieur et absence de résonances au voisinage du réel, Acta Math., 180(1998), pp. 1-29.

[Da] E.B. Davies, Semi-classical states for non-self-adjoint Schrödinger operators, Comm. Math. Phys., 200(1999), pp. 35-41.

[DiSj] M. Dimassi, J. SJöstrand, Spectral asymptotics in the semi-classical limit, London Math. Soc. Lecture Notes Series 269, Cambridge University Press 1999.

[GoKr] I.C. Gohberg, M.G. Krĕn, Introduction to the theory of linear nonselfadjoint operators, Translations of Mathematical Monographs, Vol. 18 American Mathematical Society, Providence, R.I. 1969.

[GuZw] L. Gulllopé, M. Zworski, Scattering asymptotics for Riemann surfaces, Ann. of Math., 145 (1997), no. 3, pp. 597-660.

[HeSj] B. Helffer, J. Suöstrand, Résonances en limite semi-classique, Mém. Soc. Math. France (N.S.) No. 24-25, (1986).

[Hö1] L. HöRmANDER, On the singularities of partial differential equations, 1970 Proc. Internat. Conf. on Functional Analysis and Related Topics (Tokyo, 1969) pp. 31-40 Univ. of Tokyo Press, Tokyo.

[Hö2] L. HÖRMANDER, On the existence and the regularity of solutions of linear pseudo-differential equations, Enseignement Math., (2) 17 (1971), pp. 99-163.

[HöWe] L. Hörmander, J. Wermer, Uniform approximation on compact sets in $\mathbf{C}^{n}$, Math. Scand., 23(1968), pp. 5-21.

[LeRo] G. Lebeau, L. Robbiano, Contrôle exact de l'équation de la chaleur, Comm. PDE, 20(1995), pp. 335-356.

[Ma] B. Malgrange, Ideals of differentiable functions, Tata Institute of Fundamental Research Studies in Mathematics, No. 3 Tata Institute of Fundamental Research, Bombay; Oxford University Press, London 1967. 
[MaMa] A.S. Markus, V.I. Matsaev, Comparison theorems for spectra of linear operators, and spectral asymptotics, Trans. Moscow. Math. Soc., 1984(1), pp. 139-187, and Trudy Moskov. Matem. O. 45(1984).

[Me] R. Melrose, Polynomial bound on the distribution of poles in scattering by an obstacle, Proc. Journées e.d.p. St Jean de Monts, 1984, Soc. Math. de France.

[MeSj] A. Melin, J. SJöstrand, Fourier integral operators with complex valued phase functions, Springer Lecture Notes in Math., no 459.

[Sj1] J. SJöstrand, Singularités analytiques microlocales, Astérisque, 95(1982).

[Sj2] J. SJöstRAND, Function spaces associated to global I-Lagrangian manifolds, pp. 369-423 in Structure of solutions of differential equations, Katata/Kyoto, 1995, World Scientific 1996.

[Sj3] J. SJöstRAnd, Semiclassical resonances generated by a non-degenerate critical point, Springer LNM, 1256, pp. 402-429.

[Sj4] J. Suöstrand, A traceformula and review of some estimates for resonances, pp. 377-437 in Microlocal Analysis and Spectral Theory, NATO ASI Series C, vol.490, Kluwer 1997.

[Sj5] J. SuÖSTRAnd, Geometric bounds on the density of resonances for semiclassical problems, Duke Math. J., 60(1)(1990), pp. 1-57.

[SjZw] J. Suöstrand, M. Zworski, Complex scaling and the distribution of scattering poles, Journal of the AMS, 4(4)(1991), pp. 729-769.

[Vo] G. Vodev, Sharp bounds on the number of scattering poles for perturbations of the Laplacian, Comm. Math. Phys., 146(1)(1992), pp. 205-216.

[Ze] M. Zerzeri, Majoration du nombre de résonances près de l'axe réel pour une perturbation, à support compact, abstraite, du laplacien, preprint, Université de Paris Nord, 1999, Comm. PDE, to appear.

[Zw1] M. Zworski, Poisson formulae for resonances, Sém. équations aux Dérivées Partielles, 1996-1997, Exp. No. XIII, Ecole Polytech., Palaiseau, 1997.

[Zw2] M. Zworski, A remark on a paper by E.B. Davies, Proc. AMS, to appear.

[Zw3] M. Zworski, Sharp polynomial bounds on the number of scattering poles, Duke Math. J. 59(2)(1989), pp. 311-323 
A. MELIN AND J. SJÖSTRAND 